\title{
PROTEOMIC ANALYSIS OF THE CRUSTACEAN MOLTING GLAND (Y-ORGAN) OVER THE COURSE OF THE MOLT CYCLE
}

\author{
A Thesis \\ presented to \\ the Faculty of California Polytechnic State University, \\ San Luis Obispo
}

\author{
In Partial Fulfillment \\ of the Requirements for the Degree \\ Master of Science in Biological Sciences
}

\author{
by \\ Talia Brianne Head \\ August 2017
}


(C) 2017

Talia Brianne Head ALL RIGHTS RESERVED 
TITLE:

Proteomic Analysis of the Crustacean Molting Gland (Y-organ) Over the Course of the Molt Cycle
AUTHOR:
Talia Brianne Head

DATE SUBMITTED:

August 2017

COMMITTEE CHAIR: Lars Tomanek, Ph.D.

Professor of Biology

California Polytechnic State University

COMMITTEE MEMBER: $\quad$ Donald Mykles, Ph.D.

Professor of Biology

Colorado State University

COMMITTEE MEMBER: Kristin Hardy, Ph.D.

Associate Professor of Biology

California Polytechnic State University 


\author{
ABSTRACT \\ Proteomic Analysis of the Crustacean Molting Gland (Y-organ) Over the \\ Course of the Molt Cycle \\ Talia Brianne Head
}

Molting in crustaceans is a highly complex physiological process involving negative regulation by two paired endocrine glands, the $\mathrm{X}$-organ/sinus gland complex $(\mathrm{XO} / \mathrm{SG})$ and the $\mathrm{Y}$-organ (YO). The $\mathrm{XO} / \mathrm{SG}$ complex is responsible for making molt-inhibiting hormone (MIH) which negatively regulates synthesis of the molting hormones, ecdysteroids, by the YO. Analysis of gene expression in the XOs and YOs has led to the development of a proposed molecular signaling pathway which regulates ecdysteroidogenesis and subsequent molting in crustaceans. In this study, changes in protein abundance in the YO were characterized over the course of a molt cycle (intermolt, early premolt, mid premolt, and late premolt) induced by multiple leg autotomy (MLA) in the blackback land crab, Gecarcinus lateralis. In all, 457 distinct protein spots were detected in the molting gland using two-dimensional gel electrophoresis, of which $230(50 \%)$ changed significantly in abundance over the course of the molt cycle (one-way permutation ANOVA, $p \leq 0.05$ ). Changes in protein abundance were most notable between the intermolt and the three premolt stages, indicative of a biological 'on-off' switch in the Y-organ. Several hemolymph species proteins, including hemocyanin, cryptocyanin, and transglutaminase, were identified which characterized physiological changes associated with molting beyond the Y-organ. An abundance of cytoskeletal proteins were identified which correspond with glandular hypertrophy and are indicative of vesicular-mediated exocytosis, possibly of ecdysteroids. Further, several proteins involved in the immune, proteostasis, and oxidative stress response are characteristic of supporting the dynamic and demanding cellular changes associated with ecdysteroidogenesis and the transition of the Y-organ from the basal to the highly active state. Many proteins involved in energetic pathways including glycolysis, the citric acid cycle, amino acid metabolism, and one-carbon metabolism changed in abundance in response to both the higher energy demands and the requirement for precursors of macromolecular synthesis of the YO over the molt cycle. Taken together, these changes in diverse physiological pathways represent the complexity involved with regulation of the Y-organ, even with just the single proposed physiological purpose of ecdysteroidogenesis.

Key Words: Gecarcinus lateralis, molting, Y-organ, energy metabolism, ecdysteroids 


\section{ACKNOWLEDGMENTS}

Financial support for this project was provided by NSF-IOS 1257732 awarded to Dr. Donald Mykles and Co-PIs Dr. Lars Tomanek, Dr. David Durica and Dr. Ernest Chang. Further financial support was provided through Cal Poly College Based Fees for supplies and travel to conferences to present this research. This project would not have been possible without these funding sources.

I would like to express my deepest gratitude Dr. Donald Mykles for his mentorship and guidance over the past seven years. Dr. Mykles provided me with the opportunity to explore molecular research when I was a freshman at Colorado State University, and has continued to do so even long after my graduation. His unceasing support and confidence in my skills have helped me to grow into the scientist I am today. Thank you to my advisor

Dr. Lars Tomanek for his support, encouragement, and understanding of my "fiercely independent" spirit. Thank you to my committee member, Dr. Kristin Hardy, for her support and patience during this process.

Fellow members of the Environmental Proteomics Lab were greatly important to my success; a huge thanks to Josh Mier, Corey Elowe, Christina Vasquez, Jackie Campbell, Mark Hamer, and Daniela Martinez for their camaraderie. I would also like to thank STAR fellow Andrea Reider and Bridges to Baccalaureate scholar Jorge Del Pozo for their help with laboratory processing. Thank you to Sunetra Das and Lindsay Martin of Colorado State University for providing the Gecarcinus lateralis transcriptomic database and guiding me through its ins-and-outs. Additionally, various support staff in the Cal Poly Biology Department were crucial to my success in too many ways to count; thank you to 
Alice Hamrick, Dave Clendenen, Tony Frazier, Kathy Simon, Ellen Calcagno, Melanie Gutierrez, and Kristen Reeves.

Finally, I would like to thank my emotional-support staff, my friends and family. Thank you to my parents, Gil and Nina Head, for their support in my research even when they don't always understand what it is I do or why I do it. Thank you to my dear friend Jessica Adinolfi for quickly taking me under her wing from day one of the Masters' program and for introducing me to nearly everyone I have ever met in SLO. And of course, finishing this work would not have been possible without being able to end each day in a home filled with love, thank you to Maggie Jenkins, Michelle Cooke, and of course Coral Cooke for their love and support. 
LIST OF TABLES.

CHAPTER

I. INTRODUCTION

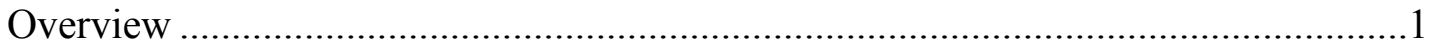

Gecarcinus lateralis Ecology and Physiology ........................................................2

Ecdysis in Crustaceans: Physiological Changes Over the Molt Cycle .........................9

Ecdysis in Crustaceans: Molecular Control and Regulation .......................................20

Socioeconomic Impacts and Relevance of Molting in Crustaceans............................29

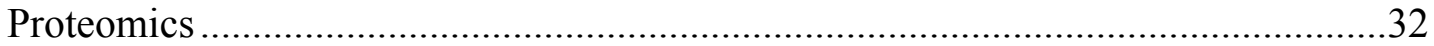

Proteomics as a Hypothesis-Generating Tool .....................................................32

Proteomic Analysis: Protein Isolation and Quantification ..................................34

Proteomic Analysis: Protein Separation and Analysis .........................................37

Proteomic Analysis: Protein Identification .........................................................40

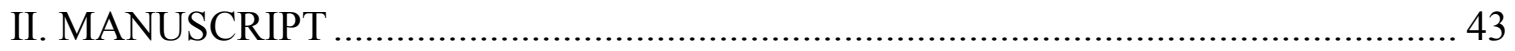

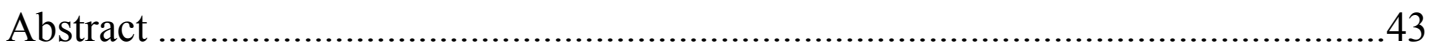

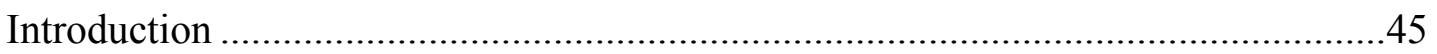

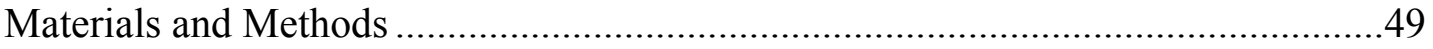

Animal Collection and Husbandry..............................................................49

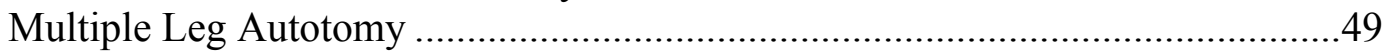

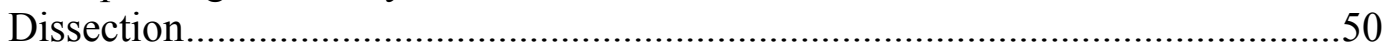

Homogenization and Protein Isolation.................................................................51

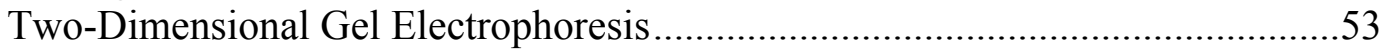

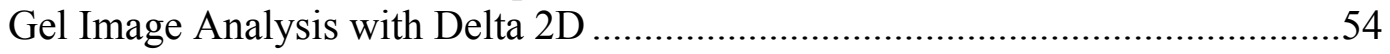

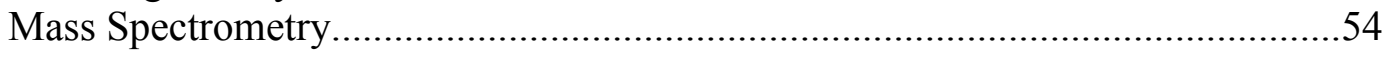

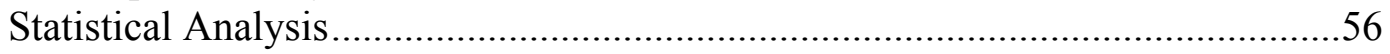

Results and Discussion .........................................................................................5

Protein Detection, Identification, and Abundance Changes ..................................57

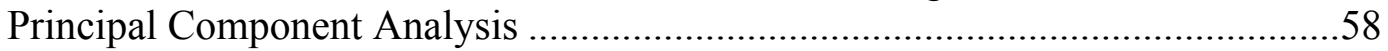

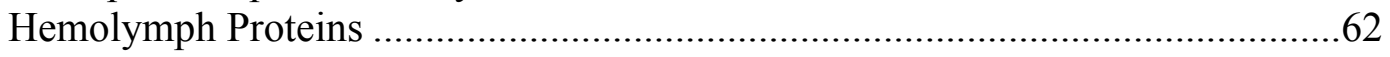

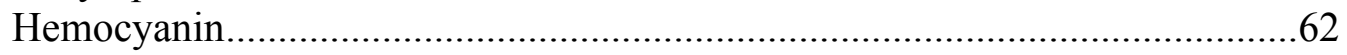

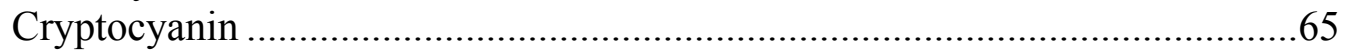

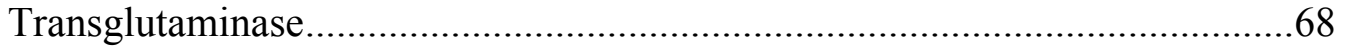

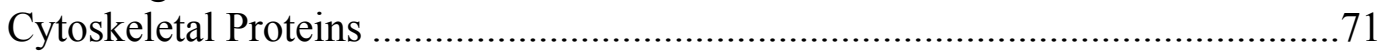

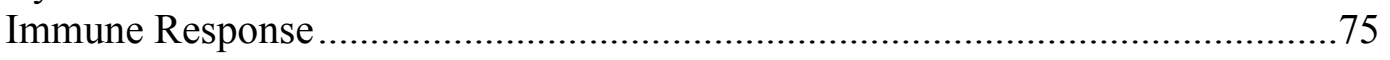

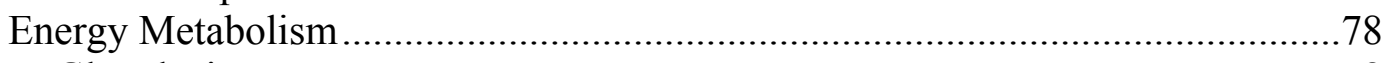

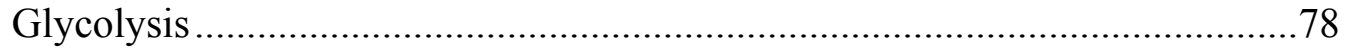

The Citric Acid Cycle ................................................................................. 80

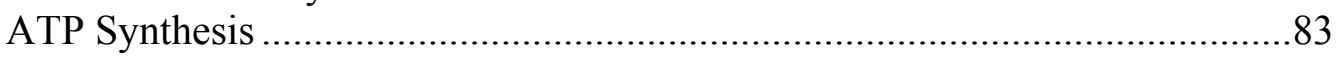




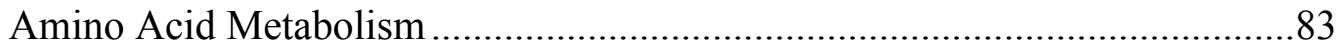

One Carbon Metabolism ................................................................................ 85

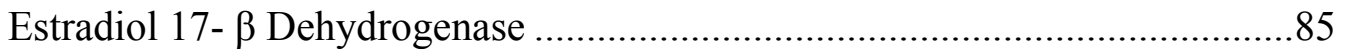

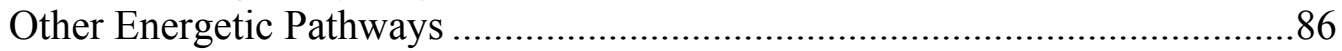

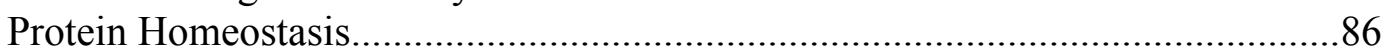

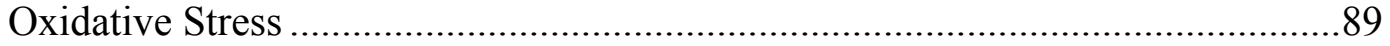

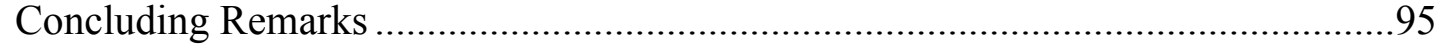

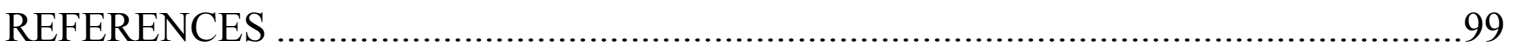

\section{APPENDIX}

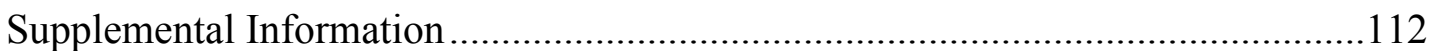




\section{LIST OF TABLES}

Table 1. Summary of molt stage names and abbreviations, and their associated indicators

Table 2. Organization of YO samples into biological replicates of groups of three YO-pairs. Samples were pooled according to molt stage first according to R-index, followed by similar hemolymph ecdysteroid values

Table 3. Positive and negative component loading values for all proteins contributing

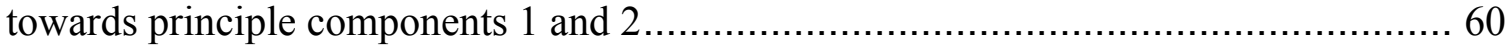

Table 4. Positive and negative component loading values for all significant proteins

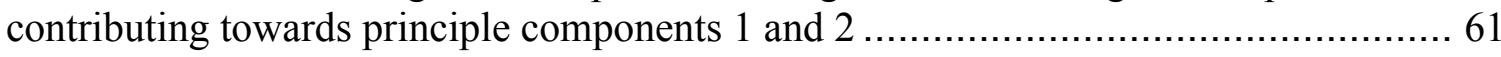

Table 5. Identified proteins within the $\mathrm{Y}$-organ of Gecarcinus lateralis in response to a molt cycle induced by multiple leg autotomy 


\section{LIST OF FIGURES}

Figure 1. Distribution map of the blackback land crab, Gecarcinus lateralis. The crab inhabits coastal regions from the tip of Florida through the Caribbean islands, from South Padre Island, TX, USA through Central America and on to French Guiana, and along the Pacific Ocean from Mexico to Colombia.

Figure 2. The blackback land crab, Gecarcinus lateralis. A. Dorsal view, showing The carapace which covers much of the organism's body cavity B. Anterior view, Showing eyestalks, chelae and mandibles $\mathrm{C}$. Chelae displaying characteristic brown-tip of dactyl segments. Artistic rendering by the author

Figure 3. Cytology of the integumentary tissue of Gecarcinus lateralis over the Course of the molt cycle. Artist rendition by the Talia Head, adapted from

Skinner, 1962

Figure 4. Cross section of a crustacean eyestalk, highlighting the location of the neurosecretory X-organ (XO) and sinus gland ( $\mathrm{SG}$ ). Other abbreviations: RN, retina; LG, lamina ganglionaris; ME, medulla externa; MI, medulla interna; MT, medulla terminalis; $S G T$, sinus gland tract. Artistic rendering by the author

Figure 5. Dorsal view of the internal anatomy of Gecarcinus lateralis, with inset (dashed lines) highlighting the location of the Y-organ (YO) on the cephalothorax. The digestive gland (hepatopancreas) is not shown to allow for visualization of other aspects of the internal anatomy. Abbreviations: ES, eyestalk; B, epibranchial areas; E, branchial epithelium; AP, anterior proventriculus; G, gills; H, heart; P, pericardial sacs; S, external setae. Artistic rendering by the author, based on Bliss, 1968 and photographic images provided by Nada Rifai (Colorado State University) ......14

Figure 6. Molt cycle related changes in YO state, ecdysteroidogenic capacity, and sensitivity to MIH over the molt cycle. Adapted from Das and Mykles, 2016.

Figure 7. MIH binds to specific transmembrane receptors on cells of the YO resulting in a signaling cascade beginning with the conversion of ATP to cAMP by adenylyl cyclase. Increasing intracellular cAMP concentrations mediate protein kinase A (PKA) activity resulting in the opening of $\mathrm{Ca} 2+$ channels on the cell surface. This leads to the activation of nitric oxide synthase (NOS) through calmodulin (CaM) and calcineurin $(\mathrm{CaN})$. Guanylyl cyclase-I (GC-1), which converts GTP to cGMP, is dependent on NO. As a result, protein kinase $\mathrm{G}$ activity increased and inhibits ecdysteroidogenesis at the transcriptional and translational level. The major downstream transcriptional and translational regulator being inhibited is hypothesized to be the mTOR complex. (Adapted from Chang and Mykles, 2011) 
Figure 8. Proposed signaling mechanisms affecting the activation state of mTOR in the crustacean YO (Adapted from Abuhagr et al., 2016)

Figure 9. The first stage of ecdysteroid synthesis in the crustacean YO begins with the conversion of cholesterol to 7-Dehydrocholesterol by a 7,8-Dehydrogenase encoded by the Halloween gene, Neverland $(N v d)$. The following reactions are termed the owing reactions are o 7-Dehydrocholesterol non-molting glossy/shroud, Spook/Spookier, and Spookiest. (Adapted from Mykles, 2011) .26

Figure 10. The second stage of ecdysteroid synthesis occurs in the Y-organ and the peripheral tissues and involves enzymes encoded by the Halloween genes, Disembodied, Phantom, Shadow, and Shade. (Adapted from Mykles, 2011).......28

Figure 11. Composition of biological replicates used for analysis of each molting stage. YO pairs from each animal were pooled with animals of similar hemolymph ecdysteroid titers for early, mid, and late premolt stages to produce 4 biological replicates for each molt stage.

Figure 12. Two-dimensional gel proteome map composed of gels from all molt stages. Spot numbers represent all 457 protein spots detected from the G. lateralis $\mathrm{YO}$ in intermolt, early, mid, and late premolt stages. The average pixel density from this fused image was normalized to determine changes in pixel density (protein abundance) among each gel image in each molt stage. All protein spots were manually excised from gels for identification using MALDI-ToF/Tof mass spectrometry, of which 191 were positively identified.

Figure 13A. Principal component analysis displaying all proteins within the Y-organ of Gecarcinus lateralis over the course of the molt cycle induced by MLA. Individual points represent a single composite gel image composed of one biological replicate (3 pairs of Y-organs from animals in the same molting stage). Points are color coordinated according to stage within the molt cycle, and clustered based on similarities in protein abundance among each gel image. Together, PC1 and PC2 explain $40.653 \%$ of the variation in protein abundance observed among molt stages. Individually, PC1 separates the intermolt stage from the premolt stages and explains $29.996 \%$ of the variation in protein abundance observed. PC2, which separates the early, mid, and late premolt stages, explains $10.657 \%$ of the variation in protein abundance seen in the $\mathrm{Y}$-organ

Figure 13B. Principal component analysis displaying all significant proteins within the Y-organ of Gecarcinus lateralis over the course of the molt cycle induced by MLA (One-way permutation ANOVA; $p<0.05$ ). This PCA represents 57.852\% of the variation in protein abundance observed among the intermolt, early, mid, and late premolt stages. Individual proteins which contribute to the positive and negative separation among both component 1 and component 2 are indicated in Table 4 
Figure 14. Hierarchal clustering using Pearson's correlation depicting relative protein abundance (normalized spot volume) for all detected hemocyanin proteins within the Y-organ of Gecarcinus lateralis. Check marks associated with individual protein spots indicate proteins which changed significantly in abundance across the molt stages and exhibit a strong contribution towards the separation among molt stages displayed along the respective component axis (see Fig. 13B and Table 4)

Figure 15. Hierarchal clustering using Pearson's correlation depicting relative protein abundance (normalized spot volume) for all detected cryptocyanin proteins within the Y-organ of Gecarcinus lateralis. Check marks associated with individual protein spots indicate proteins which changed significantly in abundance across the molt stages and exhibit a strong contribution towards the separation among molt stages displayed along the respective component axis (see Fig. 13B and Table 4)

Figure 16. Hierarchal clustering using Pearson's correlation depicting relative protein abundance (normalized spot volume) for all detected transglutaminase within the Y-organ of Gecarcinus lateralis. Check marks associated with individual protein spots indicate proteins which changed significantly in abundance across the molt stages and exhibit a strong contribution towards the separation among molt stages displayed along the respective component axis (see Fig. 13B and Table 4)

Figure 17. Levels of transglutaminase proteins in the YO of $G$. lateralis over the course of the molt cycle. Spot volumes were obtained by normalizing against the volume of all proteins and show means \pm s.e.m. $(\mathrm{N}=4)$. Molt stages that do not share a letter within individual graphs are significantly different (Tukey’s HSD). Spot numbers are shown in parentheses.

Figure 18. Hierarchal clustering using Pearson's correlation depicting relative protein abundance (normalized spot volume) for all detected cytoskeletal proteins within the Y-organ of Gecarcinus lateralis. Check marks associated with individual protein spots indicate proteins which changed significantly in abundance across the molt stages and exhibit a strong contribution towards the separation among molt stages displayed along the respective component axis (see Fig. 13B and Table 4)

Figure 19. Hierarchal clustering using Pearson's correlation depicting relative protein abundance (normalized spot volume) for all detected immune proteins within the Y-organ of Gecarcinus lateralis. Check marks associated with individual protein spots indicate proteins which changed significantly in abundance across the molt stages and exhibit a strong contribution towards the separation among molt stages displayed along the respective component axis (see Fig. 13B and Table 4) 
Figure 20. Hierarchal clustering using Pearson's correlation depicting relative protein abundance (normalized spot volume) for all detected energy metabolism proteins within the $\mathrm{Y}$-organ of Gecarcinus lateralis. Check marks associated with individual protein spots indicate proteins which changed significantly in abundance across the molt stages and exhibit a strong contribution towards the separation among molt stages displayed along the respective component axis (see

Fig. 13B and Table 4)

Figure 21. The malate-aspartate shuttle of the mitochondria. Malate is shuttled into the matrix of the mitochondria to regenerate reducing equivalents which supply the electron transport chain. Malate is subsequently converted to aspartate, and shuttled back into the cytosol. The conversion of aspartate to malate within the cytosol regenerates $\mathrm{NAD}^{+}$which serve to maintain glycolysis

Figure 22. Schematic representation of the major energetic enzymes identified within the YO of $G$. lateralis. Identified enzymes are indicated in blue, points of amino acid synthesis are indicated in red, and mTOR activation is indicated in green. Abbreviations are as follows: $\mathrm{ADH}$, aspartate dehydrogenase; $\mathrm{AK}$, arginine kinase, ASS; argininosuccinate synthetase; EN, enolase; GAPDH, glutamate dehydrogenase; IDH, isocitrate dehydrogenase; $\mathrm{MDH}$, malate dehydrogenase; PGK, phosphoglycerate kinase; TPI, triosephosphate isomerase

Figure 23. Hierarchal clustering using Pearsone kinase; TPI, triosephosphate iso protein abundance (normalized spot volume) for all detected proteins involved in protein homeostasis within the Y-organ of Gecarcinus lateralis. Check marks a ssociated with individual protein spots indicate proteins which changed significantly in abundance across the molt stages and exhibit a strong contribution towards the separation among molt stages displayed along the respective component axis (see Fig. 13B and Table 4)

Figure 24. Levels of manganese superoxide dismutase proteins in the YO of $G$. lateralis over the course of the molt cycle. Spot volumes were obtained by normalizing against the volume of all proteins and show means \pm s.e.m. $(\mathrm{N}=4)$. Molt stages that do not share a letter within individual graphs are significantly different (Tukey's HSD). Spot numbers are shown in parentheses.

Figure 25. Hierarchal clustering using Pearsonarenthesestion depicting relative protein abundance (normalized spot volume) for all detected oxidative stress proteins within the Y-organ of Gecarcinus lateralis. Check marks associated with individual protein spots indicate proteins which changed significantly in abundance across the molt stages and exhibit a strong contribution towards the separation among molt stages displayed along the respective component axis (see Fig. 13B and Table 4). 
Figure 26. Classification of major functional categories contributing to each molt stage in the Y-organ of Gecarcinus lateralis. Inner circles represent the molt stage abbreviation and activation state of the Y-organ. Proteomic analysis of ecdysal and postmolt crabs have yet to be conducted.

Figure 27A. Hierarchal clustering using Pearson's correlation depicting relative protein abundance (normalized spot volume) for Cluster I of all identified proteins observed within the Y-organ of Gecarcinus lateralis. Yellow boxes indicate a relative increase in protein abundance from the average, while blue boxes represent a lower than average protein abundance. Individual columns represent a single gel image composed of one biological replicate (3 pairs of Y-organs from animals in the same molting stage) for intermolt, early, mid, and late premolt stages as indicated above. Individual rows represent the standardized pattern of abundance for each identified protein. Five clusters are represented by separation between rows with a dashed line and represent groups of proteins which express similar changes in protein abundance among molt stages. Molecular weight $(\mathrm{Da})$ and isoelectric point $(\mathrm{pI})$ for each protein were determined based on the location of each protein spot within the composite gel image using Decodon Delta 2D.

Figure 27B. Hierarchal clustering using Pearsonn spot within the composite gel im protein abundance (normalized spot volume) for Cluster II of all identified proteins observed within the Y-organ of Gecarcinus lateralis.

Figure 27C. Hierarchal clustering using Pearson's correlation depicting relative protein abundance (normalized spot volume) for Cluster III of all identified proteins observed within the Y-organ of Gecarcinus lateralis

Figure 27D. Hierarchal clustering using Pearson of e) for Cluster III ofrelative protein abundance (normalized spot volume) for Cluster IV of all identified proteins observed within the Y-organ of Gecarcinus lateralis.

Figure 27E. Hierarchal clustering using Pearson of e) for Cluster IV of all ident protein abundance (normalized spot volume) for Cluster $\mathrm{V}$ of all identified proteins observed within the Y-organ of Gecarcinus lateralis.....

Figure 28A. Hierarchal clustering using Pearson of e) for Cluster V of all identi protein abundance (normalized spot volume) for Cluster I of proteins spots within the Y-organ of Gecarcinus lateralis which changed significantly in abundance with response to molt stage (One-way permutation ANOVA; $p<0.05$ ) 
Figure 28B. Hierarchal clustering using Pearson's correlation depicting relative protein abundance (normalized spot volume) for Cluster II of proteins spots within the Y-organ of Gecarcinus lateralis which changed significantly in abundance with response to molt stage (One-way permutation ANOVA; $p<0.05$ )

Figure 28C. Hierarchal clustering using Pearson's correlation depicting relative protein abundance (normalized spot volume) for Cluster III of proteins spots within the Y-organ of Gecarcinus lateralis which changed significantly in abundance with response to molt stage (One-way permutation ANOVA; $p<0.05$ ) 


\section{Overview}

\section{INTRODUCTION}

This study focuses on the physiological mechanisms controlling molting in the decapod crustacean, Gecarcinus lateralis. The blackback land crab, G. lateralis, has been prominently used in studies regarding molting since the 1950's when Dorothy Bliss first began studying the crab at the Woods Hole Marine Biological Laboratory. The large size of G. lateralis alongside the relative ease of husbandry involved with laboratory studies has made this an ideal organism for the study of physiological processes associated with molting in crustaceans for more than 60 years.

Growth in arthropods is a complex process as the animal is confined by a hardened exoskeleton. Thus, shedding of the exoskeleton is necessary for subsequent growth, a process termed ecdysis or molting. Ecdysis is under regulatory control by two endocrine glands (i) the Xorgan/sinus gland $(\mathrm{XO} / \mathrm{SG})$ complex which negatively regulates synthesis of pro-ecdysial hormones by (ii) the Y-organ (YO). When active, the YO synthesizes ecdysteroids which migrate to peripheral tissues and initiate a physiological cascade that culminates in ecdysis. Previous proteomic analysis of the YO of eyestalk ablated (ESA) G. lateralis has led to initial identification of the importance of nitric oxide synthase in suppression of the YO by the XO/SG (Lee and Mykles, 2006). Further studies of the proteomic changes involved with ecdysteroidogenesis in the YO are necessary, particularly to determine physiological changes that occur within the YO over the course of a natural molt cycle.

Advances in technology alongside the expansion of transcriptomic databases have aided in making proteomics an important exploratory tool. Proteomic analysis is particularly useful in studies assessing time courses, such as the progression through the molt cycle, and provides novel information from which further hypotheses may be generated. The goal of this study is to analyze the proteome of the $G$. lateralis YO over the course of a molt cycle induced by multiple leg 
autotomy (MLA). Interpretation of changes and patterns of expression in functional groups in relation to the molt stage and ecdysteroidogenesis are provided to assess physiological and molecular changes in the YO over the molt cycle.

This introductory chapter serves to provide the reader with ecological, physiological, and socioeconomic background in relation to molting in crustaceans, particularly focusing on $G$. lateralis. Also described are the methods and tools employed in proteomic studies, with special attention paid to those used in this study. Because the YO is the source of ecdysteroids, and thus responsible for initiating molting, the results of this study contribute to a better understanding of the physiological mechanisms that drive crustacean development. In particular, contributions of 191 individual proteins to six major functional groups of proteins and their relation to molt stage within the YO will be discussed in detail in Chapter II.

\section{Gecarcinus lateralis Ecology and Physiology}

The blackback land crab, Gecarcinus lateralis, is a brachyuran crustacean belonging to a family of terrestrial crabs, Gecarcinidae. G. lateralis inhabits Florida, the Caribbean islands, and the adjacent mainland coasts from South Padre, Texas through Central and South America to French Guiana; and along the Pacific coast from Mexico to Peru [Fig. 1 (Bliss et al., 1978; Hartnoll, 1988)]. They are typically found in sandy scrublands and vegetative coastal hammocks between 3 to 10 meters above the high tide line (Bliss et al., 1978; Bright and Hogue, 1972). This region provides a substrate that is rich in calcium deposits and maintains significant interstitial moisture accrued from daily dew, which aids in osmoregulation as well as stabilizes the structure of burrows (Bliss et al., 1978; Wolcott and Wolcott, 1988). Burrows for G. lateralis typically descend at about $40^{\circ}$ angles from the surface for a linear distance of about $65 \mathrm{~cm}$ (Bliss, 1979). 


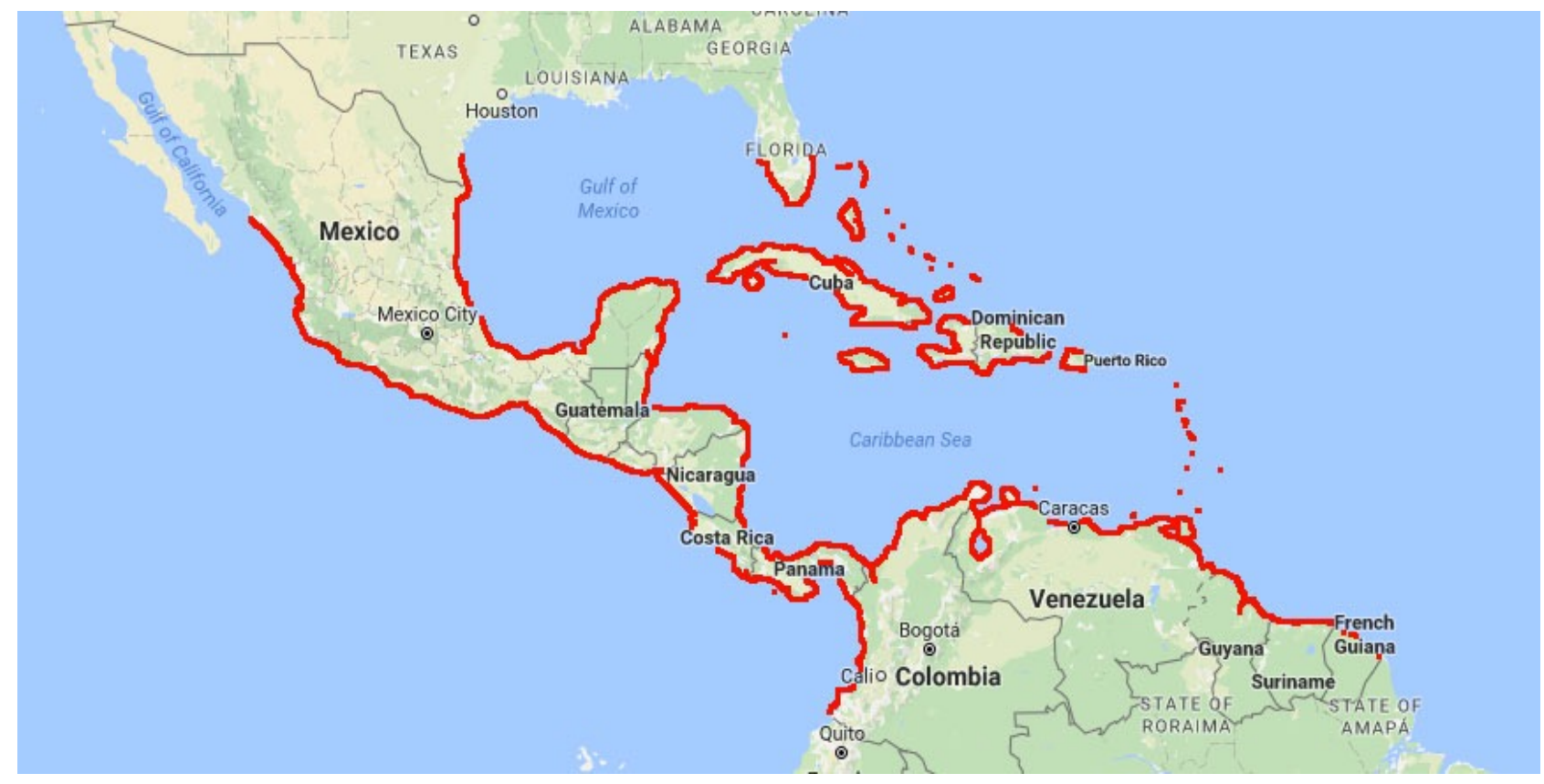

Figure 1. Distribution map of the blackback land crab, Gecarcinus lateralis. The crab inhabits coastal regions from the tip of Florida through the Caribbean islands, from South Padre Island, TX, USA through Central America and on to French Guiana, and along the Pacific Ocean from Mexico to Colombia.

Crabs are found individually in their burrows, thus burrow density can also be used as a proxy for crab density (Capistrán-Barradas, et al., 2003). Crab density varies by population as well as by topographical location. Local distribution of crabs is affected by several biotic and abiotic variables including age of vegetative growth, relative humidity, shade, and size or age class of the crabs. Increased densities of burrows tend to be observed in areas with high amounts of leaf litter, such as is typically found in old growth vegetation. Additionally, ability to retain humidity and encounter milder temperatures is typically found in older growth vegetation where shade is provided by the mature, leafy plants. Larger crabs tend to be found in greater abundance in young growth vegetation while younger, smaller crabs are more densely distributed in the old growth regions. This may reasonably be due to differences in overall metabolism of the crustaceans, with 
higher metabolic rates associated with smaller carapace size, and thus a higher energetic demand that can easily be filled by the increased leaf litter found in old growth vegetation (Herreid and Full, 1988; Capistrán-Barradas, et al., 2003).

Activity of G. lateralis is highly dependent upon two environmental variables: temperature and moisture. Although slight variations may occur among populations, the crabs are typically most active during mild temperatures ranging from about 18.3 to $22.7^{\circ} \mathrm{C}$, and activity outside of the burrow ceases when temperatures drop below about $18{ }^{\circ} \mathrm{C}$ or are in excess of $27{ }^{\circ} \mathrm{C}$. During hot summer months, this means that peak activity of the crabs typically occurs around dawn and dusk. Depending upon the specific inshore location of burrows, crabs may become active during differing times of day based on shade provided by surrounding vegetation and density and activity of predators, but in general crabs are active around dawn and dusk, and remain in their burrows during the day (Bliss et al., 1978; Bliss 1979). On the other hand, the dry and cooler winter months result in a cessation of above-ground activity until temperatures return to favorable conditions and moisture, beginning the form of a large rainfall, is abundant (Thomas, 1974).

G. lateralis are omnivorous crabs; they are typically found feeding on vegetative matter, although they have been observed to consume animal matter when it is accessible. During the summer months, the blackback land crab can be found feeding on leafy material and local fruits during foraging excursions outside of the burrow. Further, individuals have been observed feeding on freshly hatched sea turtles, young or injured birds, lizards, as well as others of their own species (Skinner et al., 1978; Erhardt and Niaussat, 1970). In preparation for the winter months, crabs can be seen carrying leafy material back into their burrows which they use to create a plug just near the end of the burrow. This plug may serve as both a food source and aid in temperature and moisture regulation during the cooler, dry winter months (Skinner, et al., 1978). 
Mating in G. lateralis typically occurs during the late summer/early fall months of August and September, although the reproductive season may extend from March to November depending on environmental conditions (Bliss et al., 1978; Klaassen, 1975). Copulation must take place within 6 hours to 9 days immediately prior to ovulation for successful fertilization and development of eggs (Klaassen, 1975). Ovary maturation and thus subsequent reproductive behaviors may be loosely correlated to lunar cycles (Klaassen, 1975). Fertilization occurs in hardshell, intermolt females, in contrast to the soft-shell postmolt fertilization that must take place in their marine counterparts (Bliss et al., 1978). Each brood pouch contains between 20,000 to 100,000 fertilized eggs, which are attached to ovigerous hairs on the females' pleopods, and held along the abdomen. Eggs develop in these brood pouches for 15-16 days after fertilization, during which time the female remains in her burrow. When larvae are ready to emerge from their egg cases, the female makes the relatively short trek from her burrow to the beach, guided by light and vibrations that originate from the surf. The females then vigorously shake to release their eggs as the shallow water of breaking waves covers them, and the larvae subsequently emerge from the egg cases on their own (Bliss et al., 1978; Klaassen, 1975). Few studies have been conducted regarding the development of the larval phases of G. lateralis. Over the course of 17-20 days, larvae metamorphose through six zoeal stages which are characterized by development of maxillae, mandibles, and pleopods (Cuesta et al., 2007; Willems, 1982). Presumably, the crabs reemerge onto the land sometime around the megalopae or first juvenile instar based on observation of individuals discovered by Dorothy Bliss, and the development of a similar gecarcinid, Cardisoma guanhumi (Bliss, 1979).

Laboratory studies have indicated that environmental regulation of molting occurs through temperature, water availability, presence of other crabs, and light cues (Bliss and Boyer, 1964). 
Favorable temperatures for molting occur between $22-28^{\circ} \mathrm{C}$, with temperatures significantly above or below this threshold resulting in a delay of ecdysis. Water availability has also been shown to be vital in initiating the molting process. Presence of sand moistened with tap water, a sponge moistened with tap water, or a dish filled with tap water were all shown to provide a sufficient source of water to support ecdysis, while dry sand alone resulted in a temporary delay. In their study, Dorothy Bliss and Jane Boyer conducted a series of unique experiments to determine how the presence of multiple crabs affects the timing of molting. Indeed, they found that crabs were not only resistant to culminating the molting process in the presence of another crab, but would actually fight to the death, unless individual containers filled with moistened sand were present as faux burrows within the community cages. Light schedule is the fourth, but not least, environmental factor shown to regulate the timing of molting. In the laboratory, constant darkness rapidly induced molts, while exposure to some light or constant light drastically increased the interval between molts. Because the source of molt inhibiting hormone is found within the eyestalks, it is likely that circadian rhythms play a role in the timing of molting for crustaceans. It is no coincidence that the conditions which prompt molting in the laboratory mimic those that are found in the burrows of G. lateralis. Few early studies have investigated molting of G. lateralis in the field as both ecdysis and the early postmolt stages take place within the burrow to ensure a safe molt and subsequent hardening of the exoskeleton (Wolcott and Wolcott 1985; Green 2004).

Being terrestrial crabs, G. lateralis faces several unique complications associated with life on land. Radical adaptations in regards to daily and seasonal activity, molt-stage dependent reproduction, and habitat distribution which have been previously mentioned have all aided in the transition of crabs from sea to land, and allow them to be almost completely independent of aquatic systems. The gills in land crabs are reduced in size and are specialized for aerial respiration. An 
enlarged branchial chamber with a respiratory epithelium aids in respiration (Taylor and Davies, 1981). High oxygen tensions across respiratory surfaces alongside the high oxygen capacity of the hemolymph compensates for the reduced gill size (Taylor and Davies, 1981). One of the most obvious challenges faced by terrestrial crabs is that of water retention. Remarkably, individuals of G. lateralis can withstand up to $21 \%$ loss of total body water (Bliss, 1968). Fortunately, however, they have adapted several mechanisms to acquire and maintain moisture, even in the sometimes arid regions they inhabit. As previously mentioned, moisture heavily influences their seasonal and daily activities, specifically with daily dews playing a direct role in moisture retention in crabs. In fact, setae which extend to the ventral exterior of the crab from pericardial sacs are vital in conducting droplets of moisture from dew within the burrows into the branchial chambers and pericardial sacs via capillary action [(Fig. 5) Bliss, 1979]. This capillary conduction of water is used to keep the gills moist, where actively transported salts are reabsorbed from the urine alongside passively transported water (Bliss, 1979; Wolcott, 1991). Water collection and conduction by the pericardial sacs is also vital in the molting process. During late premolt, increased water retention causes bloating in the pericardial sacs, which aids in the separation of the old exoskeleton along the posterior of the crab. Alternating contraction of the two pericardial sacs during the actual process of ecdysis act as 'hydraulic pumps' to assist the crab in emerging from the confines of the old, hardened exoskeleton (Mason, 1970). Finally, the water collected and retained by the pericardial sacs serves to stretch the new, soft exoskeleton, ensuring growth of the animal after ecdysis, and provides structural support which maintains the soft exoskeleton in its proper shape as it becomes hardened (Bliss, 1979, Taylor and Kier, 2003) This temporary hydrostatic skeleton allows crabs to maintain mobility after ecdysis, despite the soft state of the newly exposed exoskeleton (Taylor and Kier, 2003; Taylor and Kier, 2006). 


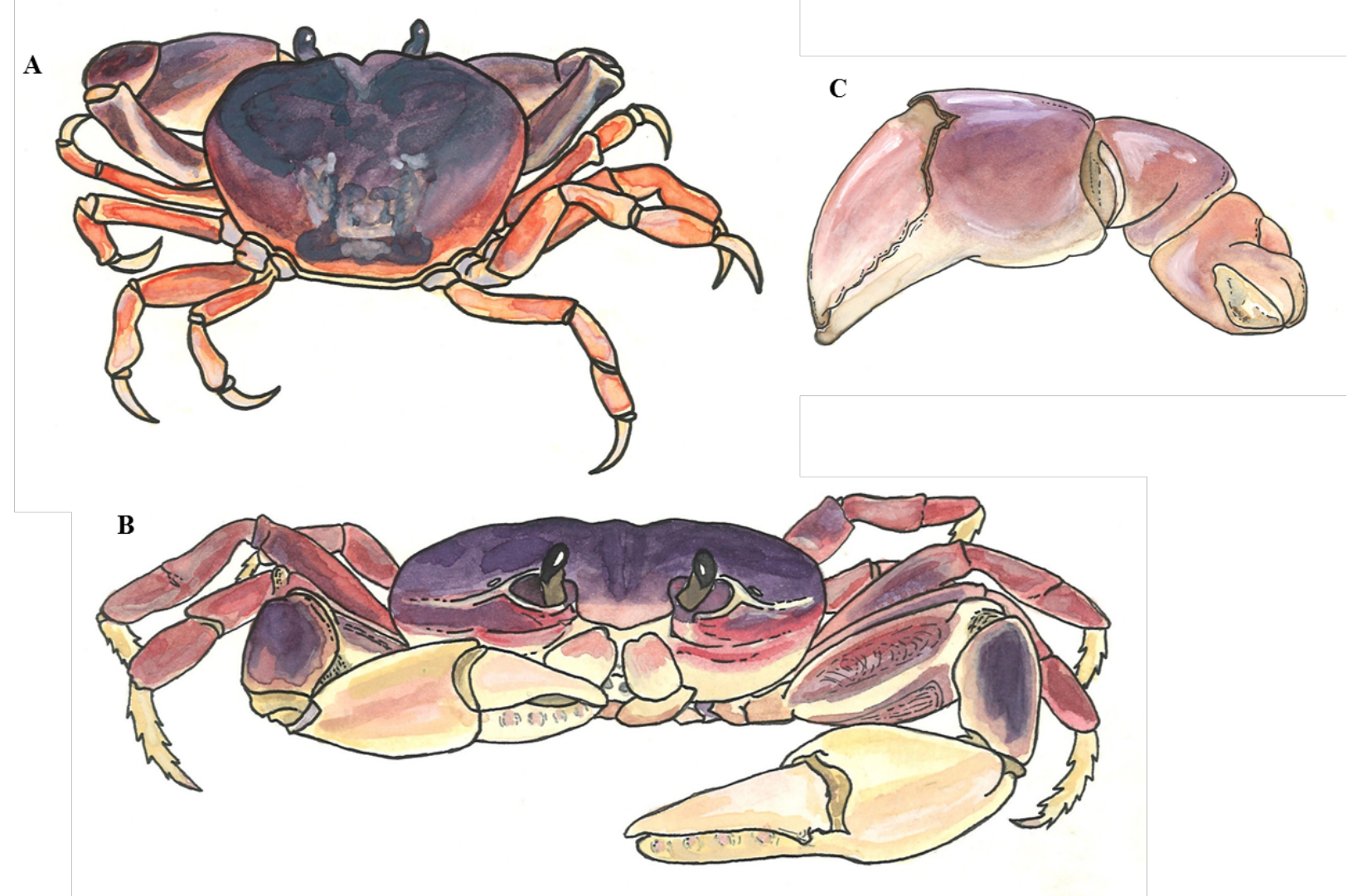

Figure 2. The blackback land crab, Gecarcinus lateralis. A. Dorsal view, showing the carapace which covers much of the organism's body cavity B. Anterior view, showing eyestalks, chelae and mandibles C. Chelae displaying characteristic brown-tip of dactyl segments. Artistic rendering by the author.

Hand-in-hand with the effects of the terrestrial environment on crustaceans, land crabs have also been shown to have major impacts on their habitats. Land crab populations can affect recruitment of some plant species through propagule predation (Capistrán-Barradas et al., 2003). Further, consumption of leaf litter aids in the recycling of nutrients stored within the near-shore terrestrial environments inhabited by these crustaceans (Green et al. 1997; Louda and Zedler, 1985). Burrow construction also aids in soil aeration and additional breakdown and recycling of nutrients within this ecosystem (Kellman and Roulet, 1990; Kellman and Delfosse, 1993; 
Robertson and Daniels, 1989). Based on the high degree of interplay between G. lateralis, and its environment, it seems obvious that studies regarding the physiological control of molting are vital in understanding the success of this organism, and thus ecosystem health for tropical coastal dunes and hammocks.

\section{Ecdysis in Crustaceans: Physiological Changes Over the Molt Cycle}

In order to understand the process of molting, we must first be oriented to certain anatomical features of $G$. lateralis. The exterior of G. lateralis is covered by a hardened exoskeleton composed of chitin and proteins which become cross-linked during the sclerotization process. The carapace is the colorful structure which covers the dorsal side of the animal, housing the main body cavity (Fig. 2). The exoskeleton is composed of two primary segments, an outermost cuticle which is superficially calcified, and a basal living epidermis layer. The non-living portion of the exoskeleton is composed of an outermost epicuticle layer, which comes in direct contact with the environment, followed by the exocuticle, a thick endocuticle, and finally a membranous layer. Just beneath the membranous layer resides the epidermis, a thin sheet of cells which synthesize and repair the cuticular layers of the exoskeleton [Fig. 3 (Skinner, 1962)]. Below the epidermis lies connective tissue containing blood (hemolymph) sinuses, tegumental glands, reserve cells, and lipoprotein cells (Skinner et al., 1992).

The integument undergoes a major reorganization during the molting process. Apolysis, or degradation of the membranous layer and separation of the epidermis from the old exoskeleton is the first step in synthesis of a new exoskeleton during early premolt (Fig. 3). The epidermal cells then enlarge and begin to secrete a new epicuticle and exocuticle, as the old endocuticle is degraded and resorbed. As the molt cycle progresses, the old exoskeleton continues to 
be degraded as the new layers are synthesized and grow in size. In G. lateralis, calcium from the old cuticle is re-absorbed and deposited in gastroliths that line the epithelium of the cardiac stomach. At ecdysis, the crab removes itself from the old exoskeleton and emerges in a newly synthesized soft exoskeleton composed of epicuticle and exocuticle. After ecdysis, the deposited calcium is then solubilized and used for hardening of the exoskeleton (Skinner et al., 1992). Endocuticle synthesis occurs from late postmolt $(B)$ through the early stages of intermolt $\left(\mathrm{C}_{1-3}\right)$. The exoskeleton remains pliable for about 4 to 8 days immediately following ecdysis (Drach,1939). Melanization and sclerotization of the cuticle harden and further add color to the carapace as a result of tanning agents such as chitin and phenoloxidases that arrive in the hemolymph (Skinner et al., 1992; Sugumaran and Barek, 2016). Each molting event results in a 1 to $7 \%$ increase in total carapace width for G. lateralis (Skinner, 1962).

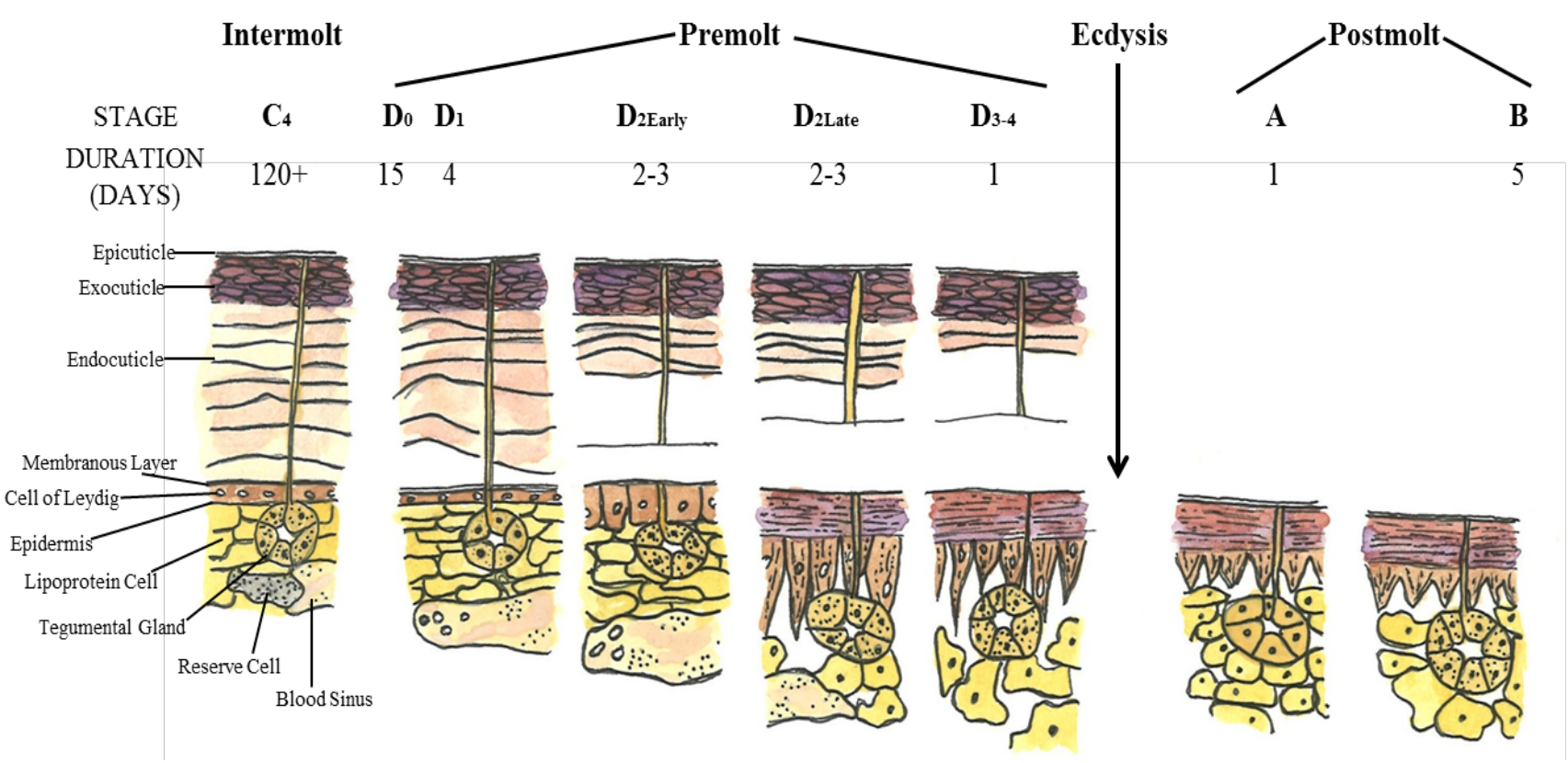

Figure 3. Cytology of the integumentary tissue of Gecarcinus lateralis over the course of the molt cycle. Artist rendition by the Talia Head, adapted from Skinner, 1962. 
Tightly coupled to synthesis of the new exoskeleton during premolt is the regeneration of lost limbs. Limb loss in decapod crustaceans readily occurs in response to injury or predation, and lost limbs are regenerated prior to the next molt (Mykles, 2001, Bliss and Boyer, 1964). Immediately following the loss of a limb, basal growth of a regenerating limb, termed a primary limb bud, occurs during the intermolt stage. This basal growth involves cell division and differentiation into basal papillae (Mykles, 2001). Once the animal enters the premolt period, rapid proecdysial growth of the primary limb bud occurs, mostly in early premolt (stage $\mathrm{D}_{0}$ ). Proecdysial limb bud growth involves protein synthesis and water uptake. Measurement of the length of a limb bud relative to the carapace width $(\mathrm{R}$ index $=$ length of limb bud $\mathrm{x}$ 100/carapace width) provides an external, non-invasive means of tracking progression through the molt cycle (Bliss and Boyer, 1964). Proecdysial limb bud growth is under stimulatory hormonal control by ecdysteroids secreted during the premolt period. Once the animal has reached ecdysis, the newly regenerated limbs unfold as the animal exits the old carapace, and continued water uptake by the limbs helps to stretch and hydrostatically support the new limb as sclerotization occurs over the next few days.

Limb loss and regeneration is useful in laboratory experiments to manipulate the timing of molting. Multiple leg autotomy (MLA) involves the loss of 5-8 walking legs and can be used to induce a precocious molt in adult land crabs, Gecarcinus lateralis. The predictable and consistent response of G. lateralis to MLA is a major reason why a large amount of research on crustacean molting has focused on the land crab over the past 60 years (Skinner and Graham, 1972). Animals molt within 6-8 weeks following MLA, during which time the lost limbs undergo basal then proecdysial growth (Mykles, 2001). MLA provides a rapid means to activate the YO into synthesizing and secreting ecdysteroids, which initiate the related premolt processes such as synthesis of a new exoskeleton, claw muscle atrophy, and limb regeneration. Essentially, MLA 
provides a means to mimic a natural molt so that studies such as the one presented in Chapter II can take place without delay. Multiple leg autotomy is in contrast to a second method of inducing molting, termed eyestalk ablation (ESA), which may not entirely represent a natural molt, and will be discussed further in the following paragraphs. Autotomy of primary limb buds can also affect the timing of molting. The loss of a primary limb bud during proecdysis, when the R index is between $7-17$, can delay ecdysis by 2 to 3 weeks so that a secondary limb may be regenerated prior to molting (Holland and Skinner, 1976). During this time, proecdysial growth of any primary limb regenerates slows or ceases until the $\mathrm{R}$ index of the secondary limb bud approximates that of the primary limb buds. Interestingly, limb bud autotomy also slows or stops other premolt processes by causing an immediate drop in hemolymph ecdysteroid titers to intermolt levels (Skinner and Graham, 1970; Yu et al., 2002). A proposed limb autotomy factor proecdysis ( $\mathrm{LAF}_{\text {pro }}$ ) has MIHlike activity and is synthesized in central neurons then transported to relevant tissues to carry out ecdysial-suspending processes (Mykles, 2001; Yu et al., 2002).

Synthesis of a new exoskeleton and the regeneration of lost limbs are all molt-cycle regulated processes. Regulation of the molt cycle is controlled by two endocrine glands. The Xorgan/sinus gland $(\mathrm{XO} / \mathrm{SG})$ is located in the eyestalks, where it can receive environmental cues associated with favorable molting conditions as described above. The XO is composed of enlarged endings of a group of neurosecretory neurons [Fig. 4 (Hanström, 1939)]. One major function of the $\mathrm{XO} / \mathrm{SG}$ involves the synthesis of molt-inhibiting hormone $(\mathrm{MIH})$, which was first described after a series of elegant experiments involving the removal of eyestalks and injection with eyestalk extracts in several crustacean species (Bruce and Chang, 1984; Chang et al., 1976; Hopkins, 1982; Keller and O'Connor, 1982). MIH is just one member of a novel neuropeptide family that has roles in regulating molting, reproduction, and metabolism (Chang and Mykles, 2011). MIH acts by 
binding to its receptor on the surface of the $\mathrm{YO}$ and inhibiting transcription of ecdysteroidogenic genes (Nakatsuji et al., 2009). Ultimately, the role of MIH, and thus the XO, in crustaceans is the inhibition of ecdysis. As previously mentioned, eyestalk ablation is a means by which the molt interval can be shortened in most decapod crustaceans as it involves the complete removal of the source of MIH. However, given the diverse functions of neuropeptides secreted from the XO, eyestalk ablation may interfere with or alter other physiological processes, causing the induced molt to not be entirely representative of a natural molt. Proteomic analysis of the G. lateralis YO in response to ESA was conducted in tandem with the research presented in Chapter II, and the excised protein spots await identification.

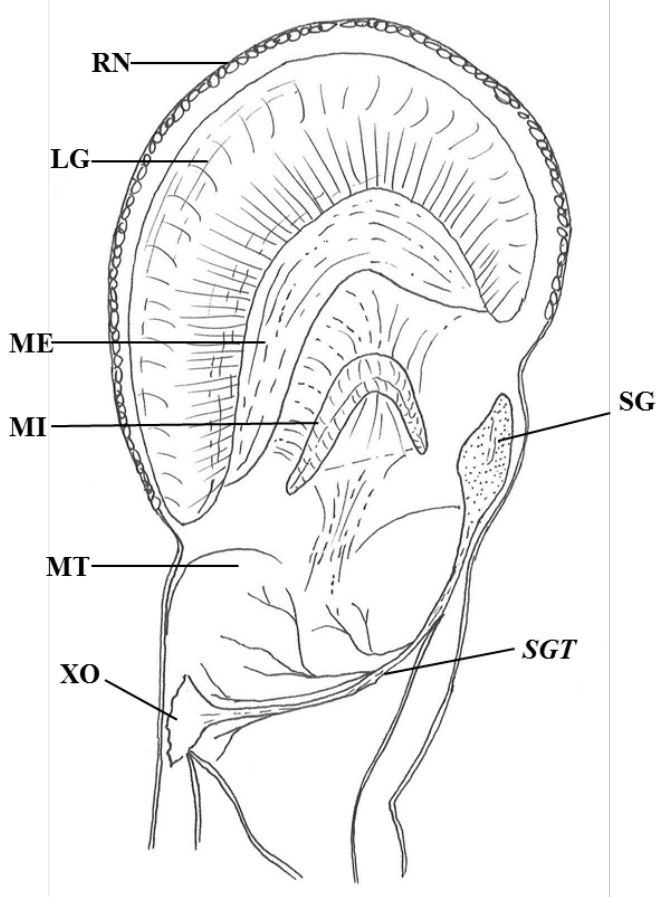

Figure 4. Cross section of a crustacean eyestalk, highlighting the location of the neurosecretory Xorgan (XO) and sinus gland (SG). Other abbreviations: RN, retina; LG, lamina ganglionaris; ME, medulla externa; MI, medulla interna; MT, medulla terminalis; $S G T$, sinus gland tract. Artistic rendering by the author. 
The molting gland, or Y-organ (YO) is the second organ involved in the regulation of molting. This paired organ is located along the branchial epithelium in the cephalothorax posterior to the mandibles on both sides (Fig. 5). Ecdysteroid synthesis and secretion is the primary function of the $\mathrm{YO}$, and activity of the $\mathrm{YO}$ is dependent upon both the presence of $\mathrm{MIH}$ and the molt stage of the animal. Ecdysteroids are a class of steroid hormones used by all arthropods to initiate processes associated with molting in peripheral tissues. Ultimately, the role of ecdysteroids, and thus the YO, in crustaceans is initiating ecdysis. Cellular and molecular description of ecdysteroid

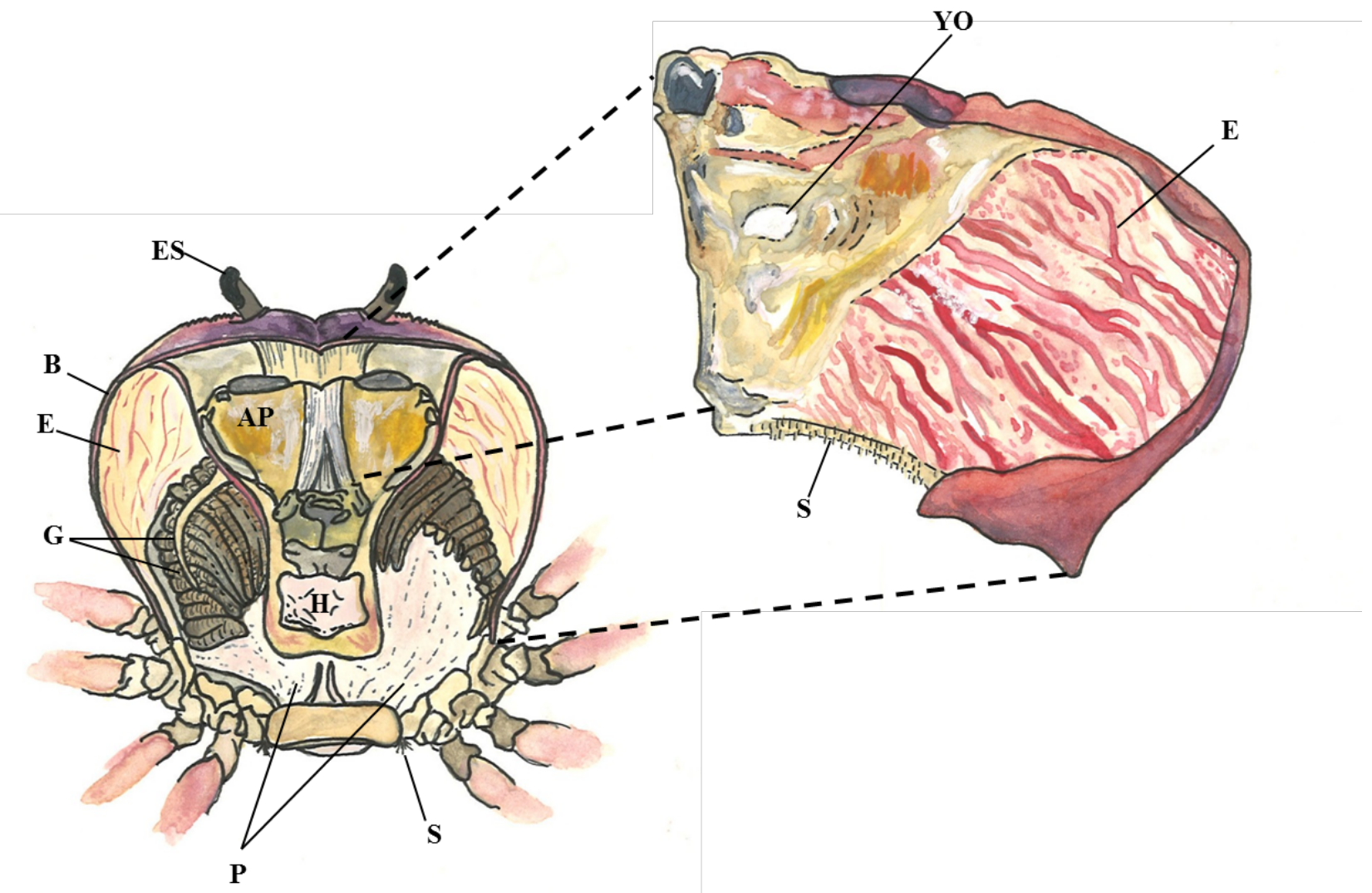

Figure 5. Dorsal view of the internal anatomy of Gecarcinus lateralis, with inset (dashed lines) highlighting the location of the Y-organ (YO) on the cephalothorax. The digestive gland (hepatopancreas) is not shown to allow for visualization of other aspects of the internal anatomy. Abbreviations: ES, eyestalk; B, epibranchial areas; E, branchial epithelium; AP, anterior proventriculus; G, gills; H, heart; P, pericardial sacs; S, external setae. Artistic rendering by the author, based on Bliss, 1968 and photographic images provided by Nada Rifai (Colorado State University). 
synthesis and mode of action will be discussed in the next section. For now, discussion of ecdysteroids and $\mathrm{YO}$ activity will focus on the changes in rates of synthesis and secretion by the YO across the molt stages.

Progression through the molt cycle involves a series of discrete steps or stages, which have been named both alphabetically (A-E), and well as descriptively (intermolt, early, mid, and late premolt, and postmolt). Passage through these molt stages can be monitored by changes in the integument and regenerating limb buds, as previously described, as well as changes in YO activity and circulating ecdysteroid titers. The following discussion will now focus on the later changes, which are ultimately responsible for the changes in the exoskeleton and limb regeneration.

The YO transitions through four physiological states- basal, activated, committed, and repressed- during the molt cycle. Due to the existence of several different names for each stage in the molt cycle, Table 1 was created to clarify and summarize the names and major aspects of each. We will begin with a brief discussion of the basal, intermolt $\left(\mathrm{C}_{4}\right)$ stage. This stage is characterized by low levels of ecdysteroid secretion from the YO, resulting in circulating hemolymph ecdysteroid titers ranging from 2-20pg/ $\mu$ l (Chang and Mykles, 2011). The presence of molt inhibiting hormone actively binds to MIH-receptors on the YO surface and suppresses high levels of ecdysteroid synthesis. The intermolt stage is characterized by little to no activity in regards to molt-related processes and physiology.

Initiation of the premolt process begins in early premolt $\left(\mathrm{D}_{0}\right)$ with a reduction in $\mathrm{MIH}$ secretion from the XO/SG [Fig. 6 (Chang and Mykles, 2011). As a result of decreased MIH, the YO begins to hypertrophy and enters an activated state which results in measurable increases YO synthesis of ecdysteroids and drastic changes in the expression of hundreds of proteins (Lee and Mykles, 2006; results of this study Chapter II). These ecdysteroids travel through the hemolymph 
to peripheral tissues where they begin to exert molt-related effects. Previously described effects of the increases in ecdysteroid titers include apolysis of the exoskeleton and proecdysial growth of limb regenerates. Further, ecdysteroids initiate formation of the previously described gastroliths which serve as calcium stores in the lining of the stomach epithelium (Ueno et al., 1992; Skinner et al., 1992).

Early premolt also denotes the beginning of somatic muscle degradation within the chelae that continues throughout the premolt stages (Skinner, 1966; Mykles 1997). Approximately 60\% of the muscle mass in the chelae is degraded prior to ecdysis in order to allow the rather large appendage to be extracted through the small joint which attaches it to the cephalothorax (Skinner, 1966). The final hallmark of the early premolt stage is that the YO remains sensitive to resupression by increased or exogenous MIH or $\mathrm{LAF}_{\text {pro. }}$.

Table 1. Summary of molt stage names and abbreviations, and their associated indicators.

\begin{tabular}{|c|c|c|c|c|c|c|c|c|}
\hline Molt Stage & $\begin{array}{c}\mathrm{C}_{4} \\
\text { Intermolt }\end{array}$ & $\begin{array}{c}\mathrm{D}_{0} \\
\text { Early Premolt }\end{array}$ & $\begin{array}{c}\quad \mathrm{D}_{1,2} \\
\text { Mid Premolt }\end{array}$ & $\begin{array}{l}\qquad \mathrm{D}_{3,4} \\
\text { Late Premolt }\end{array}$ & $\begin{array}{c}\text { E } \\
\text { Ecdysis }\end{array}$ & $\begin{array}{c}\text { A } \\
\text { Early } \\
\text { Postmolt }\end{array}$ & $\begin{array}{c}\text { B } \\
\text { Postmolt }\end{array}$ & $\begin{array}{l}\text { C } C_{1-3} \\
\text { Late Postmolt }\end{array}$ \\
\hline Description & Anecdysis & Proecdysis & Proecdysis & Proecdysis & Ecdysis & Metecdysis & Metecdysis & Metecdysis \\
\hline YO State & Basal & Activated & Committed & Repressed & - & Basal & Basal & Basal \\
\hline $\begin{array}{c}\text { Sensitivity to } \\
\text { MIH } \\
\end{array}$ & High & High & Low & Low & & High & High & High \\
\hline Exoskeleton & Full & Apolysis & \begin{tabular}{|} 
Synthesis of \\
new epicuticle \\
and exocuticle \\
Absorption of \\
old \\
endocuticle
\end{tabular} & $\begin{array}{l}\text { Synthesis of } \\
\text { new epicuticle } \\
\text { and exocuticle } \\
\text { Absorption of } \\
\text { old } \\
\text { endocuticle }\end{array}$ & Release from & $\begin{array}{c}\text { Growth and } \\
\text { expansion of } \\
\text { soft } \\
\text { exoskeleton }\end{array}$ & $\begin{array}{l}\text { Endocuticle } \\
\text { synthesis } \\
\text { Sclerotization } \\
\text { of new } \\
\text { exoskeleton }\end{array}$ & $\begin{array}{l}\text { Endocuticle } \\
\text { synthesis } \\
\text { Sclerotization } \\
\text { of new } \\
\text { exoskeleton }\end{array}$ \\
\hline $\begin{array}{c}\text { Limb } \\
\text { Regeneration }\end{array}$ & Basal Growth & $\begin{array}{l}\text { Proecdysial } \\
\text { Growth }\end{array}$ & $\begin{array}{l}\text { Proecdysial } \\
\text { Growth }\end{array}$ & $\begin{array}{l}\text { Proecdysial } \\
\text { Growth }\end{array}$ & $\begin{array}{c}\text { Release of } \\
\text { regenerated } \\
\text { limbs from old } \\
\text { exoskeleton }\end{array}$ & - & - & - \\
\hline R Index & $0-10$ & $11-15$ & $16-23$ & 23 & - & - & - & - \\
\hline
\end{tabular}


Entry into to the mid premolt stage is characterized by the transition of the Y-organ from the activated to the committed state (Fig. 6). By the end of mid premolt the Y-organ is now refractory to inhibition by exogenous sources of $\mathrm{MIH}$ or $\mathrm{LAF}_{\text {pro. This }}$ is not to say that endogenouss levels of MIH are completely absent in the mid premolt stage, but that the animal has committed to molt and will do so regardless of the loss of additional limbs, or unfavorable environmental factors. Once the animal has entered into the $\mathrm{D}_{1,2}$ stage it will typically molt within a month (Skinner, 1985a).

Both ecdysteroid synthetic capacity and hemolymph ecdysteroid titers peak in this stage [Fig. 6 (Chang and Mykles, 2011)]. This increase in circulating ecdysteroids means that they are initiating premolt responses in all peripheral tissues which possess an ecdysteroid receptor. Namely, the epithelium of the exoskeleton continues to reabsorb the old endocuticle and synthesize a new epicuticle and exocuticle. Proecdysial growth of limb buds occurs most rapidly during the early premolt stage, and growth slowly continues through mid and late premolt. Calcium absorption and deposition in the gastroliths of the stomach continues.

The last of the premolt stages, late premolt $\left(\mathrm{D}_{3,4}\right)$, is noted by a precipitous drop in circulating ecdysteroid levels [Fig. 6 (Chang and Mykles, 2011)]. This drop in hemolymph ecdysteroids may be a result of negative feedback of extremely high ecdysteroid titers on the YO (Nimitkul, 2011). Ultimately, the repressed YO is characterized by a continued insensitivity to $\mathrm{MIH}$, alongside increased excretion from the YO and decreased ecdysteroid synthesis (Chang and Mykles, 2011). The timing of the drop in circulating ecdysteroid titers appears to regulate the timing of ecdysis, with injections of exogenous ecdysteroids delaying ecdysis (Cheng and Chang, 1991). Meanwhile, the new exoskeleton continues to be secreted and atrophy of the claw muscles reaches about $40 \%$ of the intermolt muscle mass (Skinner, 1966). Limb bud R indices reach their 
maximum values in late premolt, which in G. lateralis is about 23 (Chang and Mykles, 2011). In all, the late premolt stage lasts only about a week (Skinner, 1985a).

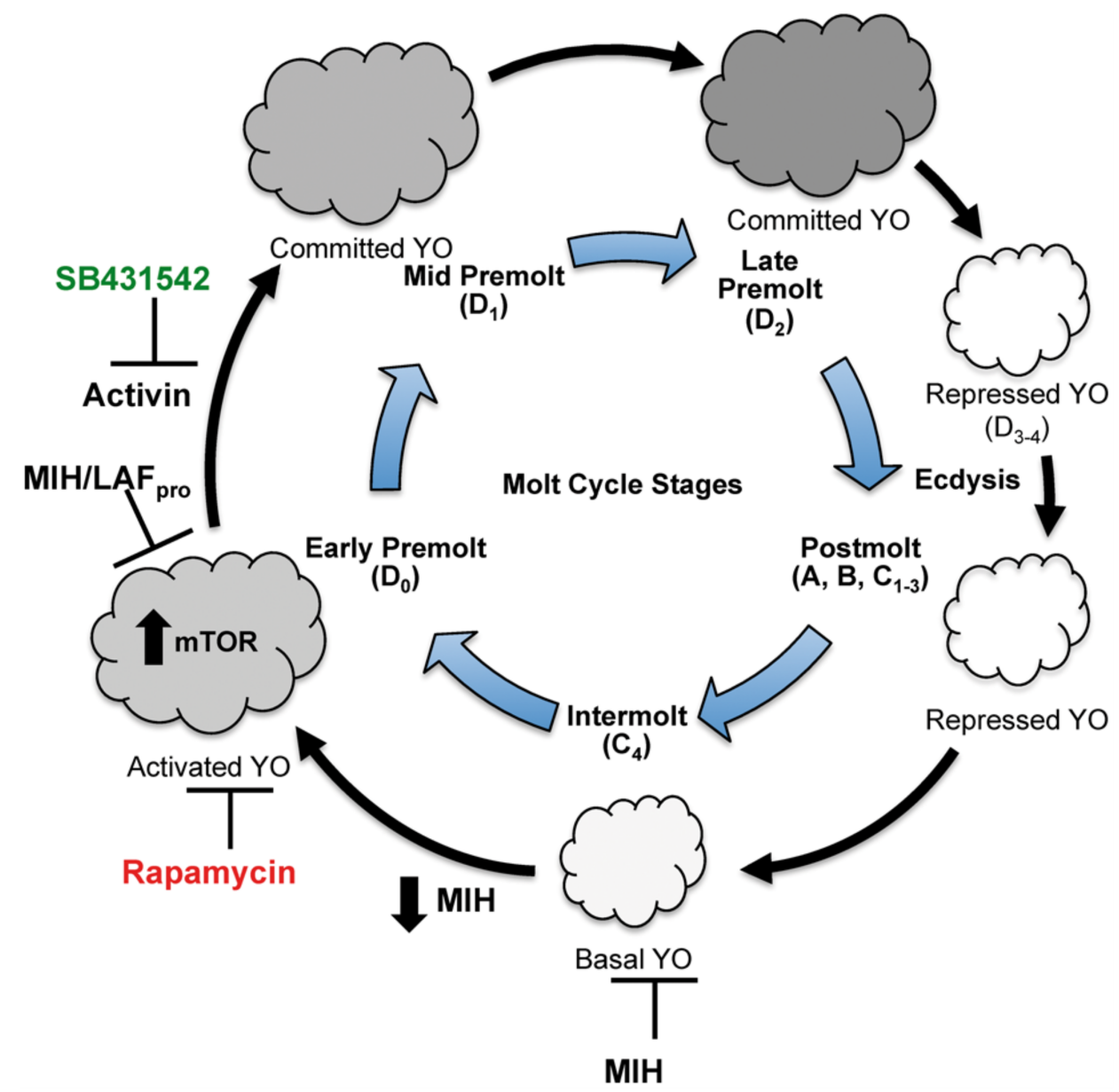

Figure 6. Molt cycle related changes in YO state, ecdysteroidogenic capacity, and sensitivity to MIH over the molt cycle. Adapted from Das and Mykles, 2016.

All of these major physiological changes and rearrangements have led up to the grand event of ecdysis, a truly fascinating phenomenon. This event can last from just a few minutes to up to several hours, depending on both the species and size of the crustacean (Bliss, 1979). Hydrostatic pressure within the cephalothorax is extremely important during and just after the molting process (Bliss, 1979; Taylor and Kier, 2003). The increased hydrostatic pressure resultant of the increase water uptake and retention during premolt is sufficient to separate the old exoskeleton at the 
epimeral suture on the caudal end of the crab (Bliss, 1979; Taylor and Kier, 2003). Through this separation or crack, the animal is able to back directly out of the old exoskeleton, first removing the body cavity and walking legs, followed by the chelae, and finally the lining of the foregut (Skinner, 1979). As previously mentioned, peristaltic contraction of the pericardial sacs (Fig. 5) facilitates the withdrawal of the animal from its old exoskeleton.

As soon as ecdysis has completed, the animal has now entered the postmolt stages. The first of the postmolt stages, A, is also called the soft-shell stage as the carapace remains pliable and is fully supported, and initially stretched, by internal hydrostatic pressure (Taylor and Kier, 2003; Skinner, 1985b). This stage typically only lasts a few hours to days as the crab remains vulnerable to predation and has limited mobility. Transition into the successive postmolt stages, B and $\mathrm{C}_{1-3}$, is characterized by continued sclerotization of the exoskeleton and secretion of the endocuticle (Bliss and Boyer, 1964). During this time, the YO atrophies and remains repressed, and sensitivity to MIH resumes (Das and Mykles, 2016). Additionally, muscle synthesis in the chelae increases and returns muscle mass to normal levels.

Succession into the intermolt, stage $\mathrm{C}_{4}$, stage marks the end of another round of growth. The YO returns to the basal state, synthesis and secretion of the exoskeleton is complete, and lost limbs have been regenerated. The crab may now resume activities such as foraging for food and searching for potential mates. This concludes our discussion on the basic physiology of crustacean molting. Although special attention was paid to G. lateralis, comparative research over the past decade has shown that this process of growth is highly conserved across crustaceans (Chang and Mykles, 2011; Chang, 1995). The focus of the next section will cover in more depth the cellular and molecular processes involved in regulation of molting within the X-organ and YO specifically. 


\section{Ecdysis in Crustaceans: Molecular Control and Regulation}

The molecular regulation of molting is tightly regulated in order to precisely control the physiological changes and restructuring that occurs as crustaceans grow, or molt. Regulation of molting processes in crustaceans are primarily controlled by ecdysteroids which are C-27 steroid hormones (Lachaise et al., 1993, Mykles, 2011). The synthesis of these ecdysteroids within the Yorgan is under direct negative regulation by the presence of molt inhibiting hormone (MIH) secreted from the X-organ/sinus gland. This section will focus on the production and cellular mode of action of molt inhibiting hormone, and the specific pathways within the YO that are associated with ecdysteroidogenesis.

Synthesis of MIH takes place in the neurosecretory neurons of the crustacean eyestalk called the $\mathrm{XO}$, while storage and release of $\mathrm{MIH}$ takes place in the neural termini located in the sinus gland [Fig. 4 (Chang, 1995)]. MIH is a neuropeptide hormone composed of approximately 74-79 amino acids (Nakatsuji et al., 2009). Contradicting evidence exists between whether changes in MIH peptide within the sinus gland occurs over the molt cycle (Nakatsuji et al., 2009). Analysis of the crayfish, Procambarus clarkii, sinus gland showed a 2-fold increase in MIH peptide within the sinus gland in early and mid premolt relative to intermolt. These results suggest a decrease in secretion of MIH from the sinus gland during these premolt stages, while synthesis remains relatively constant resulting in the observed accumulation of MIH peptide (Nakatsuji et al., 2000). However, analysis of the green shore crab, Carcinus maenas, showed no changes in MIH peptide content within the sinus gland at any given stage of the molt cycle (Chung and Webster, 2003). Hemolymph titers of MIH peptide also contrast between these two species. As such, further research on changes in synthesis and circulation of MIH on multiple species is needed to clarify this important aspect of molting regulation. 
Excretion of $\mathrm{MIH}$ from the $\mathrm{XO} / \mathrm{SG}$ occurs directly into the hemolymph where it is then transported to target organs, such as the YO (Chang, 1995). MIH is released into the hemolymph in pulses, with each pulse having a relatively short half-life of 5-10 minutes. This results in relatively low circulating hemolymph MIH titers throughout intermolt (Chung and Webster, 2005). Binding of MIH to its receptor, a putative G-protein coupled receptor, on the surface of the YO initiates a intracellular cascade of cyclic nucleotide second messengers which inhibits various levels of ecdysteroid synthesis (Chang and Mykles, 2011). MIH signaling within the cells of the YO can be divided into two phases, the $\mathrm{cAMP} / \mathrm{Ca}^{2+}$-dependent "triggering" phase, and the NO/cGMP-dependent "summation" phase [Fig. 7 (Chang and Mykles, 2011)].

The triggering phase first involves the conversion of ATP to cAMP by adenylyl cyclase (Lee et al., 2007). This transient increase in cAMP triggers an influx of calcium by means of activation of protein kinase $\mathrm{A}(\mathrm{PKA})$; calmodulin $(\mathrm{CaM})$ is subsequently activated by intracellular $\mathrm{Ca}^{2+}$. Calcineurin $(\mathrm{CaN})$ is a protein phosphatase activated by $\mathrm{Ca}^{2+} / \mathrm{CaM}$ (Kone, 2001). Such activation of $\mathrm{CaN}$ marks the switch from the "triggering" phase to the "summation" phase (Chang and Mykles, 2011). The first step of summation is the dephosphorylation, thus activation, of nitric oxide synthase (NOS). Sustained activation of NOS may also occur through direct binding of $\mathrm{Ca}^{2+} / \mathrm{CaM}$ to NOS (Covi et al., 2008; McDonald et al., 2011). Nitric oxide (NO) in turn activates a soluble guanylyl cyclase (GC-1) which synthesizes the signaling molecule, cGMP, from GTP. It is this sustained activation of GC-I which provides a mechanism for continuous suppression of the YO between pulses of MIH release (Chang and Mykles, 2011). A cGMP-dependent protein kinase (PKG) is then activated and inhibits ecdysteroidogenesis through transcriptional and translational regulation (Lee et al., 2007; Chang and Mykles, 2011). Similarly, PKA which is activated in the 
triggering phase can also have a prolonged effect by directly inhibiting transcriptional and translational regulation of ecdysteroidogenic genes (Covi et al., 2012).

Intracellular regulation of cyclic nucleotide and calcium concentrations can also affect the sensitivity of the YO to MIH (Covi et al., 2008, Nakatsuji et al., 2009). A pronounced $\sim 2.5$ fold increase in phosphodiesterase (PDE) activity in the mid and late premolt stages is indicative of this reduction in YO sensitivity (Nakatsuji et al., 2006); especially considering that MIH binding to YO receptors is not reduced during premolt (Webster, 1993). Phosphodiesterases are involved in the degradation of second messenger cyclic nucleotides. The regulatory aspects of PDEs and other possible mediators of cyclic nucleotide ratios in the premolt $\mathrm{YO}$ is further supported by evidence that exposure of the committed $\mathrm{YO}$ to exogenous $\mathrm{MIH}$ has virtually no effect of ecdysteroidogenesis (Nakatsuji and Sonobe, 2004).

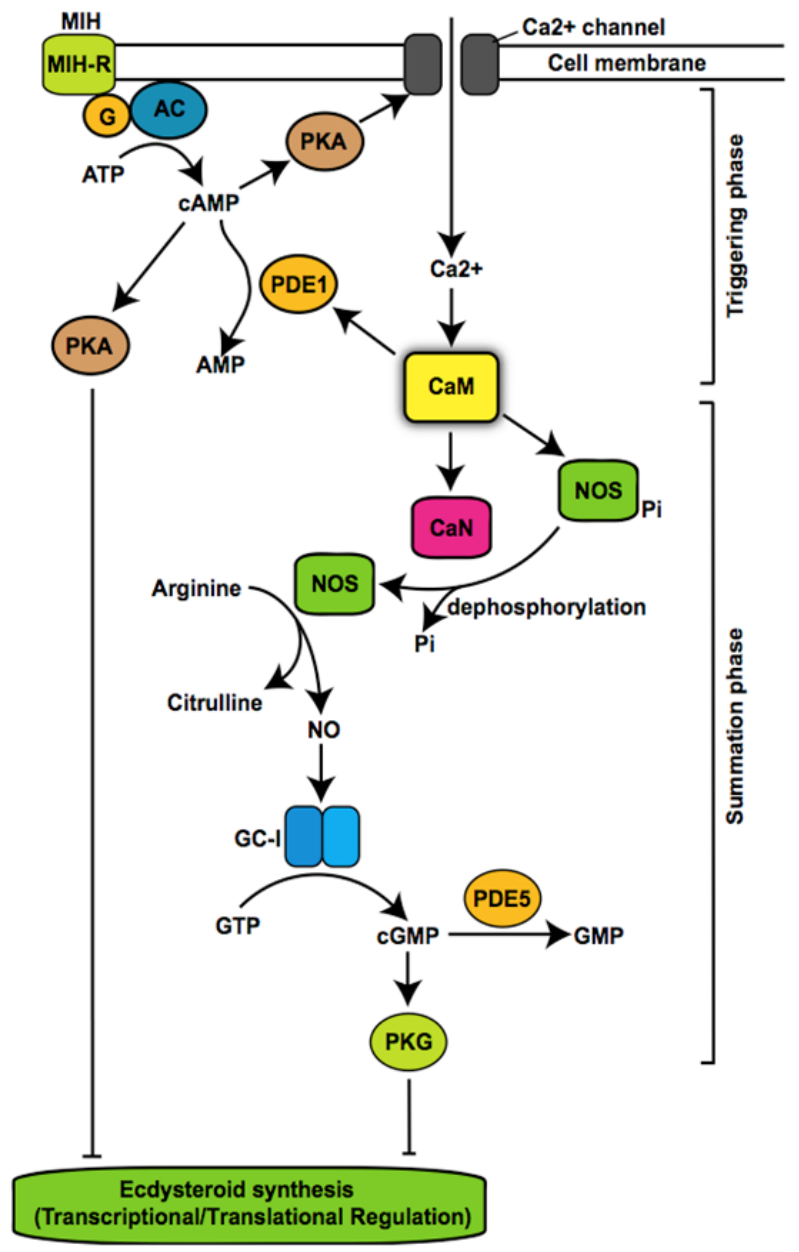

Figure 7. MIH binds to specific transmembrane receptors on cells of the YO resulting in a signaling cascade beginning with the conversion of ATP to cAMP by adenylyl cyclase. Increasing intracellular cAMP concentrations mediate protein kinase A (PKA) activity resulting in the opening of $\mathrm{Ca} 2+$ channels on the cell surface.

This leads to the activation of nitric oxide synthase (NOS) through calmodulin $(\mathrm{CaM})$ and calcineurin (CaN). Guanylyl cyclase-I (GC-1), which converts GTP to cGMP, is dependent on NO. As a result, protein kinase $G$ activity increased and inhibits ecdysteroidogenesis at the transcriptional and translational level. The major downstream transcriptional and translational regulator being inhibited is hypothesized to be the mTOR complex. (Adapted from Chang and Mykles, 2011). 
Regulation of the mechanistic target of rapamycin (mTOR) is one of the previously mentioned mechanisms for transcriptional and translational control of genes involved in ecdysteroidogenesis. mTOR activation may be specifically involved with the transition to the committed state, which is of particular interest as it pertains to MIH-insensitivity and crustacean growth. Identification of a myostatin (Mstn)-like factor cloned from G. lateralis indicates that activation of mTOR may be regulated through a putative transforming growth factor- $\beta$ (TGFß) superfamily pathway (Covi et al., 2008; Chang and Mykles, 2011). Further, transcript levels of mTOR and Akt, an activator of mTOR, were significantly increased in the premolt YO of $G$. lateralis (Abuhagr et al., 2016).

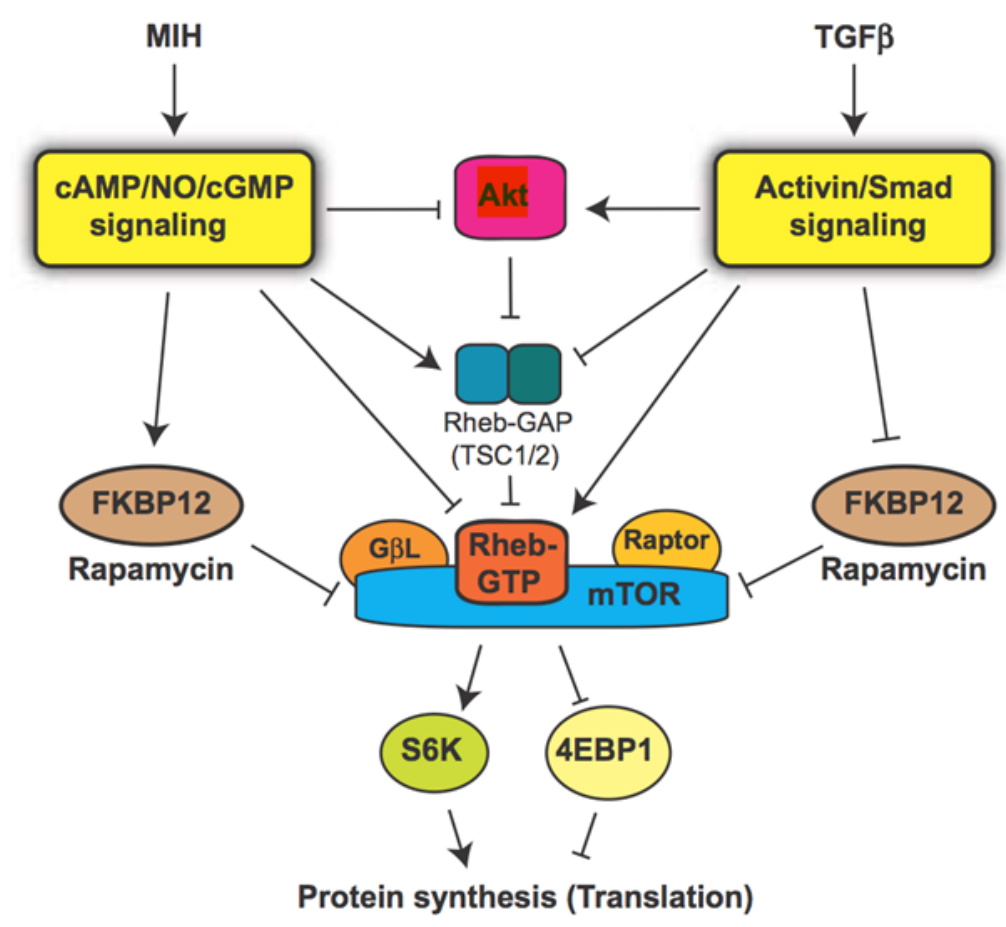

Figure 8. Proposed signaling mechanisms affecting the activation state of mTOR in the crustacean YO (Adapted from Abuhagr et al., 2016). 
The proposed coordination of YO-specific post-transcriptional mediation of the mTOR activation state is as follows (Fig. 8): MIH inhibition of mTOR activation occurs in the basal YO state of intermolt and postmolt crabs. Downstream signaling resulting from $\mathrm{MIH}$ receptor binding may interact with and activate FKBP12 (rapamycin) and Rheb-GAP which are potent inhibitors of mTOR. Specifically, Rheb-GAP inhibits mTOR via hydrolysis of Rheb-bound GTP. In its GTPbound state, Rheb associates with mTOR and phosphorylates S6K and 4E-BP1 which regulate translation of ecdysteroidogenic transcripts (Huang and Fingar, 2014; Inoki et al., 2003). Decreases in intracellular GTP via active GC-I may also provide an indirect means of inhibiting Rheb, and thus mTOR. Activation of the mTOR complex in the YO may occur through TGF $\beta$ activation of the Activin/Smad signaling pathway, in turn activating Akt which then inactivates Rheb-GAP (Abuhagr et al., 2014; Abuhagr et al., 2016).

Recent evidence provided by the proteomic analysis of the C. maenas molting gland has further suggested a mechanism for nutrient-sensing activation of mTOR in the premolt YO (Hamer, 2015). This hypothetical mechanism is further supported by a growing understanding of both environmental and cellular regulation of the mTOR signaling network. Specifically, increases in glucose may indirectly result in increases in intracellular $\alpha$-ketoglutarate $(\alpha-K G)$ which then promote mTOR translocation to lysosomes and activation of mTOR (Huang and Fingar, 2014). Increased abundance of glutamate dehydrogenase, the enzyme responsible for conversion of glutamine into $\alpha-\mathrm{KG}$, was found to be increased in abundance in the premolt $\mathrm{YO}$ of $C$. maenas (Hamer, 2015). A similar nutrient sensing activation of mTOR via increased intracellular amino acids, particularly leucine, has also been identified in mTOR. The necessary increase in available cellular energy and amino acids which ultimately result in ecdysteroidogenesis support this nutrient-sensing activation of the mTOR complex within the premolt YO. 
As is indicated by the generalized and somewhat brief description of the cellular mode of action of $\mathrm{MIH}$, and the activation of the YO upon entry into the premolt stages, there is still much to be learned about the cellular and molecular processes which govern the hormonal control of molting in crustaceans. Transcriptomic, metabolomic, and proteomic studies, such as the one presented here, can provide valuable and novel information regarding the cellular and physiological changes that command the molting process.

Once the YO has become activated, it then begins to synthesize and secrete ecdysteroids, which is as of our current knowledge, the sole function of the YO in crustaceans. Ecdysteroids are synthesized in the YO through conversion of cholesterol to ecdysone or similar ecdysteroid products (Mykles, 2011). Ecdysone is then secreted from the YO and transported through the hemolymph to peripheral tissues where it is converted to the functional molting ecdysteroid hormone, 20-hydroxyecdysone (20E). Crustaceans are unable to synthesize cholesterol de novo and therefore rely on dietary acquisition (Lachaise et al., 1993; Mykles, 2011). Another mechanism for MIH suppression of ecdysteroidogenesis, not previously mentioned, involves the direct inhibition of cholesterol uptake by cAMP (Covi et al., 2009). Ecdysteroid synthesis can be divided into two stages: (i) conversion of cholesterol to $5 \beta$-diketol and (ii) conversion of $5 \beta$-diketol to the secreted ecdysteroid products (Mykles, 2011). Both stages of ecdysteroid synthesis involve cytochrome p-450 mono-oxygenases encoded by the Halloween genes (Gilbert and Rewitz, 2009; Mykles, 2011). It is likely that MIH-induced transcriptional and translational inhibition of ecdysteroidogenesis involves the regulation of these genes.

The first stage of ecdysteroid synthesis (Fig. 9) is conserved among crustaceans and initially involves the conversion of cholesterol to 7-dehydrocholesterol (7DC) by a 7,8dehydrogenase encoded by the Halloween gene, Neverland $(N v d)$. The following steps are not well 
characterized and have therefore been labeled as the "black box" of ecdysteroidogenesis. The black box involves the conversion of 7DC to $\Delta^{4}$-diketol via the enzymes encoded by non-molting glossy (Nm-g)/Shroud (Sro), spook (Spo)/spookier (Spok), and spookiest (Spot). A 5ß[H]-reductase then reduces $\Delta^{4}$-diketol to $5 ß$-diketol, concluding the reactions involved in the first stage of ecdysteroid synthesis (Mykles, 2011).

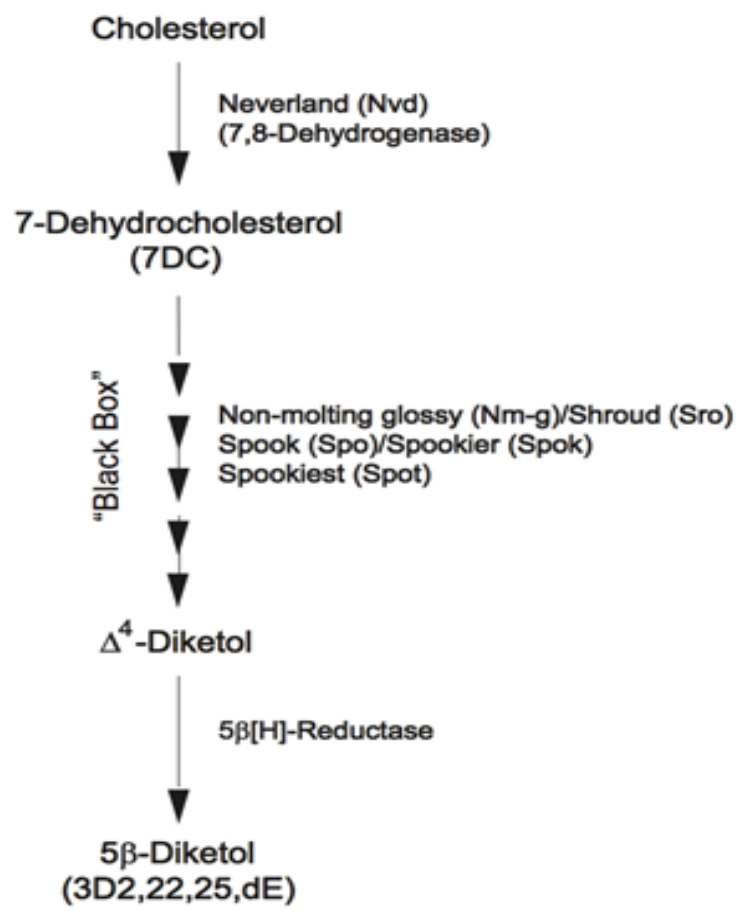

Figure 9. The first stage of ecdysteroid synthesis in the crustacean YO begins with the conversion of cholesterol to 7Dehydrocholesterol by a 7,8Dehydrogenase encoded by the Halloween gene, Neverland $(N v d)$. The following reactions are termed the "black box" and involve enzymes encoded by non-molting glossy/shroud, Spook/Spookier, and Spookiest. (Adapted from Mykles, 2011).

(3D2,22,25,dE)

Subsequent reactions involving the conversion of $5 \beta$-diketol to the secreted products are much better understood (Fig. 10). The pathway branches into 4 parts, each of which involve the Halloween genes phantom, disembodied, and shadow, but result in the secretion of 4 different products from the YO (Gilbert and Rewitz, 2009; Mykles, 2011). Conversion of 5ß-diketol to the secreted products primarily occurs within the inner membrane of the mitochondria (Hopkins, 2009). Secreted products from the YO into the hemolymph vary by species, developmental stage, molt stage, and season, and include 3-dehydro-25-deoxyecdysone (3D25dE), 3-dehydroecdysone 
(3DE), ecdysone (E), and 25-deoxyecdysone (25dE) (Mykles, 2011). Typically, no more than two different products are secreted from the YO of a given species at a given time. The formation of these secreted products involves a sequential hydroxylation of the core and side chains of cholesterol in such a manner that the process transpires in a directional manner (Lachaise et al., 1993). The most well accepted paradigm of steroid hormone excretion involves the diffusion of the lipophilic compounds directly across the cell membrane (Lachaise et al., 1993; Birkenbeil, 1983). However, cytological studies of the YO of several crustacean species have indicated that ecdysteroids might be excreted via vesicle-mediated exocytosis (Birkenbiel, 1983), a mechanism that will be discussed in more detail in Chapter II.

The final biosynthetic step of ecdysteroidogenesis occurs in the peripheral tissues after transport of the secreted products through the hemolymph (Fig. 10). The peripheral tissues involved include the gonads, hindgut, abdominal ganglia, eyestalk ganglia, hepatopancreas, antennal gland, and epidermis (Mykles, 2011). The final hydroxylation of carbon 20 occurs in the peripheral tissues via the 20-hydroxylase enzyme encoded by the Halloween gene, Shade (Mykles, 2011). The final active ecdysteroid products include ponasterone A (PA), 3-dehydro-20hydroxyecdysone (3D20E) and 20-hydroxyecdysone (20E), with 20E and PA being the major active ecdysteroid compounds present in crustacean hemolymph (Mykles, 2011). These final active ecdysteroid compounds are then circulated through the hemolymph where they initiate their proecdysial effects by binding to the ecdysteroid receptors that are present in nearly every organ and tissue (Spindler et al., 2009). The most well characterized effects of 20E and related ecdysteroid compounds includes the reabsorption and synthesis of a new exoskeleton, regeneration of lost limbs, and the degradation of claw muscle, all of which have been previously described in this chapter. However, the presence of ecdysteroid receptors on the multitude of other organs and 
tissues is indicative of a more wide-spread proecdysial process that has yet to be characterized. Interestingly, a negative feedback mechanism within the YO itself may result from high concentrations of circulating hemolymph ecdysteroids, ultimately resulting in the transition of the YO from the committed to the repressed state (Kim et al., 2005).

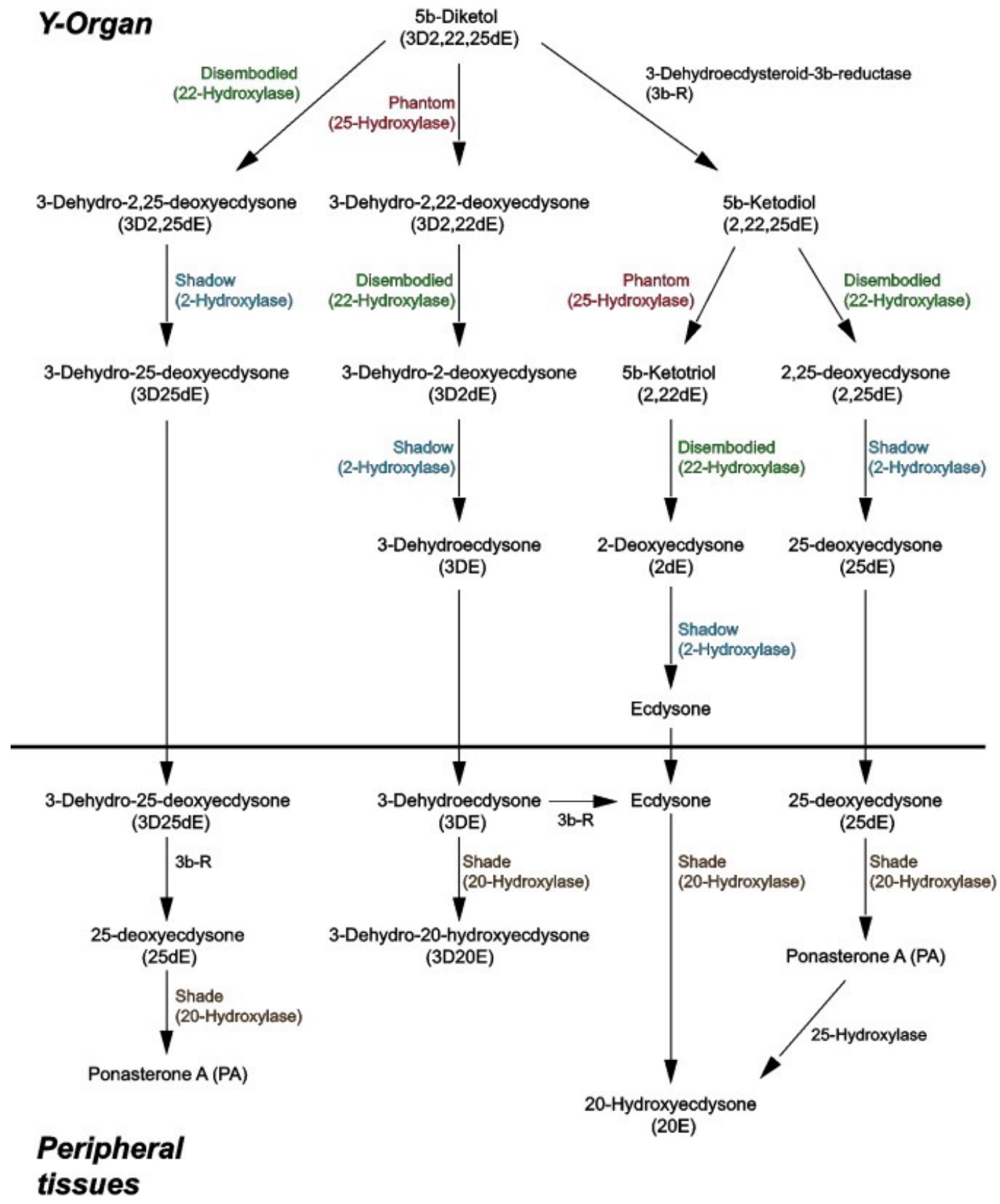

Figure 10. The second stage of ecdysteroid synthesis occurs in the Y-organ and the peripheral tissues and involves enzymes encoded by the Halloween genes, Disembodied, Phantom, Shadow, and Shade. (Adapted from Mykles, 2011). 
Catabolism of ecdysteroids into highly polar conjugates can occur in various tissues, although excretion of these polar conjugates primarily occurs through the antennal gland (Mykles, 2011). Changes in the rate of conversion of ecdysteroids to their inactive metabolites by the antennal gland can determine the circulating hemolymph ecdysteroid titers throughout the molt cycle, particularly the precipitous drop which occurs in late premolt (Mykles, 2011). What is not excreted by the antennal gland is broken down into apolar conjugates by the hepatopancreas and excreted in the feces (Mykles, 2011). The circulating levels of ecdysteroids throughout the molt cycle are ultimately balanced between changes in rates of synthesis and excretion.

As laid out in this section, the YO is a complex organ, the function of which involves several levels of regulation from environment, to life history, to molecular. As such, further studies are needed to fully describe the aspects of cellular and molecular regulation that remain elusive. The importance of understanding all levels of regulation of crustacean molting processes will be described briefly in the following section.

\section{Socioeconomic Impacts and Relevance of Molting in Crustaceans}

Arthropoda is such a diverse and prevalent phylum of animals that they comprise an estimated $80 \%$ of all known and living animal species (Ødegaard, 2000). The subphylum of Crustacea alone includes an approximate 67,000 described species (Zhang, 2011). Given the globally widespread presence of arthropods it should come as no surprise that their success greatly

impacts areas from ecosystem health to food security and the global economy. The highly conserved crustacean molting mechanisms previously described are also similar to the molting process of other arthropods such as in insects, involving many of the same regulatory molecules and processes. 
Aquaculture is currently the fastest growing food-producing sector in the world. Globally, the crustacean aquaculture industry is worth more than US\$10 billion (Stentiford et al., 2012). In North and South America alone crustacean aquaculture comprises $21.7 \%$ of the total volume of fisheries aquaculture (FAO, 2016). Penaeid shrimp production dominates the current market of farmed crustaceans, followed closely by the giant freshwater prawn, Macrobrachium rosenbergii. Prevalent viral and bacterial zoosanitary (infecting the animal but not the consumer) infections are the primary source of difficulties with production in the crustacean aquaculture sector (Stentiford et al., 2012). Based on estimates from 2012 up to $40 \%$ of tropical shrimp production is lost annually due to pathogenic disease (Stentiford et al., 2012).

The health of natural fisheries is also threatened, with a reported spread of disease occurrence in wild populations increasing, presumably in associating with changes in climactic conditions. Given the global importance of the crustacean aquaculture industry (FAO, 2016), much of our current knowledge of viral pathogens of crustaceans is based on the predominant farmed species, representing a tiny fraction of crustaceans as a whole. While disease and infection in farmed and wild crustaceans may seem to be distinct from our understanding of the molting process on the surface, the two are in fact highly correlated. Upon ecdysis, the soft-exoskeleton of the organism is now exposed to the environment, leaving the organism highly susceptible to infection by a variety of hosts. Data presented in Chapter II suggests an increased immune response within the YO upon activation. These data may not only reflect a possible immune role of the crustacean YO, as was recently suggested of the prothoracic gland of the insect (Stanley, 2006), but are also reflective of a correlation between the timing of growth and infection in crustaceans (Bateman et al., 2011). 
Given the current status of wild and farmed crustacean as a global source of food security alongside predictions of an expansion of the human population to between 8.8 and 9.1 billion by 2050 (FAO, 2016; Anderson, 2010), it is more important now than ever to understand all aspects of crustacean physiology. Current research is being applied to increase growth rates of farmed crustacean species in an attempt to meet ever growing production demands. For example, competitive inhibitors of MIH binding to receptors within the YO are being developed by pharmaceutical companies to target the growth of crustacean livestock (Shrivastava and Princy, 2014). Similar methods may be applied to inhibit growth of certain parasitic crustaceans, such as the common sea louse, which affect wild and aquaculture fisheries.

Beyond their role as a protein source for humans, our understanding of crustacean growth has the potential to directly affect human growth. Synthesis and degradation of somatic muscle in the chelae of crustaceans over the molt cycle is under regulatory control of ecdysteroids (Skinner, 1966). Developing our understanding of the signaling pathways which regulate protein synthesis and degradation in the muscle tissue have the potential to translate to homologous pathways within human tissue and can play a role in our understanding of certain diseases associated with muscular atrophy.

Finally, crustaceans are important contributors to ecosystems ranging from coastal dunes to the open ocean. They can regulate recruitment of fouling species to bays and estuaries, plant species in coastal regions, as well as higher-trophic level species which rely on crustaceans as a food source from cetaceans to sea otters. Numerous crustacean species have established invasive populations globally, including the Chinese mitten crab, Eriocheir sinensis, and the green shore crab, Carcinus maenas (Roman and Darling, 2007). These species compete with native crustacean species, having great impacts on trophic systems. Global climate change is affecting marine 
populations at alarming rates, exerting effects on aspects such as survival, spread of pathogens, growth rates, and timing of reproduction to name a few (Tomanek, 2014). Given the diverse roles of crustaceans which affect humans both directly and indirectly, it is of great importance to understand all aspects of their being, from the molecular regulation of molting to the population dynamics of invasion.

\section{Proteomics}

Proteomics is a relatively new term used in the field of molecular biology to describe the functions of specific cell types, tissues, or organs by the expression or modification of specific proteins (Lovríc, 2011). Proteomics is one of many "omics", or fields of study which aim to characterize and quantify biological molecules in relation to their overall function in a given organism. Aside from proteomics, the main fields of study include genomics, transcriptomics, and metabolomics, although new "omic" fields are continuing to emerge.

\section{Proteomics as a Hypothesis-Generating Tool}

Ultimately, these new fields stem from the foundation laid by the study of genomics. Although the structure of DNA was not characterized until 1952 (Watson and Crick, 1953), studies of the genome began as early as 1869 when the Swiss physician and scientist Friedrich Miescher first observed DNA in the nuclei of cells left behind on used bandages (Dahm, 2008). The Human Genome Project launched in 1990 by the US government provided the first major genomic study, the results of which were expected to provide answers for some of the most complex biological questions (Collins et al., 2003). As we are well aware of today, however, the mapping of the human genome created more questions than it answered. Thus the "omics" fields emerged, seeking answers to complex biological questions about the mechanisms that control functional processes of cells, tissues, organs, and organisms as a whole. The "omics" themselves, although often 
achieved as individual experiments, are synergistic. That is, changes in one area such as the transcriptome may help to explain observed changes in another area, such as the proteome, and vice versa.

Proteomic techniques are specifically useful in determining the cellular changes that occur over time or when the organism is exposed to a specific set of stressors. The Environmental Proteomics Laboratory at Cal Poly (San Luis Obispo, CA, USA) has been studying the effects of acute and chronic exposure to various environmental stressors such as high temperature, low oxygen, and changing salinities on marine organisms since 2005 (Tomanek, 2014). These studies have helped to elucidate the critical cellular responses marine organisms use when adapting to their changing environments.

This study provides functionally relevant data into naturally occurring developmental processes of crustaceans. Molt stages themselves are characterized by distinct physiological changes within the YO, and analysis of protein expression can lead to a comprehensive analysis of cellular-level functions and status of the molting gland. The proteomic analysis presented in Chapter II has yielded information about the energy demand, stress response, immune response, and homeostatic mechanisms involved with the activation, commitment, and repression of the crustacean Y). Used in tandem with genomic and transcriptomic datasets being developed by collaborators (Dr. Donald Mykles of Colorado State University and Dr. David Durica of The University of Oklahoma), this study will help to resolve the various levels of organization and regulation involved with changes in the YO over the course of a molt cycle.

The following sections will focus on detailing the general proteomic workflow and its specific application in assessing changes in the YO over the course of the molt cycle. Briefly, there are no hard-and-fast protocols through which proteomic analyses are performed. Rather, all 
proteomic analyses include the major components: (i) protein isolation and quantification, (ii) protein separation and analysis, and (iii) protein identification (Lovríc, 2011). As such, the described protocol will focus on the methodology specific to this experiment which use 2dimensional gel electrophoresis and matrix-assisted laser desorption/ionization tandem time-offlight (MALDI ToF/ToF) mass spectrometry. Special considerations and challenges in proteomic analyses will be noted throughout the following sections as relevant.

\section{Proteomic Analysis: Protein Isolation and Quantification}

Initial sample preparation and isolation is extremely important in proteomic studies, as this step will dramatically affect the type and amount of proteins identified later in the study. It is important to consider the cellular location of proteins when determining how to isolate them, as some proteins such as those embedded in the membrane require different reagents and procedures than cytosolic proteins (Lovríc, 2011). Further, preservation of post-translational modifications (PTMs) is undeniably important, as certain PTMs change the function or activation state of proteins. Certain protocols involve reactions that can lead to chemical modifications, such as oxidation, of proteins or their associated PTMs. Finally, the techniques used in sample preparation should leave the sample composition compatible with subsequent procedures, such as those used for separation and identification. Given all of these considerations it is reasonable to say that protein isolation can be more of an art form than a scientific procedure. Technically speaking, the true definition of the proteome encompasses all proteins expressed in a cell at any given time. However, the extreme variability in terms of the protocols needed to isolate specific proteins or PTMs creates the need for a seemingly endless sample in order to capture the entire proteome. The design of a given protein isolation protocol, therefore, should be tailored in accordance with the objective of the proteomic analysis. 
Following sample dissection, the first step in protein isolation is cell disruption which is needed to release intracellular proteins. This is typically accomplished through mechanical homogenization of the sample in a lysis buffer. The lysis buffer used typically is designed to not only aid in cell disruption, but also accomplish the necessary tasks of denaturing proteins in order to minimize protein aggregation as well as to inhibit endogenous proteases from degrading the protein sample (López, 2007). The chaotropic agents urea and thiourea are used in lysis buffers in order to disrupt hydrogen bonds and hydrophobic interactions, aiding in the denaturation of secondary and tertiary protein structure (Shaw and Riederer, 2003). The reducing agent dithiothreitol (DTT), also present in lysis buffer, serves to maintain proteins in their unfolded state, primarily through maintaining disulfide bonds in their reduced state. Membrane proteins are disrupted and solubilized through the use of a zwitterionic detergent, ASB-14 (Lovríc, 2011). Finally, a Tris-base buffer and carrier ampholytes (IPG buffer) maintains solubility and allows for efficient separation of proteins. Specific $\mathrm{pH}$ ranges of IPG buffer can be used to reduce the challenges of separating highly acidic or alkaline proteins, and aid in the separation of only those physiologically relevant proteins (Görg et al., 2009). In this study, samples between $\mathrm{pH}$ 3-10 were initially isolated and separated, however an extremely high concentration of proteins around $\mathrm{pH} 6$ made it difficult to separate individual proteins. Thus, we switched to focusing on proteins between pH 5-8 by changing the IPG buffer used in the sample.

Once full homogenization of the tissue is complete and no insoluble fractions remain, the non-protein cellular components (lipids, carbohydrates, nucleic acids, and salts) must be removed. Subcellular fractionation is accomplished through centrifugation, separating the cellular components based on their density. Following a series of centrifugation steps, trichloroacetic acid (TCA) and acetone are used to precipitate the protein and remove any remaining contaminants; 
however, some loss of protein typically occurs during this process (Görg et al., 2004). These purification steps are particularly important as the presence of nucleic acids, salts, or lipids can greatly effect subsequent protein identification. Nucleic acids are negatively charged and thus can interfere with isoelectric focusing when bound to proteins. Salts can also interfere with isoelectric focusing by precipitating proteins out of the IEF strips and increasing electrical conduction used during the $1^{\text {st }}$ dimension of separation. Finally, lipids can hydrophobically bind to proteins, affecting their solubility in the gels as well as the charge of the protein (Shaw and Reiderer, 2003).

The TCA/acetone precipitation steps leave the proteins dehydrated, so subsequent rehydration in a salt-free buffer is needed to prepare the sample for the first dimension of separation. Rehydration buffer contains chaotropes and ampholytes as were present in the homogenization buffer, but also contains octylphenoxypolyethoxyethanol (Nonidet P-40), a detergent that solubilizes membrane proteins and prevents protein aggregation (Lovríc, 2011). CHAPS (3-[(3-cholamidopropyl) dimethylammonio]-1-propanesulfonate) and dithioerythritol (DTE) are additional zwitterionic and reducing agents, respectively, used to maintain solubility of proteins and prevent reformation of disulfide bonds (Lovríc, 2011).

Following the isolation steps, the amount of protein present in each sample must be quantified before separation can occur. Protein quantification is necessary in order to ensure that the same amount of protein (e.g. $400 \mu \mathrm{g}$ ) is loaded for each gel, preventing confounding effects of differences in total protein content in subsequent analyses. The 2-D Quant Kit (GE Healthcare Bio-Sciences) is used to determine protein concentrations in each sample. The kit uses the Lowry method of protein quantification in which protein-catalyzed reduction of $\mathrm{Cu}^{2+}$ to $\mathrm{Cu}^{+}$is quantified using a spectrophotometer (Weist et al., 2008; 2-D Quant Kit, GE Healthcare). The Bradford assay is another common method of determining protein concentration, but is typically not used in 
proteomic studies as it is sensitive to the presence of detergents and reducing agents that are present in the buffers previously described (Bradford, 1976). Once isolation and quantification of the proteins is complete, the samples are ready for separation and subsequent analysis.

\section{Proteomic Analysis: Protein Separation and Analysis}

Isoelectric focusing (IEF) is the first step in the protein separation procedure, and serves to separate the proteins based on their isoelectric point (pI), or the $\mathrm{pH}$ at which a proteins charge is neutralized. IEF takes advantage of the intrinsic charge of amino acid R-groups. Polyacrylamide gels containing acrylamide monomers with attached R-groups are used to create an immobilized $\mathrm{pH}$ gradient (pH 5-8 in this study) along a strip (IPG strip). The protein sample is then solubilized into the gel and an electrical current is applied to both ends of the IPG strip. Proteins then migrate through the gel until settling in the region of the gradient at which the charges of the R-groups contained in the protein are neutralized (Görg et al., 2009; Lovríc, 2011). A series of charges is used in order to ensure all of the proteins within the sample have completely migrated based on their intrinsic pI. Upon completion of isoelectric focusing, the IPG strips are immediately frozen at $-80{ }^{\circ} \mathrm{C}$ to ensure stability of the proteins within the polyacrylamide gel.

The second dimension of separation uses sodium dodecyl sulfate polyacrylamide gel electrophoresis (SDS-PAGE) to separate proteins by their molecular mass. In preparation for SDSPAGE, IPG strips are first incubated in the rehydration buffer containing DTT and a Tris-base buffer ( $\mathrm{pH} 8.8$ ). A second equilibration step follows, in which the strips are incubated in the same buffer except with the compound iodoacetamide (IAA) rather than DTT. IAA permanently prevents disulfide bridges from reforming by binding with DTT and cysteine residues (Görg et al., 2009). As with other methods of gel electrophoresis, altering the percentage of acrylamide and bisacrylamide within the gel can be used to separate higher or lower molecular weight proteins as 
desired (Lovríc, 2011; Berth et al., 2007). In this study proteins between 14.4 to $97.4 \mathrm{kDa}$ were targeted. Equilibrated IPG strips are placed on top of the polyacrylamide gels and an electric current is applied which forces the proteins to migrate through the gel matrix based on their molecular weight, with smaller proteins migrating faster than larger proteins.

Following second dimension electrophoresis gels are stained so that the proteins may be visualized for analysis. Depending on the amount of proteins in the gels and the type of identification procedure that will be used, different staining methods are available. Fluorescent dyes such as SYPRO Ruby can be used, or colorimetric stains, such as or Colloidal Coomassie Blue (CCB), which can be visualized without the use of UV light (Berth et al., 2007). CCB is commonly used because its binding is not only reversible, making destaining easy, but it also fixes the proteins within the gels so that they may be stored in a cold environment for a few weeks without degrading (Berth et al., 2007). One drawback of CCB is that it requires a fair amount of protein to bind, so proteins with lower abundance may be missed (Lovríc, 2011). Silver staining is the most sensitive of the colorimetric stains; however it is incompatible with the ionization mechanism used during mass spectrometry (Görg et al., 2009).

Analysis of protein abundance utilizes digitized images of the stained gels. Software packages are used for gel image analysis, such as Delta 2D (Decodon, Greifswald, Germany). Due to minor differences in samples, temperature, $\mathrm{pH}$, and inherent proteomic differences that cause slight variation in the migration of proteins through the gels, the ability to superimpose, or "warp" protein spots is a vital component of these image analysis programs (Berth et al., 2007; Lovríc, 2011). Warping of the gel images allows for corresponding protein spots on each gel in the experiment to be aligned so that they can be quantified accordingly. Warping can be completed manually or automatically, depending on the program, using match vectors that connect 
corresponding protein spots based on patterns within each gel image (Lovríc, 2011). Gel images for experimental replicates or treatment groups are first warped together, then a "fused-gel" composite image is created based on the normalized pixel density for each individual protein spot (Berth et al., 2007). Normalization of the pixel density accounts for inherent differences between gel images that may be due to protein loss during IEF or SDS-PAGE, or differences in staining efficiency (Berth et al., 2007). The spot pattern generated by this composite image can then be used to statistically compare relative protein expression for each spot among samples and treatments (Berth et al., 2007; Lovríc, 2011). Statistical analysis in Delta 2D is accomplished using "spot volume" which is a function of spot boundaries and pixel density and serves as a proxy for relative protein abundance (Berth et al., 2007). Thus, changes in the spot volume of an individual protein spot among treatments correspond with changes in expression of that protein.

Changes in protein expression across treatment groups are often statistically visualized using principal component analysis, heat maps, and expression profiles. Principal component analysis is a multivariate statistical approach which is used to visually represent which treatment groups are more or less similar in expression patterns for all detected proteins (Ringnèr, 2008). Heat maps are used to display abundance patterns for individual proteins across each gel image, or normalized for each treatment. Using Pearson correlation, heat maps can be structured so that proteins with similar abundance profiles are displayed next to each other. Expression profiles are created for individual protein spots, and post-hoc analyses can be used to determine statistical difference among individual samples or treatments. There are numerous statistical analyses that can be used to interpret proteomic datasets. Based on the desired use of the proteomic data for an experiment, the statistical approach to be used should be considered before the experiment begins (Berth et al., 2007). 


\section{Proteomic Analysis: Protein Identification}

The final step in proteomic analysis is protein identification. As previously mentioned, sample preparation and separation steps can greatly affect which identification strategies can be employed. The 2D gel based approach was used in this experiment as samples from this approach can be prepared for MALDI-ToF/ToF quite easily (Lovríc, 2011). Other methods of protein analysis can be used for samples that are not separated on a gel, but instead are separated through liquid chromatography (Lovríc, 2011).

Mass spectrometry (MS) relies on the ionization of analytes to measure the mass-to-charge ratio $(\mathrm{m} / \mathrm{z})$ and the number of ions detected for a given $\mathrm{m} / \mathrm{z}$. This generates a list or series of fragmented masses of the protein. Subsequent identification of that protein relies on the comparison of those fragmented masses to genomic or transcriptomic databases (Lovríc, 2011). The MALDI-ToF/ToF method of mass spectrometry uses a laser to charge tryptic fragments of proteins, while electrospray ionization (ESI) ionizes peptides in solution and is thus the method of choice for mixtures of proteins using high performance liquid chromatography [HPLC (Lovríc, 2011)].

To prepare samples for MALDI-ToF/ToF analysis, individual proteins must first be carefully excised from the 2D gels. Digestion with specific proteases then cleaves proteins at known locations so that predicted sequences and $\mathrm{m} / \mathrm{z}$ can be calculated from the genomic or transcriptomic databases. Trypsin is the most commonly used protease because it is inexpensive, easy to isolate, has high specificity, and works over a range of concentrations and conditions (Lovríc, 2011). Trypsin cleaves at the C-terminal of lysine and arginine residues, and leaves a mixture of peptide fragments digested from the protein within the excised gel. Digested peptide fragments are then co-crystallized within a matrix on a target plate (Lovríc, 2011). Hundreds of 
proteins can be loaded onto individual anchors on a target plate for mass-analysis of the proteome. The matrix itself serves to protect the peptides from degradation as well as confer a positive charge to the fragments so that they are accelerated towards the mass analyzer by voltage plates upon ionization by the laser (Lovríc, 2011). When the laser is fired at a specific anchor on the target plate, the matrix and peptides are vaporized into a gaseous state.

The mass analyzer measures the $\mathrm{m} / \mathrm{z}$ ratio of the ionized peptides after they have accelerated through the vacuum. A linear time-of-flight (TOF) mass analyzer is typically used with the MALDI method of ionization (Lovríc, 2011). TOF analyzers calculate $\mathrm{m} / \mathrm{z}$ based on the premise that peptide fragments carrying the same charge will travel through the vacuum at different speeds based on their mass. Unique spectral outputs called peptide mass fingerprints (PMF) are created for each protein based on the $\mathrm{m} / \mathrm{z}$ ratio of each ionized peptide fragment (Lovríc, 2011). Protein identification occurs by comparing PMFs from each sample to theoretical PMFs for proteins based on known tryptic sites within their sequence (derived from the genomic or transcriptomic databases). Peptide fragment fingerprints (PFFs) are generated through further fragmentation of PMFs (Lovríc, 2011). MALDI-ToF/ToF mass spectrometers include two ToF analyzers, as indicated by the name, separated by a collision cell. A single peak representing a high abundance peptide of a single $\mathrm{m} / \mathrm{z}$ ratio is selected and then fragmented using a collision induced dissociation (CID) chamber. The CID contains an inert gas, such as argon, in order to further fragment the peptide before reaching another detector that allows for determination of the amino acid sequence of the selected PFF based on its secondary $\mathrm{m} / \mathrm{z}$ and time-of-flight through the vacuum (Lovríc, 2011). This type of tandem mass spectrometry (MS/MS) increases the accuracy of protein identification as it is extremely unlikely that two different proteins would share both a PMF and a PFF (Lovríc, 2011). 
As previously described, experimental data (PMFs and PFFs) for individual proteins are compared against theoretical PMFs and PFFs based on predicted locations of tryptic cleavage within a given protein sequence. Protein or expressed sequence tag (EST) databases are searched using a program called MASCOT (Matrix Science, Inc., Boston, MA, USA). Mascot calculates the statistical probability of peptide matches between the identified spectra and the searched sequence databases based on the resolution of each spectra.

A molecular weight search engine (MOWSE) score is assigned to each identified protein based on the probability that the protein identification match occurred by chance alone. Higher MOWSE scores are more likely to be deemed significant, or a non-random match to a given protein ID. A minimum cutoff of at least two matched PFFs is used for each protein ID in order to ensure accurate protein identification (Tomanek and Zuzow, 2010). Because the primary EST library for G. lateralis used in this study was not yet fully annotated at the time of protein identification, sequence matches were then run through NCBI Basic Local Alignment Search Tool (BLAST) to identify homologous proteins with previously annotated sequences.

Following the completion of protein identification, the results of a more-or-less comprehensive proteomic dataset can now be interpreted. Consideration of the tissue in context of the whole organism biological system is important when interpreting proteomic data. Although reductionist in the proteomic experimental approach, it is important to "put humpty-dumpty back together" by considering protein abundance changes in terms of broad functional categories and their relevance to the physiology of the whole organism (Tomanek, 2014). 


\title{
II. MANUSCRIPT
}

\begin{abstract}
Molting in crustaceans is a highly complex physiological process involving negative regulation by two paired endocrine glands, the X-organ/sinus gland complex (XO/SG) and the Y-organ (YO).
\end{abstract} The $\mathrm{XO} / \mathrm{SG}$ complex is responsible for making molt-inhibiting hormone (MIH) which negatively regulates synthesis of the molting hormones, ecdysteroids, by the YO. Analysis of gene expression in the XOs and YOs has led to the development of a proposed molecular signaling pathway which regulates ecdysteroidogenesis and subsequent molting in crustaceans. In this study, changes in protein abundance in the $\mathrm{YO}$ were characterized over the course of a molt cycle (intermolt, early premolt, mid premolt, and late premolt) induced by multiple leg autotomy (MLA) in the blackback land crab, Gecarcinus lateralis. In all, 457 distinct protein spots were detected in the molting gland using two-dimensional gel electrophoresis, of which $230(50 \%)$ changed significantly in abundance over the course of the molt cycle (one-way permutation ANOVA, $\mathrm{p} \leq 0.05$ ). Changes in protein abundance were most notable between the intermolt and the three premolt stages, indicative of a biological 'on-off' switch in the Y-organ. Several hemolymph species proteins, including hemocyanin, cryptocyanin, and transglutaminase, were identified and changed abundance due to functions associated with organs and tissues other than the Y-organ. An abundance of cytoskeletal proteins were identified which correspond with glandular hypertrophy and are indicative of vesicular-mediated exocytosis, possibly of ecdysteroids. Further, several proteins involved in the immune, proteostasis, and oxidative stress response are characteristic of supporting the dynamic and demanding cellular changes, i.e., protein synthesis, associated with ecdysteroidogenesis and the transition of the Y-organ from the basal to the highly active state. Many proteins involved in energetic pathways including glycolysis, the citric acid cycle, amino 
acid metabolism, and one-carbon metabolism changed in abundance in response higher energy demands and greater requirement of the biosynthetic precursors of the YO over the molt cycle. Taken together, these changes in diverse physiological pathways represent the complexity involved with regulation of the Y-organ, even with just the single proposed physiological purpose of ecdysteroidogenesis.

Key Words: Gecarcinus lateralis, molting, Y-organ, energy metabolism, ecdysteroids 


\section{INTRODUCTION}

Growth in arthropods is a complex process that is restricted by a hardened exoskeleton. Initially, the animal must synthesize a new layer of cuticle underneath the hardened carapace, then loosen the connective tissue between this newly synthesized layer and the old exoskeleton. During ecdysis, or molting, the carapace is split on the posterior side and the animal removes itself from the confines of the old exoskeleton using peristaltic motion (Chang and Mykles, 2011). Upon release from the old exoskeleton, the organism must ingest large amounts of air or water to expand the new exoskeleton while it is still flexible, then rapidly harden the exocuticle for locomotion and protection from predators.

More than 10 million metric tons of farmed and captured crustacean catch is sold on the global market annually. The aquaculture industry is currently dominated by farming of marine shrimp, the fastest growing sector (Stentiford et al., 2012). With the ever increasing demand for seafood alongside rapidly declining fisheries it is vital to take measures to ensure safe products, not only for human consumption but also for the health of fisheries. Studies regarding the molecular regulation of molting may introduce new methods to manipulate molting and produce rapidly growing crustacean seafood products to meet increasing industry demands.

Molting in crustaceans is a cyclical process controlled by two sets of paired endocrine organs, the $\mathrm{X}$-organ/sinus gland complex $(\mathrm{XO} / \mathrm{SG})$ in the eyestalks, and the molting gland known as the $\mathrm{Y}$-organ $(\mathrm{YO})$ in the cephalothorax. Synthesis of the pro-molting hormone, ecdysteroid, by the YO is negatively regulated through secretion of the neuropeptide molt-inhibiting hormone (MIH) by the XO/SG (Webster, 1986). Throughout the molting process the YO transitions through four physiological stages: the "basal" state at postmolt and intermolt; "activated" state at early premolt; "committed" state at mid premolt; and "repressed" state at late premolt and early postmolt 
(Chang and Mykles, 2011). Over the course of a natural molting cycle, hemolymph ecdysteroid titers can be used to monitor the state of the YO.

YO physiological states are mediated by endocrine and paracrine control. Decreased synthesis and secretion of MIH triggers the transition from the basal state in intermolt $\left(\mathrm{C}_{4}\right)$ to the early premolt $\left(\mathrm{D}_{0}\right)$ activated state, upon which the YO hypertrophies and ecdysteroid synthetic capacity increases. YO activation requires mTOR activity (Abuhagr et al, 2014, 2016). A putative Transforming Growth Factor-beta (TGF- $\beta$ ) triggers the transition of the YO into the mid premolt $\left(D_{1,2}\right)$ committed state and becomes refractory to MIH (Abuhagr et al., 2016). A subsequent rise in ecdysteroid titers elicits the transition from the committed state to the repressed state in late premolt $\left(\mathrm{D}_{3,4}\right)$, after which hemolymph ecdysteroid titers fall and initiate ecdysis. The YO atrophies as it returns to the basal state during late postmolt (stage $\mathrm{C}_{1-3}$ ) and,sensitivity to $\mathrm{MIH}$ returns and hemolymph ecdysteroid titers remain at a basal level (Chang and Mykles, 2011).

The proposed mechanism of MIH inhibition in the YO involves two phases: (i) the 'triggering' phase involves MIH binding to a G-protein coupled receptor and subsequent activation of adenylyl cyclase (AC), and (ii) 'summation' includes downstream activation of nitric oxide synthase (NOS). Nitric oxide appears to be an important second messenger in MIH signaling mechanisms by activating guanylyl cyclase within the YO, which leads to an increase in intracellular cGMP and downstream inhibition of ecdysteroidogenesis (Chang and Mykles, 2011). Ecdysteroidogenesis, on the other hand, appears to be largely mediated by a protein complex called "mechanistic target of rapamycin" (mTOR), which is responsible for controlling the translation of over 800 mRNA products into protein (Abuhagr et al., 2016). The committed YO in mid premolt is characterized by insensitivity to $\mathrm{MIH}$, likely through the inhibition of MIH signaling 
components by increased translation of yet to be characterized "commitment genes" (Chang and Mykles, 2011).

Experimental manipulation of molting in the blackback land crab, Gecarcinus lateralis, provides a straightforward and fairly quick means of studying molt-related processes. Molting can be induced through two different methods: (i) Multiple-leg autotomy (MLA) involves the removal of five or more walking legs from the organism. Following MLA, limb buds, or primary regenerates of the walking legs, begin to form and the molt stage can be calculated by the ratio of limb bud size to carapace length, or R-index (Chang and Mykles, 2011). (ii) Eyestalk ablation (ESA) entails the removal of both eyestalks, thus eliminating the source of molt-inhibiting hormone.

Proteomic analysis using 2-dimensional gel electrophoresis alongside matrix-assisted laser desorption tandem time of flight (MALDI-ToF/ToF) is an invaluable method for elucidating the physiological changes in crustaceans over the course of a molt cycle. This method is discoverybased, rather than hypothesis-driven and can lead to invaluable insight and further research opportunities, particularly when utilized alongside transcriptomic studies. Previous proteomic analysis has detected changes in metabolic, cytoskeletal, and stress response proteins over the course of the molt cycle in the green shore crab, Carcinus maenas (Hamer, 2015).

This study assessed proteomic level changes in the molting gland of the blackback land crab, Gecarcinus lateralis, over the course of a molt cycle induced by multiple leg autotomy (MLA). Future analysis of the data presented within this study will be used in conjunction with the transcriptomic library of the G. lateralis YO (Das et al., 2016) to further elucidate the molecular-level mechanisms controlling molting within crustaceans. 
Paired molting glands were harvested from a total of 36 crabs representing the intermolt, early premolt, mid premolt, and late premolt stages. Biological replicates of each molting stage were composed of three paired YOs due to the small size, and thus protein abundance, of the individual glands, with similar hemolymph ecdysteroid titers. Protein abundances were visually and statistically compared on two-dimensional gels, and proteins were identified using MALDIToF/ToF tandem mass spectrometry.

Our analysis revealed discrete changes in protein abundance across the molt cycle. Distinct differences in protein expression between intermolt and the three premolt stages suggest a biological 'on-off' switch between the basal and activated Y-organ. Further, slight changes in protein abundances within the early, mid, and late premolt Y-organ revealed changes in the function of the gland as the molt cycle progressed. Owing to the inherent complexity of metabolic pathways, elucidating a single answer to the question of molt cycle regulation may be an inexhaustible task. Our data supports a multifaceted mechanism involving regulation of energy metabolism, immune response, proteostasis, cytoskeleton, and reactive-oxygen scavenging within the molting gland, by which changes in protein abundance and post-translational modifications allow for the vital functions provided by the YO. 


\section{MATERIALS AND METHODS}

\section{Animal Collection and Husbandry}

Adult male Gecarcinus lateralis were collected during May 2013 and 2014 from the Dominican Republic and shipped via commercial airline to Colorado State University. Crabs were housed in communal (7-12 animals per cage) plastic cages lined with Aspen bedding moistened with 5 p.p.t. Instant Ocean (Aquarium Systems, Mentor, OH, USA). Temperature of the room was kept at $22^{\circ} \mathrm{C}$ and relative humidity was maintained between $75-95 \%$, along with a $12 \mathrm{~h}: 12 \mathrm{~h}$ light:dark cycle year round. Twice weekly, animals were fed a diet of carrots, raisins, and iceberg lettuce. Intact animals were maintained under these conditions as an intermolt comparison to multiple leg autotomy (MLA).

\section{Multiple Leg Autotomy}

Multiple leg autotomy of all eight walking legs was performed for thirty-six crabs collected during May 2013. After MLA, animals were transferred to individual plastic cages lined with sand moistened with 10 p.p.t. Instant Ocean. Cages were then shielded from room lighting using a cloth to shorten the premolt period (Bliss and Boyer, 1964). The size of the growing limb bud of the third walking leg on the right side was measured regularly. Progression through the molt stages was tracked using an R-index, calculated based on the ratio of the size ( $\mathrm{mm}$ ) of the limb bud to the width (mm) of the carapace (Covi et al., 2010; Yu et al., 2002). The R-index was used as an indicator of molt stage, which was later confirmed through quantifying hemolymph ecdysteroid titers at the time of sacrifice. R-indices used to distinguish molt stages were as follows: intermolt $R=8-12$; early premolt $R=12.1-15.9$, mid premolt $R=16-18.9$, late premolt $R=19-22$ (Covi et al., 2010). Twelve animals were sacrificed for each of the early, mid, and late premolt stages. 


\section{Dissection}

All MLA comparison animals were from the group collected in May 2014 and were dissected between October and December 2014. Hemolymph was taken from each animal immediately prior to dissection for quantification of ecdysteroid titers. One hundred microliters of hemolymph were diluted in three hundred microliters of ethanol for each animal. Samples were stored at $-20^{\circ} \mathrm{C}$ until overnight shipment to the Bodega Marine Laboratory where an ELISA assay was performed by Dr. Ernest Chang to determine ecdysteroid content.

Anesthetization of animals was accomplished by immersion in crushed ice for five to ten minutes, followed immediately by dissection of each YO pair. YOs were rinsed in crab saline and flash frozen in liquid nitrogen to prevent degradation. Samples were stored at $-80^{\circ} \mathrm{C}$ until overnight shipment on dry ice to California Polytechnic State University, San Luis Obispo. Once received, samples were again stored at $-80^{\circ} \mathrm{C}$.

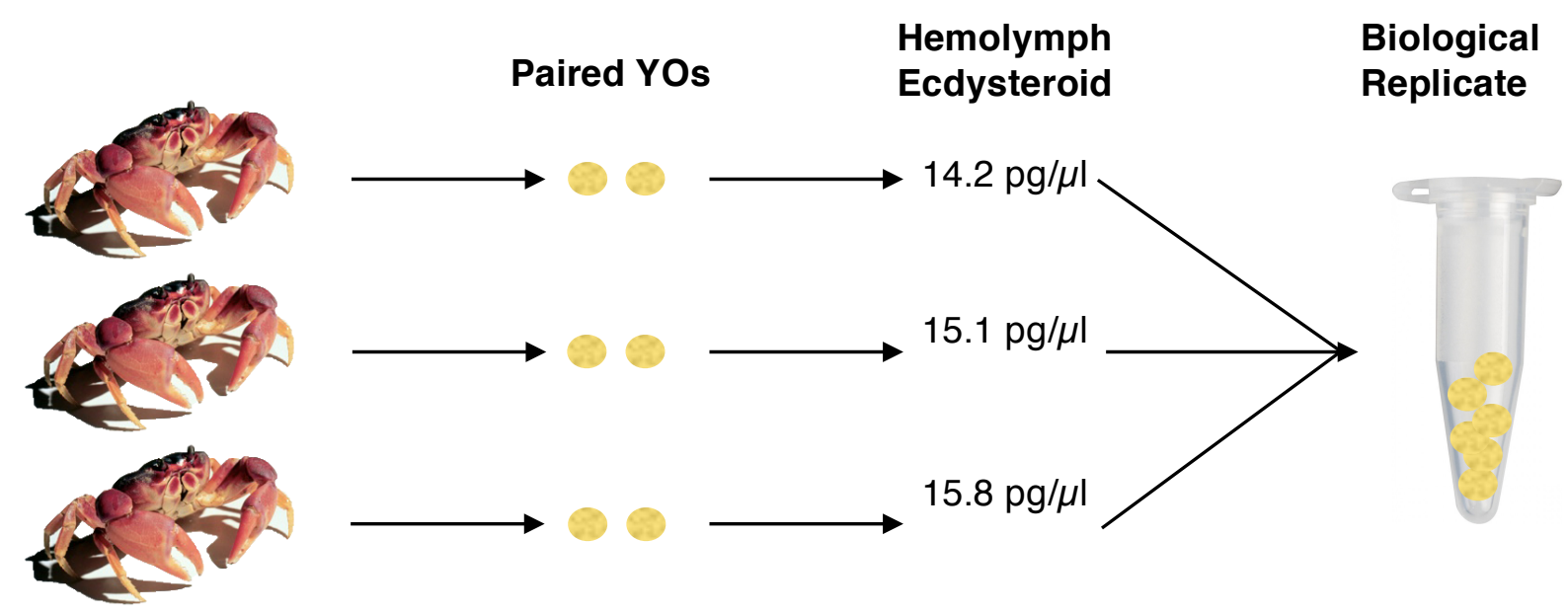

Figure 11. Composition of biological replicates used for analysis of each molting stage. YO pairs from each animal were pooled with animals of similar hemolymph ecdysteroid titers for early, mid, and late premolt stages to produce 4 biological replicates for each molt stage. 


\section{Homogenization and Protein Isolation}

Preliminary data from Carcinus maenas revealed that a single pair of YOs does not produce sufficient protein concentrations for analysis (Hamer, 2015). Due to their small size, three pairs of YOs were pooled from three animals with similar hemolymph ecdysteroid titers to make biological replicates for each treatment group, after first being sorted by R-index for early, mid, and late premolt stages (Fig. 11). Biological replicates for intermolt animals were pooled randomly and Rindices were not measured, as intermolt animals are not actively regenerating limb buds. Four biological replicates were used for each stage for MLA comparisons: intermolt (intact), early, mid, and late premolt (Table 2). Due to an anomaly, one crab with a large R-index (23.9) but a low hemolymph ecdysteroid titer $(68.7 \mathrm{pg} / \mu \mathrm{l})$ was omitted from analysis and the late premolt replicate 4 consisted of only two animals.

Individual samples were pooled into biological replicates immediately following removal from $-80^{\circ} \mathrm{C}$. Biological replicates were then homogenized using a glass homogenizer on ice in 0.6 $\mathrm{mL}$ homogenization buffer (7 $\mathrm{M}$ urea, $2 \mathrm{M}$ thiourea, 1\% ASB-14, $40 \mathrm{mM}$ Tris-base, 0.001\% bromophenol blue, $40 \mathrm{mM}$ dithiothreitol (DTT), and 0.5\% immobilize $\mathrm{pH} \mathrm{3-10} \mathrm{gradient} \mathrm{(IPG)}$ buffer). Samples were then transferred to $2 \mathrm{~mL}$ siliconized microcentrifuge tubes and kept at room temperature for one hour to aid in protein solubilization. Following a 30 minute centrifugation at $16,000 \mathrm{~g}$, the supernatant was aliquoted into two fresh $2 \mathrm{~mL}$ siliconized microcentrifuge tubes per sample, each containing approximately $0.3 \mathrm{~mL}$ of solubilized protein solution. The gelatinous pellets containing insoluble cellular components were discarded. To each aliquot, $1.6 \mathrm{~mL}$ of icecold 10\% trichloroacetic acid (TCA) in acetone was added. All samples were then vortexed briefly and stored at $-20^{\circ} \mathrm{C}$ overnight to allow for precipitation of proteins from the solution. 
Table 2. Organization of YO samples into biological replicates of groups of three YO-pairs. Samples were pooled according to molt stage first according to R-index, followed by similar hemolymph ecdysteroid values.

\begin{tabular}{cccccc}
\multicolumn{2}{c}{$\begin{array}{l}\text { Intermolt } \\
\text { Biological }\end{array}$} & $\begin{array}{c}\text { Hemolymph } \\
\text { Replicate }\end{array}$ & $\begin{array}{c}\text { Animal \# } \begin{array}{c}\text { Ecclysteroid } \\
\text { (pg//) }\end{array} \\
\text { R-index }\end{array}$ & Comments & $\begin{array}{c}\text { Delta 2D } \\
\text { Labeling }\end{array}$ \\
\hline 1 & 501 & 3.59 & - & Intact & Intermolt (07) \\
1 & 502 & 5.11 & - & Intact & Intermolt (07) \\
1 & 500 & 6.58 & - & Intact & Intermolt (07) \\
2 & 504 & 8.08 & - & Intact & Intermolt (08) \\
2 & 505 & 6.56 & - & Intact & Intermolt (08) \\
2 & 506 & 5.08 & - & Intact & Intermolt (08) \\
3 & 507 & 4.57 & - & Intact & Intermolt (09) \\
3 & 508 & 7.54 & - & Intact & Intermolt (09) \\
3 & 509 & 4.38 & - & Intact & Intermolt (09) \\
4 & 510 & 4.84 & - & Intact & Intermolt (10) \\
4 & 511 & 4.57 & - & Intact & Intermolt (10) \\
4 & 512 & 4.57 & - & Intact & Intermolt (10)
\end{tabular}

\section{Early Premolt}

\begin{tabular}{|c|c|c|c|c|c|}
\hline $\begin{array}{l}\text { Biological } \\
\text { Replicate }\end{array}$ & Animal\# & $\begin{array}{c}\text { Hemolymph } \\
\text { Ecolysteroid } \\
(p g / \mu l)\end{array}$ & R-index & Comments & Delta 2D Labeling \\
\hline 1 & $P E B$ & 14.2 & 12.2 & MLA & Early Premolt (01) \\
\hline 1 & $\mathrm{FE}$ & 15.1 & 12.2 & MA & Early Premolt (01) \\
\hline 1 & PE11 & 15.8 & 12.2 & MA & Early Premolt (01) \\
\hline 2 & FE4 & 16.5 & 13.6 & MA & Early Premolt (02) \\
\hline 2 & 五 & 172 & 12.5 & MA & Early Premolt (02) \\
\hline 2 & PE9 & 19.4 & 13.0 & MA & Early Premolt (02) \\
\hline 3 & PE10 & 21.7 & 12.9 & MA & Early Premolt (03) \\
\hline 3 & PE3 & 30.0 & 13.1 & MA & Early Premolt (03) \\
\hline 3 & 円 & 35.7 & 13.8 & MA & Early Premolt (03) \\
\hline 4 & PE2 & 58.7 & 13.5 & MA & Early Premolt (04) \\
\hline 4 & PE1 & 104.1 & 13.2 & MA & Early Premolt (04) \\
\hline 4 & PE12 & 124.9 & 13.9 & MA & Early Premolt (04) \\
\hline
\end{tabular}

Mid Premolt

Late Premolt

\begin{tabular}{cccccc}
$\begin{array}{c}\text { Biological } \\
\text { Replicate }\end{array}$ & $\begin{array}{c}\text { Hemolymph } \\
\text { Animal \# } \begin{array}{c}\text { Ectysteroid } \\
\text { (pg//l) }\end{array}\end{array}$ & R-index & Comments & $\begin{array}{c}\text { Delta2D } \\
\text { Labeling }\end{array}$ \\
\hline 1 & PMT & 41 & 17.4 & MLA & MidPremolt (05) \\
1 & PM6 & 41.9 & 16.8 & MA & Mid Premolt (05) \\
1 & PM9 & 45 & 16.4 & MA & Mid Premolt (05) \\
2 & PM4 & 49.6 & 16.2 & MA & Mid Premolt (06) \\
2 & PM5 & 52.2 & 16.7 & MA & Mid Premolt (06) \\
2 & PM7 & 68.3 & 16.5 & MA & Mid Premolt (06) \\
3 & PM12 & 121.2 & 16.1 & MA & Mid Premolt (07) \\
3 & PMB & 118.5 & 16.8 & MA & Mid Premolt (07) \\
3 & PMB & 109.3 & 16.9 & MA & Mid Premolt (07) \\
4 & PMR & 131.4 & 16.0 & MA & Mid Premolt (08) \\
4 & PM10 & 119.2 & 17.5 & MA & Mid Premolt (08) \\
4 & PM11 & 188.3 & 18.1 & MA & Mid Premolt (08)
\end{tabular}

\begin{tabular}{|c|c|c|c|c|c|}
\hline $\begin{array}{l}\text { Biological } \\
\text { Replicate }\end{array}$ & Animal\# & $\begin{array}{l}\text { Hemolymph } \\
\text { Ecclysteroid } \\
\text { (pg/ } / \mathrm{l})\end{array}$ & R-index & Comments & Delta 2D Labeling \\
\hline 1 & PQ & 20.7 & 219 & MLA & Late Premolt (09) \\
\hline 1 & $\mathrm{P7}$ & 229.4 & 20.5 & MLA & Late Premolt (09) \\
\hline 1 & P8 & 238.7 & 21.0 & MA & Late Premolt (09) \\
\hline 2 & P5 & 242.7 & 21.1 & MA & Late Premolt (10) \\
\hline 2 & $\mathrm{R3}$ & 252.1 & 24.0 & MA & Late Premolt (10) \\
\hline 2 & PL10 & 253.9 & 22.2 & MA & Late Premolt (10) \\
\hline 3 & PL11 & 314.5 & 19.7 & MA & Late Premolt (11) \\
\hline 3 & $\mathrm{R} 24$ & 318.7 & २3.6 & MA & Late Premolt (11) \\
\hline 3 & R6 & 370.7 & 21.3 & MA & Late Premolt (11) \\
\hline 4 & R2 & 390.4 & 24.6 & MA & Late Premolt (12) \\
\hline 4 & Pl1 & 515.7 & 21.1 & MA & Late Premolt (12) \\
\hline
\end{tabular}

Following overnight incubation, samples were centrifuged at $18,000 \mathrm{xg}$ for 15 minutes at $4^{\circ} \mathrm{C}$. Supernatants were discarded as waste and $1.6 \mathrm{~mL}$ cold $100 \%$ acetone was added to rinse the pellets and vortexed. Samples were then centrifuged again at $18,000 \mathrm{xg}$ for 15 minutes at $4^{\circ} \mathrm{C}$. Following the rinse, the remaining supernatant was decanted and pellets were air dried briefly. To each pellet, $0.4 \mathrm{~mL}$ of rehydration buffer (7 $\mathrm{M}$ urea, $2 \mathrm{M}$ thiourea, 2\% 3-[(3Cholamidopropyl)dimethylammonio]-1-propanesulfonate (CHAPS), $2 \%$ octylphenoxypolyethoxyethanol (Nonidet- P-40), 0.002\% bromophenol blue, 0.5\% pH 3-10 IPG buffer, and $100 \mathrm{mM}$ dithioerythritol (DTE)) was added and samples were vortexed briefly, then 
stored at $4^{\circ} \mathrm{C}$ overnight to rehydrate the precipitated proteins. Samples were then vortexed briefly and centrifuged at $16,000 \mathrm{~g}$ for ten minutes at room temperature. Aliquots were then pooled once again into biological replicates by decanting the respective supernatants into a fresh $2 \mathrm{~mL}$ siliconized microcentrifuge tube; the gelatinous pellets were discarded. From each sample, a 4.5 $\mu 1$ aliquot was taken in a new siliconized tube before all samples were stored at $-80^{\circ} \mathrm{C}$. The $4.5 \mu 1$ aliquots were used to determine protein concentration for each sample following the protocol for the GE Healthcare 2D Quant Kit (Chicago, IL, USA).

\section{Two-Dimensional Gel Electrophoresis}

Isoelectric focusing across an immobilized $\mathrm{pH}$ 5-8 ReadyStrip ${ }^{\mathrm{TM}} 11 \mathrm{~cm}$ long IPG strip (Bio-Rad Laboratories, Hercules, CA, USA) was used to load $400 \mu \mathrm{g}$ of isolated protein from each sample (Berth et al., 2007; Magdeldin et al., 2014). Prior to initiating the protocol, $1.6 \mathrm{~mL}$ of mineral oil was added to each lane in the isoelectric focusing chamber to prevent overheating. Isoelectric focusing took place through a six step protocol over a 24-hour period. Initially, proteins were passively rehydrated for 5 hours at $0 \mathrm{~V}$, then actively rehydrated for 12 hours at $50 \mathrm{~V}$. The next three steps consisted of an increase of $500 \mathrm{~V}$ over one hour, $1000 \mathrm{~V}$ over one hour, and 8000 $\mathrm{V}$ over 2.5 hours, followed by a terminal holding step at $500 \mathrm{~V}$. Strips were then blotted clean of mineral oil and stored at $-80^{\circ} \mathrm{C}$ overnight.

In preparation for SDS-PAGE, IPG strips were removed from the freezer and incubated in $1.6 \mathrm{~mL}$ rehydration buffer $(375 \mathrm{mM}$ Tris-Base, $6 \mathrm{M}$ urea, $30 \%$ glycerol, $2 \% \mathrm{SDS}, 0.002 \%$ bromophenol blue) with $65 \mathrm{mM}$ DTT for 15 minutes at room temperature to disrupt any disulfide bonds. Remaining rehydration buffer was then decanted and replaced with $1.6 \mathrm{~mL}$ equilibration buffer (375 mM Tris-Base, $6 \mathrm{M}$ urea, 30\% glycerol, 2\% SDS, 0.002\% bromophenol blue) and 135 $\mathrm{mM}$ iodoacetamide to quench any remaining DTT. Strips were once again incubated for 15 
minutes at room temperature, then suspended in a $0.8 \%$ agarose layer on top of a $12 \%$ polyacrylamide gel. Gels were run for 50 minutes at $200 \mathrm{~V}$ in actively cooled $\left(10^{\circ} \mathrm{C}\right)$ running buffer (25 mM Tris-base, 192 mM glycine, and 0.1\% SDS) using Criterion ${ }^{\mathrm{TM}}$ Dodeca $^{\mathrm{TM}}$ Cells. Gels were then stained overnight in Coomassie Blue G-250 staining solution, followed by periodic rinsing with Milli-Q water to destain gels until background Coomassie stain was no longer visible. An Epson 1280 transparency scanner was used to create digital images of each gel.

\section{Gel image analysis with Delta $2 D$}

Gel images were analyzed using Decodon Delta 2D (Version 4.3). Match vectors between protein spots were automatically created using the program's SmartVectors ${ }^{\mathrm{TM}}$ function. Due to some faint spots and computer error, all vectors were then then manually checked and corrected and new vectors were added as needed. All gel images were then automatically overlaid to create a fused gel image representative of the average relative spot intensity for each protein spot detected on the gels (the so-called proteome map). Each protein spot with distinct borders and a visual intensity greater than that of the background was assigned a number and a spot boundary, and transferred to each gel, enabling consistency across all gel images.

\section{Mass Spectrometry}

Protein spots were then manually excised from the gels using a tissue punch (Beecher Instruments; Prairie, WI, USA) and stored in individual $0.6 \mathrm{~mL}$ safe-lock microcentrifuge tubes (Eppendorf; Hamburg, Germany) at $-20^{\circ} \mathrm{C}$ until trypsin digestion. Spots that were faintly stained were pooled from two gels in order to increase the likelihood of protein identification. Upon removal from $-20^{\circ} \mathrm{C}, 200 \mu \mathrm{l}$ of destaining buffer $\left(25 \mathrm{mM} \mathrm{NH}_{4} \mathrm{HCO}_{3} / 50 \%\right.$ acetonitrile $)$ was added to each tube, and tubes were placed on a shaker for 45 minutes at room temperature. Used destaining buffer was then removed from each tube and discarded, being careful to leave the gel 
plug in each tube, and replaced by $200 \mu 1$ of $100 \%$ acetonitrile. Tubes were incubated at room temperature for $10 \mathrm{~min}$. After incubation, acetonitrile was removed and the now small, white gel plugs were allowed to dry by leaving tubes with the lids open under a fume hood for 5 minutes. Following drying, $15 \mu 1$ of trypsin solution $\left(40 \mathrm{mM} \mathrm{NH}_{4} \mathrm{HCO}_{3} / 10 \%\right.$ acetonitrile with $165 \mu \mathrm{g} / 15 \mu 1$ trypsin) was added to each tube, and all tubes were incubated at $37^{\circ} \mathrm{C}$ overnight.

The following morning, $50 \mu 1$ of analyte solution ( $0.1 \%$ trifluoroacetic acid (TFA) $/ 100 \%$ acetonitrile [2:1]) was added to each sample and incubated at room temperature for 60 minutes. The remaining liquid was then removed from each tube and placed in a fresh, labeled $0.6 \mathrm{~mL}$ SafeLock microcentrifuge tube. Samples were then concentrated in a Speed-Vac at $45^{\circ} \mathrm{C}$ until dry (about 50 minutes). Directly to the dried samples $3 \mu 1$ of TA-30 solvent (acetonitrile/0.1\% TFA [30:70]) was pipetted to each tube to dissolve the dried sample. On an MTP AnchorChip 384 BC (Bruker Daltonics, Inc.; Billerica, MA, USA) plate, $1 \mu \mathrm{l}$ of each sample was plated to two separate anchors of the same column but different row (i.e. A1 and B1). Samples were allowed to dry under the fume hood for $10-15 \mathrm{~min}$ before $1 \mu \mathrm{l}$ of matrix solution $(0.7 \mathrm{mg} / \mathrm{ml} \mathrm{HCCA}$ in $85 \%$ acetronitrile, $15 \% \mathrm{H}_{2} \mathrm{O}, 0.1 \%$ TFA, $\left.1 \mathrm{mM}\left(\mathrm{NH}_{4}\right) \mathrm{H}_{2} \mathrm{PO}_{4}\right)$ was placed onto each spot and allowed to dry for an additional $15 \mathrm{~min}$. Finally, the first of each pair of rows was washed by lightly pipetting $5 \mu \mathrm{l}$ of cold HPLC water up and down.

A matrix-assisted laser desorption/ionization tandem time-of-flight [(MALDI-ToF/ToF) UltraFlex II; Bruker Daltonics, Inc.] mass spectrometer was used to obtain peptide mass fingerprints (PMFs). Internal calibration was performed using porcine trypsin (Promega; Madison, WI, USA). Flex Analysis (version 3.0; Bruker Daltonics, Inc.) was used to detect peptide peaks for both MS and tandem MS (MS/MS). 
Identification of proteins took place using Mascot (Version 2.4; Matrix Science Inc., Boston, MA, USA). PMF and MS/MS results for each protein spot were compared against a Gecarcinus lateralis RNA-seq database consisting of 1,389,474 sequences (Das et al., 2016). If no matches were found, comparisons continued along a series of databases from a Petrolisthes EST (Porcelain Crab Array Database) consisting of 19,000 sequences, NCBI Arthropoda EST consisting of 30,599,286 sequences, and NCBI Metazoa EST which contains 9,560,026 GenBank, PIR, SWISS-PROT, PRF, and PDB gene entries. Peptide matches with a MOWSE score greater than 46 and at least two peptide matches per protein were used in statistical analysis.

\section{Statistical Analysis}

A 1000 permutation one-way ANOVA $(\alpha=0.05)$ was performed in Delta 2D with intermolt, early premolt, mid premolt, and late premolt treatment groups. Hierarchical clusters (HCL) were created using a Pearson correlation coefficient, clustering proteins by similarities in abundance (spot intensity) among the molt stages. Principal component analyses (PCA) were also performed using the built-in statistical software for Delta 2D, clustering each gel image by similarities in protein expression. 


\section{RESULTS AND DISCUSSION}

\section{Protein Detection, Identification, and Abundance Changes}

A fused gel image was created in Delta 2D in order to visualize individual protein spots, or isoforms of individual proteins (Fig. 12). Of the 457 protein spots detected on the fused gel image, 191 (41.8\%) were positively identified using MALDI-ToF/ToF mass spectrometry (Table 5). Hierarchical clusters created using Pearson correlation coefficient values were generated for all identified proteins in order to visualize patterns in changes of protein abundance among the molt stages. Of the 230 total proteins that changed significantly in abundance among the molt stages (one-way ANOVA p $\leq 0.05$ ), 109 (47.4\%) were positively identified (Fig. 27A-C).

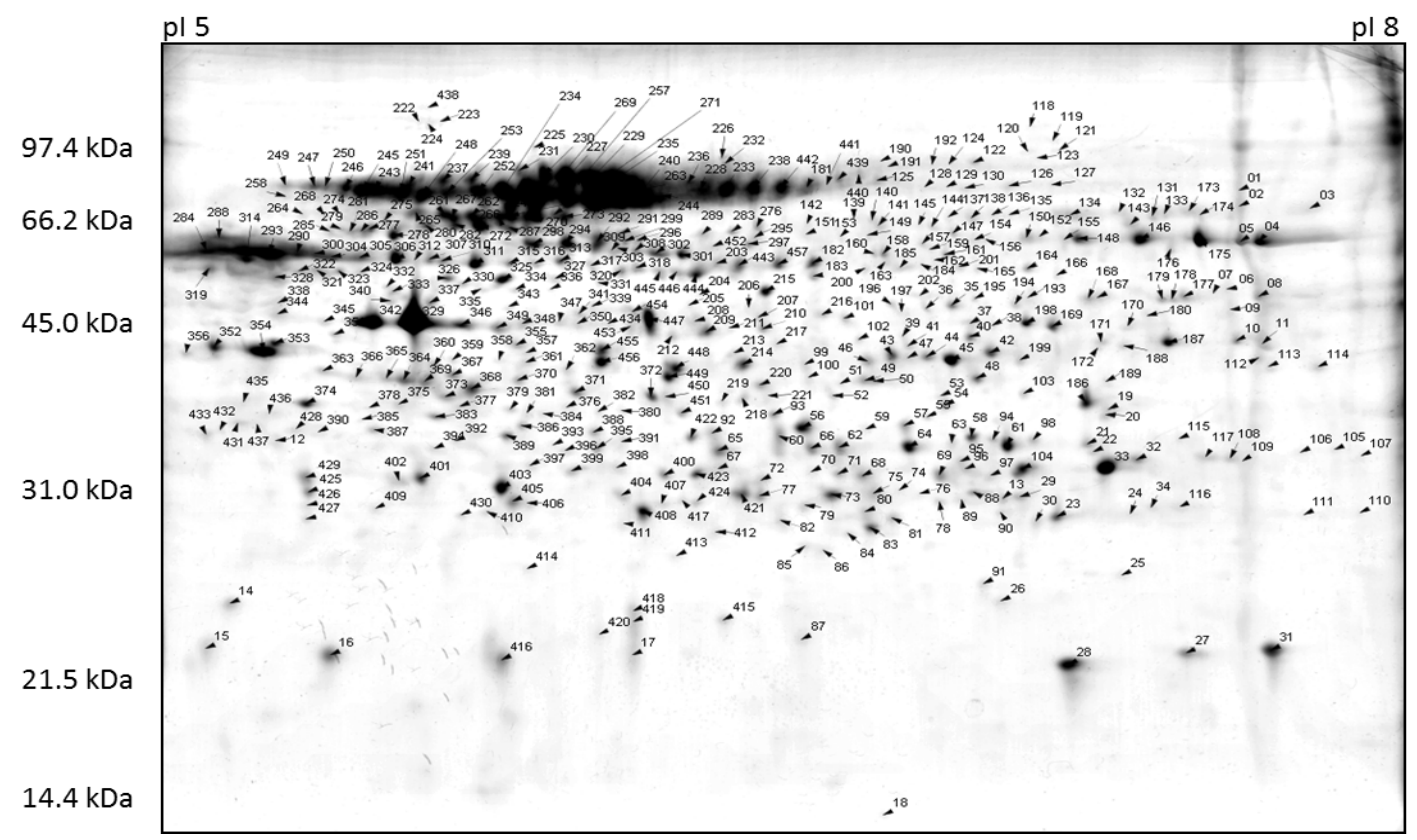

Figure 12. Two-dimensional gel proteome map composed of gels from all molt stages. Spot numbers represent all 457 protein spots detected from the G. lateralis $\mathrm{YO}$ in intermolt, early, mid, and late premolt stages. The average pixel density from this fused image was normalized to determine changes in pixel density (protein abundance) among each gel image in each molt stage. All protein spots were manually excised from gels for identification using MALDI-ToF/Tof mass spectrometry, of which 191 were positively identified. 
Individual HCLs were created for functional groups, which contribute significantly to changes in protein abundance among the molt stages.

\section{Principal Component Analysis}

Principal component analysis was used to visualize the patterns in overall protein expression among molt stages for all identified proteins (Fig. 13A), as well as all proteins which changed significantly in abundance, regardless of identification (Fig. 13B). Individual points represent a single gel image. Points that are tightly clustered together have similar patterns of protein expression, while points that are separated among either the $\mathrm{x}$-axis $(\mathrm{PC} 1)$ or the $\mathrm{y}$-axis (PC2) have different patterns of protein expression.

A distinct contrast in protein expression from the intermolt stage to the premolt stages is easily visualized through principal component analyses. Observation of all detected proteins displays a separation of the intermolt stage from all three premolt stages along the first axis (PC1) which explains nearly $30 \%$ of the variation in protein abundances among the molt stages (Fig. 13A). Further, observation of only those proteins which changed significantly in abundance among the molt stages (one-way ANOVA, $\mathrm{p} \leq 0.05$ ) revealed a separation of intermolt from all three premolt stages, which explains nearly $46 \%$ of the variation in protein abundances (Fig. 13B). Taken together, these analyses indicate a distinct 'on-off' switch of the YO from the basal intermolt stage to the active premolt stages. Those proteins contributing to such a separation of intermolt from premolt were identified through observation of eigenvalues. The most important proteins contributing to the separation of intermolt from premolt are predominantly involved in energy metabolism, oxidative stress, cytoskeleton, and hemolymph (Tables 3 and 4) and will be discussed in further detail for each functional group. 
The second principal component (PC2), which explains $11 \%$ of the variation of all detected proteins (Fig. 13A) and 12\% of the variation of significant proteins (Fig. 13B), is characterized by the separation of the premolt stages. Notably, the mid premolt stage is distinct from both early and late premolt in both analyses. Physiology of the mid premolt YO is of particular interest due to the YO entering the "committed" stage, where it is refractory to inhibition by MIH or limb bud autotomy (Chang and Mykles, 2011). The mid premolt YO is characterized by an increase in ecdysteroid synthesis, reaching peak hemolymph ecdysteroid titers just before transition to the late premolt YO. Total protein expression reveals separate groupings of the early premolt and late premolt YOs along PC1 and PC2 (Fig. 13A), however, analysis of only those proteins which changed significantly in abundance reveals a high degree of overlap in protein expression (Fig. 13B).

Here it is important to note that those proteins which contribute most to the separation along the second principal component axis for all expressed proteins (Table 3) are dissimilar to those contributing to the separation along PC2 for only significantly changing proteins (Table 4). This is in contrast to those proteins contributing to the separation along PC1 for all expressed proteins and all significant proteins, which are remarkably similar. This not only supports the physiological 'on-off' switch hypothesis, but also highlights the importance of all expressed proteins in the YO, regardless of statistical significance. As such, the following discussion will focus on those proteins that change significantly in abundance with molt stage, particularly those which contribute to separation along PC1 or PC2, but will also include proteins which do not change significantly in abundance but nonetheless are important for physiological function of the YO at any given stage. 


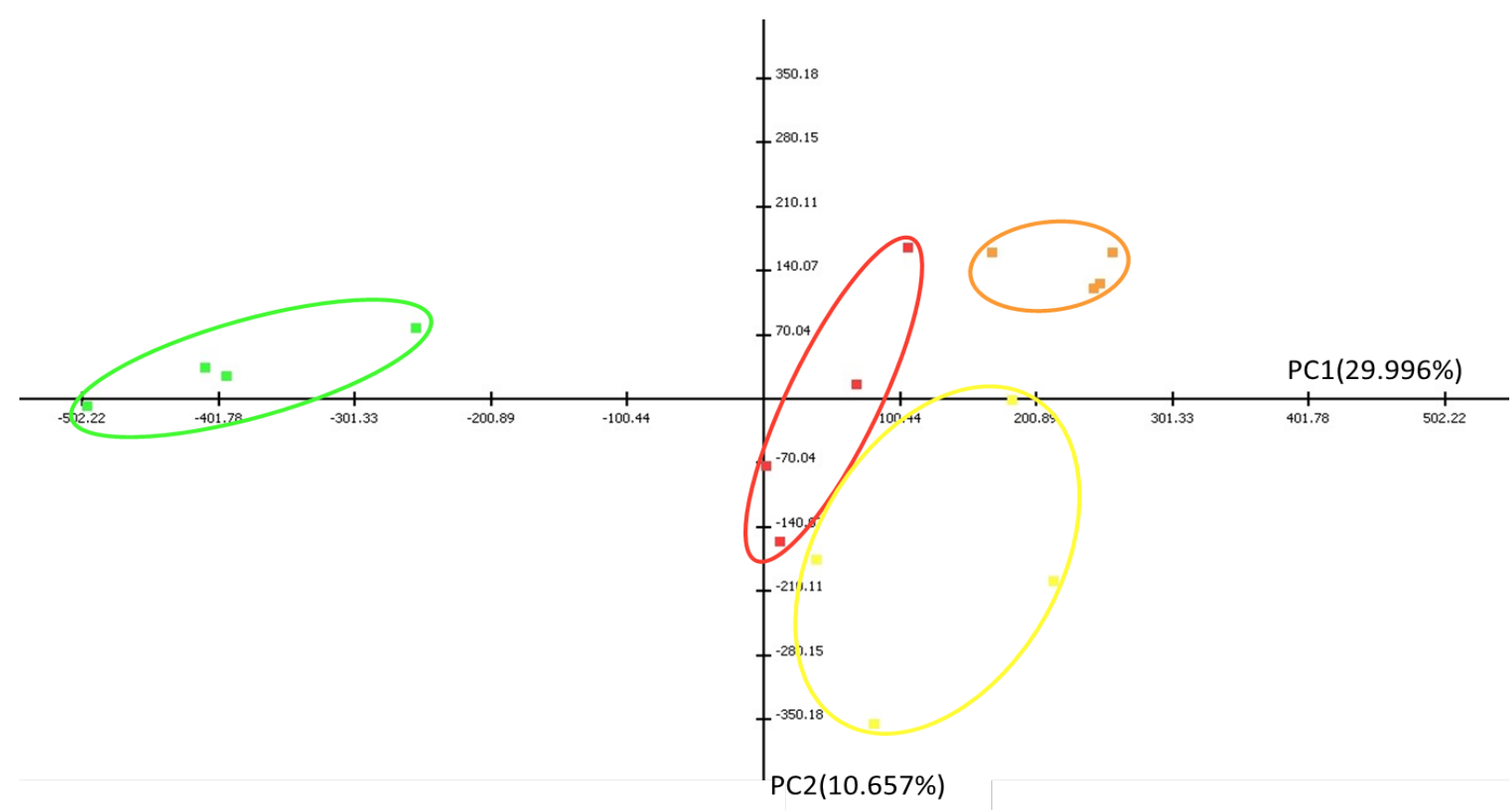

Figure 13A. Principal component analysis displaying all proteins within the Y-organ of Gecarcinus lateralis over the course of the molt cycle induced by MLA. Individual points represent a single composite gel image composed of one biological replicate (3 pairs of Y-organs from animals in the same molting stage). Points are color coordinated according to stage within the molt cycle, and clustered based on similarities in protein abundance among each gel image. Together, PC1 and PC2 explain $40.653 \%$ of the variation in protein abundance observed among molt stages. Individually, PC1 separates the intermolt stage from the premolt stages and explains $29.996 \%$ of the variation in protein abundance observed. PC2, which separates the early, mid, and late premolt stages, explains $10.657 \%$ of the variation in protein abundance seen in the Y-organ.

Table 3. Positive and negative component loading values for all proteins contributing towards principal components 1 and 2.

\begin{tabular}{|c|c|c|c|c|}
\hline \multirow{2}{*}{$\begin{array}{l}\text { Component } \\
\text { Loading Rank }\end{array}$} & \multicolumn{2}{|l|}{ Principal Component 1} & \multicolumn{2}{|l|}{ Principal Component 2} \\
\hline & Protein (spot ID) & Loading Value & Protein (spot ID) & Loading Value \\
\hline \multicolumn{5}{|c|}{ Positive Loadings } \\
\hline 1 & Catalase (148) & 1.60084 & Transglutaminase (288) & 2.00903 \\
\hline 2 & Glyceraldehyde 3-Phosphate Dehydrogenase (186) & 1.56833 & Cathepsin D (352) & 1.91515 \\
\hline 3 & Transglutaminase (237) & 1.56549 & Hemocyanin Subunit 6 (234) & 1.87069 \\
\hline 4 & Protaglandin Reductase 1 (42) & 1.50387 & Heat Shock Protein $70(89)$ & 1.82494 \\
\hline 5 & Malate Dehydrogenase (19) & 1.4797 & Beta-Tubulin (284) & 1.68996 \\
\hline 6 & Phosphopyruvate Hydratase (215) & 1.47657 & C-1-tetrahydrofolate Synthase, Cytoplasmic (182) & 1.65902 \\
\hline 7 & Catalase (146) & 1.44982 & Hemocyanin Subunit $3(272)$ & 1.61892 \\
\hline 8 & Transglutaminase (239) & 1.44418 & Estradiol 17-beta-dehydrogenase 8-like (425) & 1.52353 \\
\hline 9 & Cryptocyanin 2 (235) & 1.43724 & Cryptocyanin 2 (229) & 1.49426 \\
\hline 10 & Transferrin (48) & 1.42885 & Hemocyanin Subunit 6 (262) & 1.34409 \\
\hline \multicolumn{5}{|c|}{ Negative Loadings } \\
\hline 1 & Hemocyanin Subunit 6 (28) & -1.76474 & Protein-Disulfide Isomerase (94) & -2.02992 \\
\hline 2 & Hemocyanin Subunit 6 (27) & -1.75189 & Serine Hydroxymethyltransferase (399) & -1.98976 \\
\hline 3 & Alpha-Tubulin (367) & -1.74998 & Actin (208) & -1.90213 \\
\hline 4 & Hemocyanin Subunit 6 (306) & -1.73912 & Transferrin (67) & -1.89668 \\
\hline 5 & Glyceraldehyde 3-Phosphate Dehydrogenase (56) & -1.73437 & Alpha-Tubulin (290) & -1.86623 \\
\hline 6 & Hemocyanin Subunit 6 (31) & -1.72786 & Beta-Ureidopropionase (209) & -1.73302 \\
\hline 7 & Catalase (178) & -1.72594 & Cathepsin C (16) & -1.69874 \\
\hline 8 & Apoptosis Inducing Factor-3 (337) & -1.70979 & S-formylglutathione Hydrolase (58) & -1.68066 \\
\hline 9 & Beta-Actin (354) & -1.70211 & ES1 Protein Homolog, mitochondrial (81) & -1.64522 \\
\hline 10 & Hemocyanin Subunit 6 (311) & -1.69748 & G Protein Beta $(389)$ & -1.64043 \\
\hline
\end{tabular}




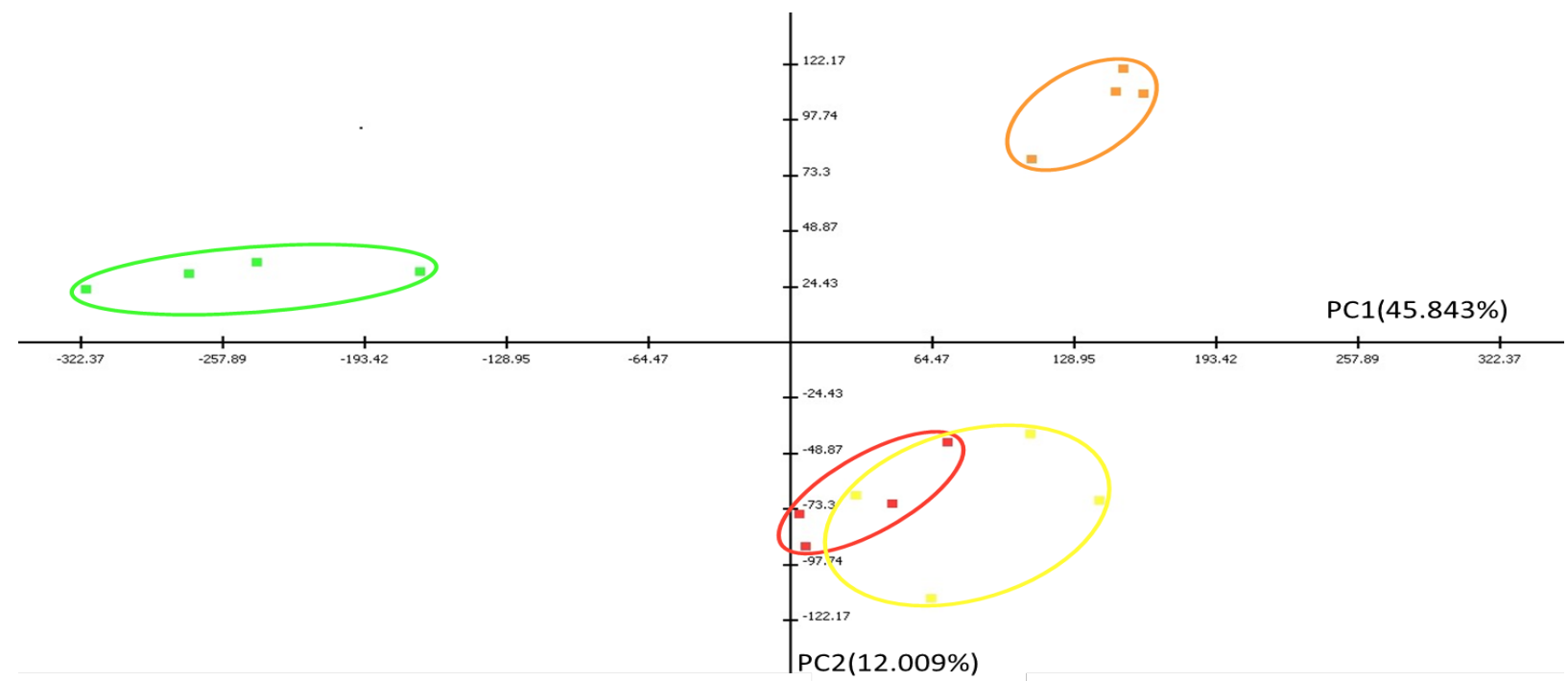

Figure 13B. Principal component analysis displaying all significant proteins within the Y-organ of Gecarcinus lateralis over the course of the molt cycle induced by MLA (One-way permutation ANOVA; $p<0.05)$. This PCA represents $57.852 \%$ of the variation in protein abundance observed among the intermolt, early, mid, and late premolt stages. Individual proteins which contribute to the positive and negative separation among both component 1 and component 2 are indicated in Table 4.

Table 4. Positive and negative component loading values for all significant proteins contributing towards principal components 1 and 2 .

\begin{tabular}{|c|c|c|c|c|}
\hline \multirow{2}{*}{$\begin{array}{l}\text { Component } \\
\text { Loading Rank }\end{array}$} & \multicolumn{2}{|l|}{ Principal Component 1} & \multicolumn{2}{|l|}{ Principal Component 2} \\
\hline & Protein (spot ID) & Loading Value & Protein (spot ID) & Loading Value \\
\hline \multicolumn{5}{|c|}{ Positive Loadings } \\
\hline 1 & Catalase (148) & 1.29263 & Estradiol 17-beta-dehydrogenase 8 (425) & 2.08441 \\
\hline 2 & Glyceraldehyde 3-Phosphate Dehydrogenase (186) & 1.28167 & Rab GDP Dissociation Inhibitor Alpha (332) & 1.93893 \\
\hline 3 & Transglutaminase (237) & 1.2702 & Cathepsin D (352) & 1.93496 \\
\hline 4 & Protaglandin Reductase 1 (42) & 1.22386 & Catalase (05) & 1.89453 \\
\hline 5 & Phosphopyruvate Hydratase (215) & 1.20734 & F1F0 ATP Synthase Beta Subunit (319) & 1.82366 \\
\hline 7 & Transglutaminase (239) & 1.18338 & Cryptocyanin 2 (229) & 1.49863 \\
\hline 8 & Catalase (146) & 1.17316 & Cryptocyanin 2 (235) & 1.4555 \\
\hline 9 & Transferrin (48) & 1.15621 & Beta-I Tubulin (314) & 1.31749 \\
\hline 10 & Cryptocyanin 2 (235) & 1.1549 & Cryptocyanin (439) & 1.21064 \\
\hline \multicolumn{5}{|c|}{ Negative Loadings } \\
\hline 1 & Hemocyanin subunit $6(28)$ & -1.43524 & Alpha-Tubulin (290) & -1.94946 \\
\hline 2 & Alpha-Tubulin (367) & -1.42677 & Beta-Ureidopropionase-like (209) & -1.75166 \\
\hline 5 & Glyceraldehyde 3-Phosphate Dehydrogenase (56) & -1.40514 & Beta-Tubulin (379) & -1.43173 \\
\hline 6 & Catalase (178) & -1.40397 & G-Protein Beta (389) & -1.35805 \\
\hline 7 & Hemocyanin Subunit 6 (31) & -1.40215 & Beta-Tubulin (304) & -1.34413 \\
\hline 8 & Apoptosis Inducing Factor-3 (337) & -1.38881 & Beta-Tubulin (371) & -1.29064 \\
\hline 9 & Hemocyanin Subunit 6 (311) & -1.38813 & Actin (208) & -1.18245 \\
\hline 10 & Hemocyanin Subunit 6 (302) & -1.37879 & Inorganic Pyrophosphatase (378) & -1.18019 \\
\hline
\end{tabular}




\section{Hemolymph Proteins}

\section{Hemocyanin}

Hemocyanins are copper-containing proteins central to respiration in mollusks and arthropods. Biosynthesis of hemocyanins occurs in the hepatopancreas of brachyurans and subunits of about $75 \mathrm{kDa}$ are assembled into hexameters or multihexamers before release into the hemolymph (Durstewitz and Terwilliger, 1997a). A developmental change in subunit composition of hemocyanins has been shown to occur after the sixth juvenile instar, during which time expression of the 'adult form', subunit 6 , begins and a $50 \%$ increase in oxygen affinity occurs (Durstewitz and Terwilliger, 1997a and 1997b).

We identified thirty-eight isoforms of hemocyanin, all but two of which were identified as subunit 6 . Three distinct clusters representing patterns of change in abundance were identified (Fig. 14). Cluster I is composed mostly of full-length hemocyanins with molecular masses between 62-81 kDa, and are primarily represented in the premolt stages. Previous metabolic studies have revealed a 50 to $1900 \%$ increase in whole animal oxygen consumption prior to ecdysis (Skinner, 1962), indicating the importance of this respiratory protein during these premolt stages. Cluster II is composed of two full-length sequences and two high molecular weight fragments defined by a mass between 39-59 $\mathrm{kDa}$. Increased expression of the hemocyanins in Cluster II is primarily observed in the intermolt stage, with moderate to low expression in early and late premolt. Finally, Cluster III is represented almost exclusively by the intermolt YO and is composed of full sequence, high molecular weight, and low molecular weight (23-24 kDa) fragments, all of which were significantly different in abundance than the premolt stages (one-way ANOVA, $\mathrm{p} \leq 0.05$ ). While identification of hemocyanin in the $\mathrm{YO}$ is likely an artifact of the hemolymph that bathes the YO 
in the cephalothorax, the changes in hemolymph protein composition across the molt stages can still yield valuable information about the physiology of molting.

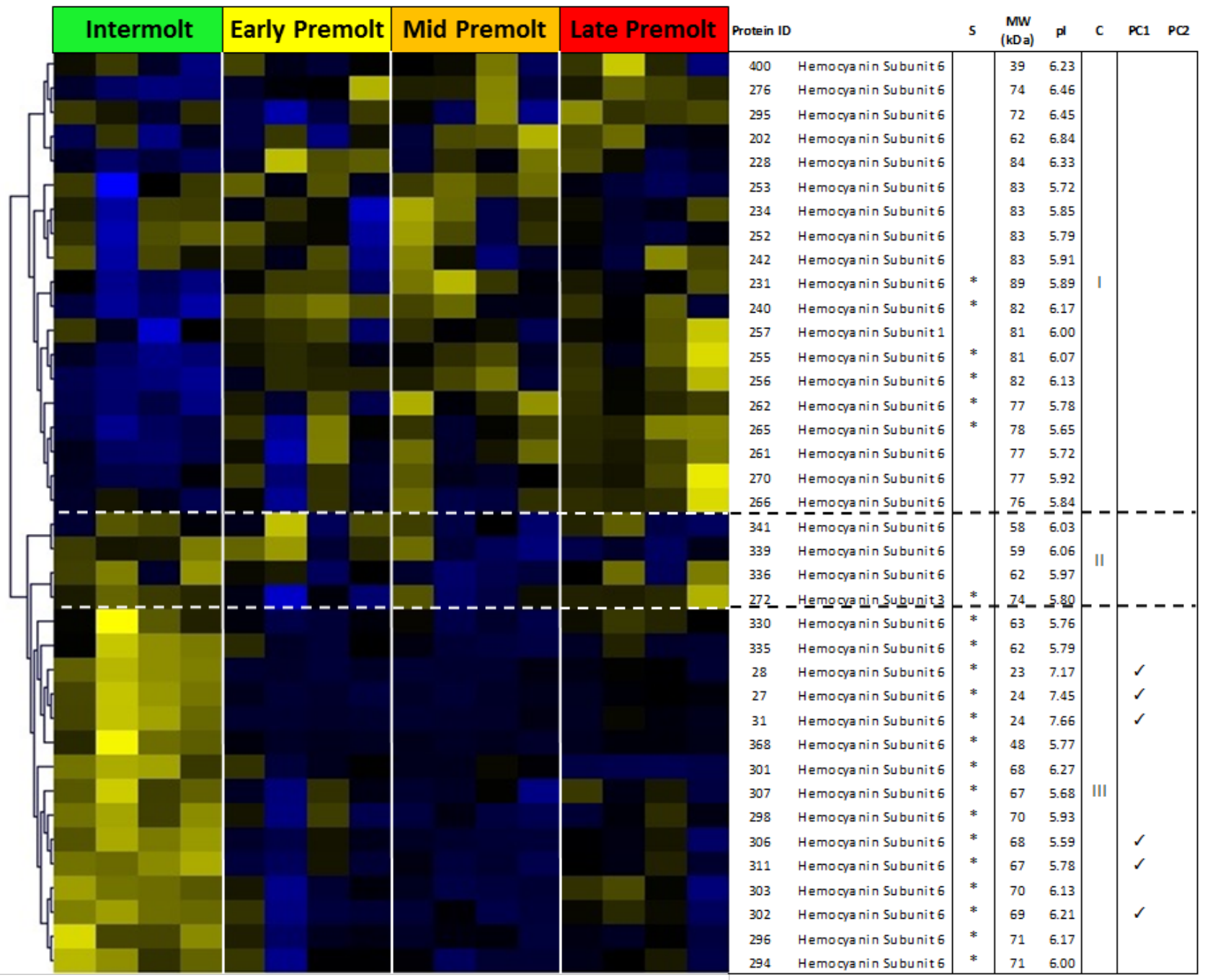

Abundance change:

Figure 14. Hierarchal clustering using Pearson's correlation depicting relative protein abundance (normalized spot volume) for all detected hemocyanin proteins within the Y-organ of Gecarcinus lateralis. Check marks associated with individual protein spots indicate proteins which changed significantly in abundance across the molt stages and exhibit a strong contribution towards the separation among molt stages displayed along the respective component axis (see Fig. 13B and Table 4). 
In addition to their role in respiration, hemocyanins have been shown to play a role as phenoloxidases (POs) upon cleavage by a serine protease (Sritunyalucksana and Söderäll, 2000; Decker and Janice, 2004). Hemocyanin-derived PO activity catalyzes the hydroxylation of monophenols (tyrosine) directly to $o$-diphenols, and the oxidation of $o$-diphenols to $o$-quinones, crucial first steps in both the sclerotization and melanization pathways (Terwilliger and Ryan, 2006, Sritunyalucksana and Söderäll, 2000). Sclerotization, or hardening of the invertebrate cuticle, relies on the cross-linking of proteins and chitin by quinone methide imine amides synthesized by phenoloxidase oxidation of $N$-acetyldopamine (NADA). Melanization, which provides the coloration of the exocuticle as well as both wound-healing and antimicrobial responses, involves the conversion of tyrosine to dopamine (DOPA), and ultimately DOPA to melanin (Sugumaran and Barek, 2016). While both the sclerotization and melanization pathways have overlapping intermediate steps, it is important to note that they can and do occur independently. Melanization in particular can also be activated by the presence of mircobial peptides or damage to the cuticle layer (Sritunyalucksana and Söderäll, 2000).

Cleavage of an N-terminal peptide is thought to activate the PO activity of arthropod hemocyanins, resulting in a high-molecular weight and slightly acidic active phenoloxidase (Decker and Jaenicke, 2004). Presence of slightly acidic, high MW hemocyanin fragments (spots $339,341,368$, and 400), primarily upregulated in intermolt, suggest prophenoloxidase activity. This upregulation is likely associated with both sclerotization and melanization of the cuticle. Down regulation of this prophenoloxidase activity during the premolt stages would aid in synthesis and secretion of the exoskeleton, while preventing complete sclerotization before ecdysis. Interestingly, fumarylacetoacetase (Spot 198), a crucial enzyme in tyrosine metabolism, is increased in abundance, though not significantly, during the premolt stages. Increased 
fumarylacetoacetase activity during the synthesis and secretion of a new, soft exocuticle would aid in preventing premature sclerotization and melanization because tyrosine would be metabolized rather than diverted to the synthesis of melanin (Sugumaran and Barek, 2016). Further evidence for the phenoloxidase-mediated sclerotization and melanization of the crustacean exocuticle has come from a study assessing biosynthesis of hemocyanins over the molt stages which revealed a drastic increase in expression of hemocyanin proteins just after ecdysis (Terwilliger et al., 2005).

Beyond the antimicrobial functions of hemocyanin through activation of the melanization pathway, low molecular weight antifungal and antimicrobial polypeptides have been shown to be homologous to the C-terminal fragment of hemocyanin (Petit et al, 2016; Destoumieux-Garzón et al., 2001; Lee et al., 2003). Three low molecular weight hemocyanin isoforms (spots 27, 28, and 31) were significantly upregulated in the intermolt YO. Investigation of matched peptide sequences from MALDI-ToF/ToF MS revealed that the identified sequences were in fact derived from the C-terminus of the protein. Although the lack of low MW isoforms within the premolt stages is surprising, these data suggest the potential for different immune responses within the YO depending upon molt stage. Later discussion about the clotting protein trangslutaminase, along with other immune response proteins will elucidate on the crustacean immune response.

Cryptocyanin

Cryptocyanin is a crustacean pseudohemocyanin that is similar in sequence, size, and structure to hemocyanin, but lacks three of the six copper-binding histidine residues (Terwilliger et al., 1999). Synchrony between the molt cycle and biosynthesis of cryptocyanin, in which hemolymph titers rise during premolt, peak in the early part of late premolt, drop precipitously before ecdysis, and are virtually undetectable in the hemolymph during intermolt suggests a hormonally regulated mechanism which is independent of that of hemocyanin (Terwilliger et al., 
1999; 2005). In fact, cryptocyanin can reach peak hemolymph titers more than twice as great as hemocyanin during the premolt stages. While eyestalk ablation has been shown to result in a complete cessation of cryptocyanin synthesis, the exact mechanism of control is yet to be elucidated (Terwilliger et al., 2005).

Although lacking in phenoloxidase activity, cryptocyanin has been shown to play a vital role in formation of the exoskeleton. Immunohistochemical studies by Terwilliger et al. (2005) show that cryptocyanin accumulates in 'reserve cells' in the connective tissue, most notably around the epidermis and the extracellular matrix surrounding internal organs, during late premolt. Within hours after ecdysis the pseudohemocyanin is then translocated across the epidermis and incorporated into the new exo- and endocuticle layers. Finally, incorporation into the newly secreted exoskeleton is completed with the cross-linking and proteolytic cleavage of cryptocyanin, another mechanism for sclerotization.

Similar to that mentioned in the discussion of hemocyanin, identification of cryptocyanin in the YO is likely a remnant of hemolymph surrounding the endocrine gland. Hierarchical clustering of the fourteen identified cryptocyanin isoforms displayed two distinct clusters (Fig. 15). The first cluster is composed of just two isoforms, both of which are apparently more abundant in intermolt than the premolt stages. As previously mentioned, little to no cryptocyanin has been detected in the hemolymph of intermolt animals. However, based on the anatomical location of the YO along the endocuticle in the cephalothorax, the identification of these intermolt isoforms could be associated with sclerotization of the endocuticle. This hypothesis is further supported by the low molecular weight of these two isoforms, indicating some form of proteolytic cleavage.

The second cluster is represented by twelve isoforms of cryptocyanin, nine of which change significantly in abundance over the course of the molt cycle. These cryptocyanin isoforms 
show patterns of abundance highest in early and mid premolt, with moderate abundance in late premolt and were virtually undetected in intermolt. Although we detected a peak in cryptocyanin in the YO during mid premolt rather than that described in late premolt by Terwilliger in 2005, this is likely representative of the sequestration of cryptocyanin into reserve cells during late premolt. Here it is also important to reiterate that the observation of such hemolymph proteins within the $\mathrm{YO}$ are likely not entirely representative of the exact composition of the hemolymph at any given stage. Overall, our data strongly corroborates those findings of Terwilliger and her colleagues over the years. More recent evidence has suggested that cryptocyanin may also act as a carrier protein for hormones, divalent cations, or other molecules and may even be part of the immune response during premolt (Terwilliger, 2012). Although still fairly preliminary in
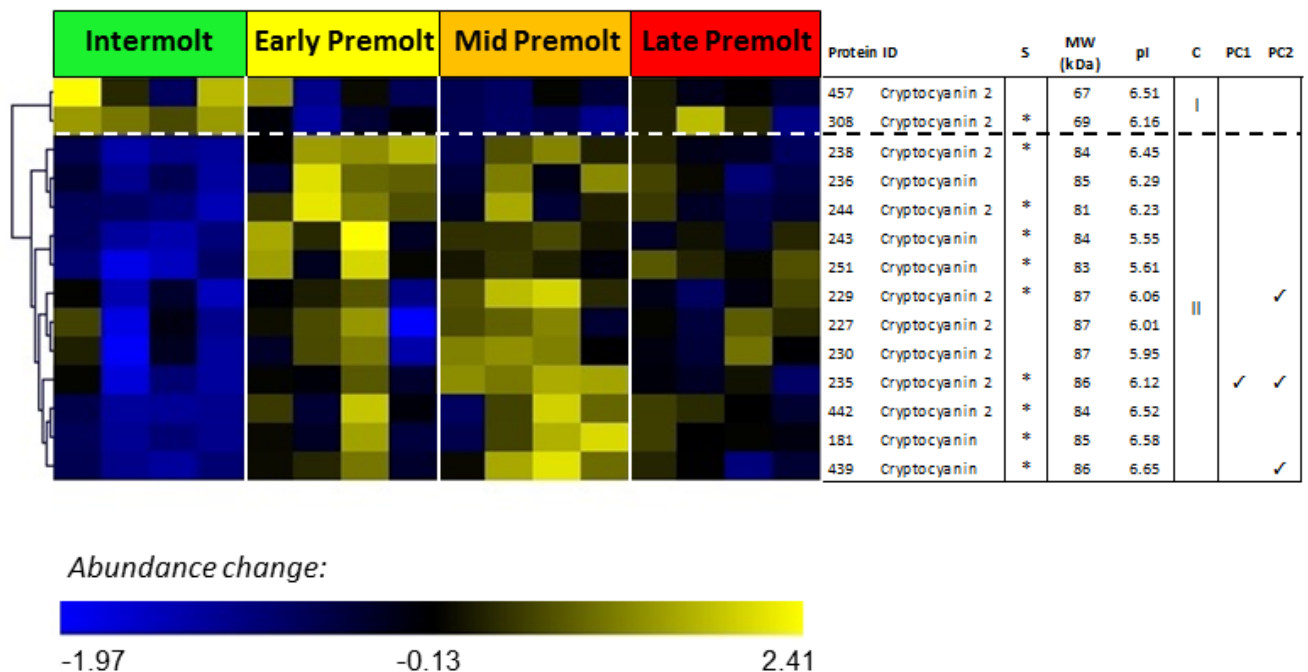

Figure 15. Hierarchal clustering using Pearson's correlation depicting relative protein abundance (normalized spot volume) for all detected cryptocyanin proteins within the Y-organ of Gecarcinus lateralis. Check marks associated with individual protein spots indicate proteins which changed significantly in abundance across the molt stages and exhibit a strong contribution towards the separation among molt stages displayed along the respective component axis (see Fig. 13B and Table 4). 
understanding of these physiological roles, they remain harmonious with our observed changes in cryptocyanin abundance over the molt cycle.

\section{Transglutaminase}

Another protein likely entering the YO through the hemolymph is hemocyte protein glutamine gamma-glutamyltransferase, a member of the transglutaminase (TGase) family. TGases are calcium-dependent enzymes, that catalyze post-translational modifications of proteins, specifically by catalyzing acyl-transfers between peptidal glutamine residues and primary amines (Smethurst and Griffin, 1996). Several TGases have been implicated in the cross-linking of cuticle proteins in horseshoe crabs (Matsuda et al., 2007), as well as plasma clotting in other crustaceans (Lin et al., 2008). Known substrates of TGases include biogenic amines, such as the previously mentioned DOPA, as well as cytoskeletal proteins actin and tubulin, lipoproteins such as the crustacean clotting protein, and the energetically important glyceraldehyde 3-phosphate dehydrogenase (Lai et al., 2017).

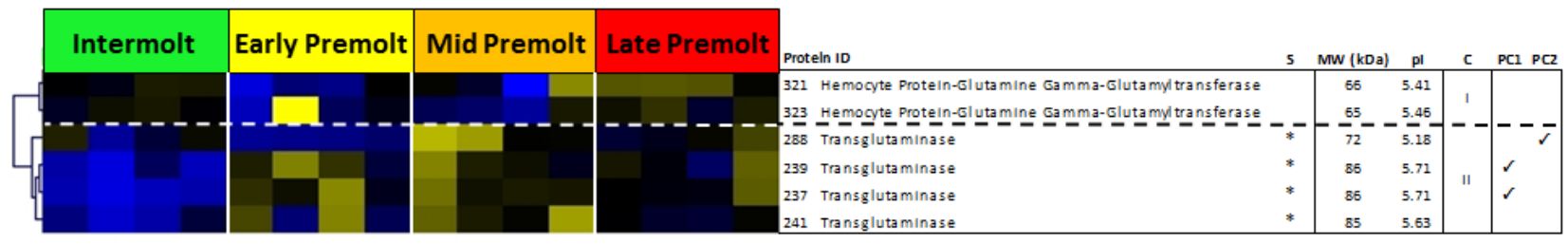

Abundance change:

$-1.96$

0.05

3.03

Figure 16. Hierarchal clustering using Pearson's correlation depicting relative protein abundance (normalized spot volume) for all detected transglutaminase within the Y-organ of Gecarcinus lateralis. Check marks associated with individual protein spots indicate proteins which changed significantly in abundance across the molt stages and exhibit a strong contribution towards the separation among molt stages displayed along the respective component axis (see Fig. 13B and Table 4). 

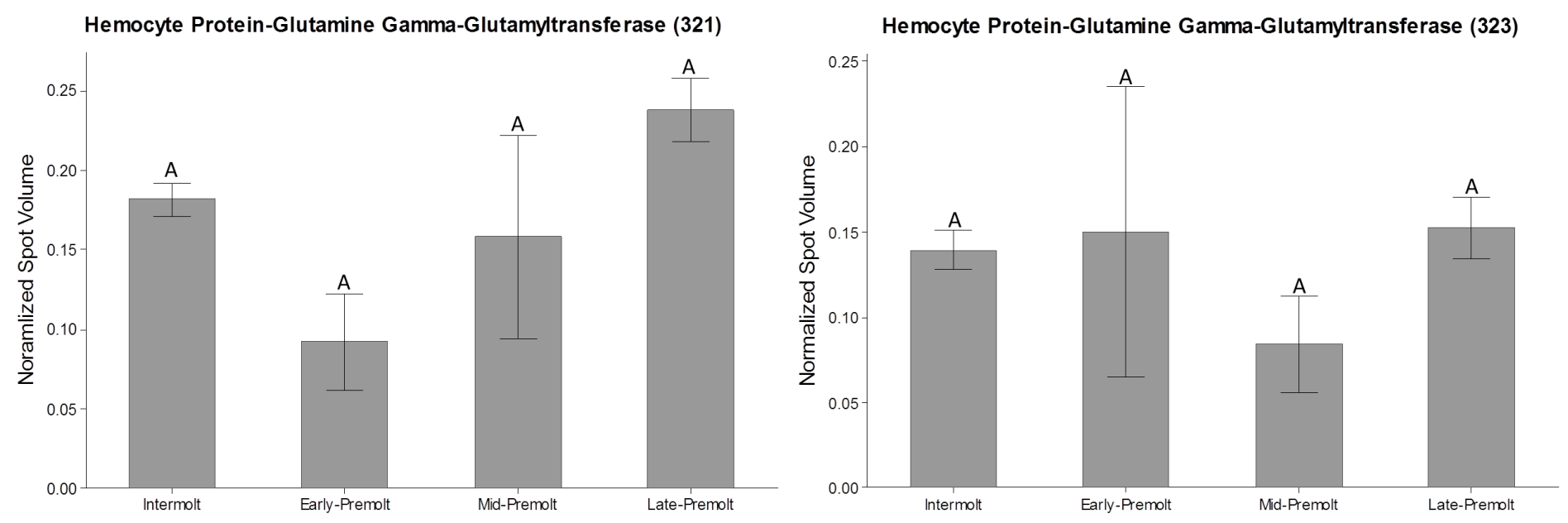

Transglutaminase (288)
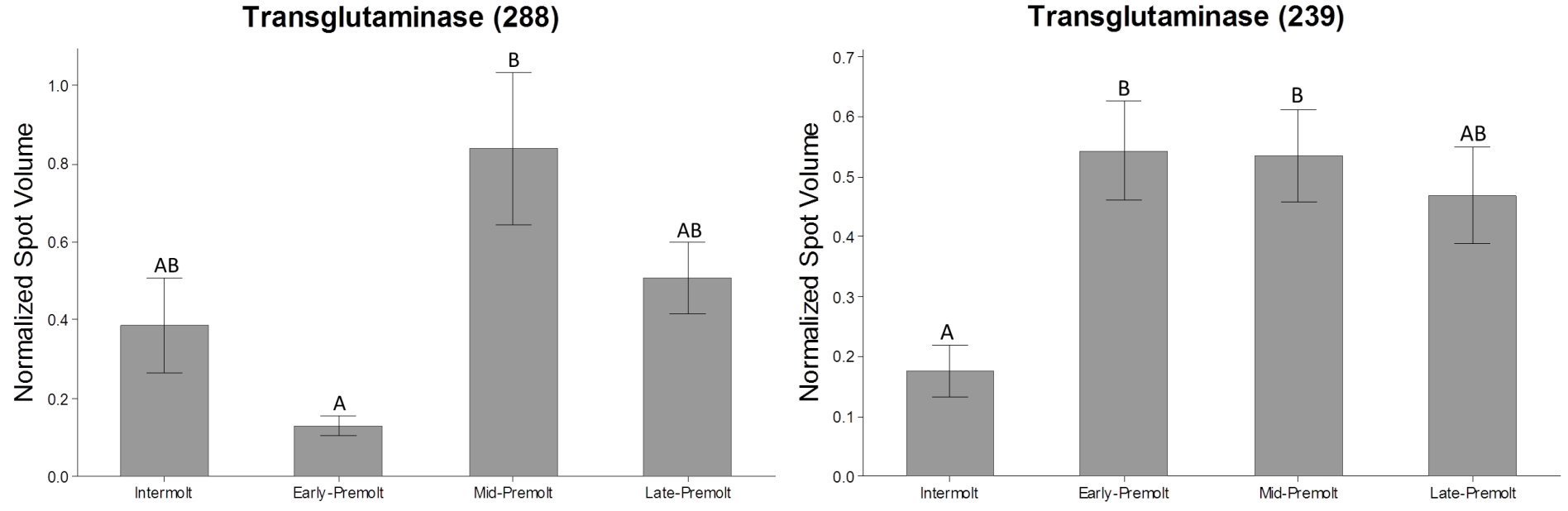

\section{Transglutaminase (237)}
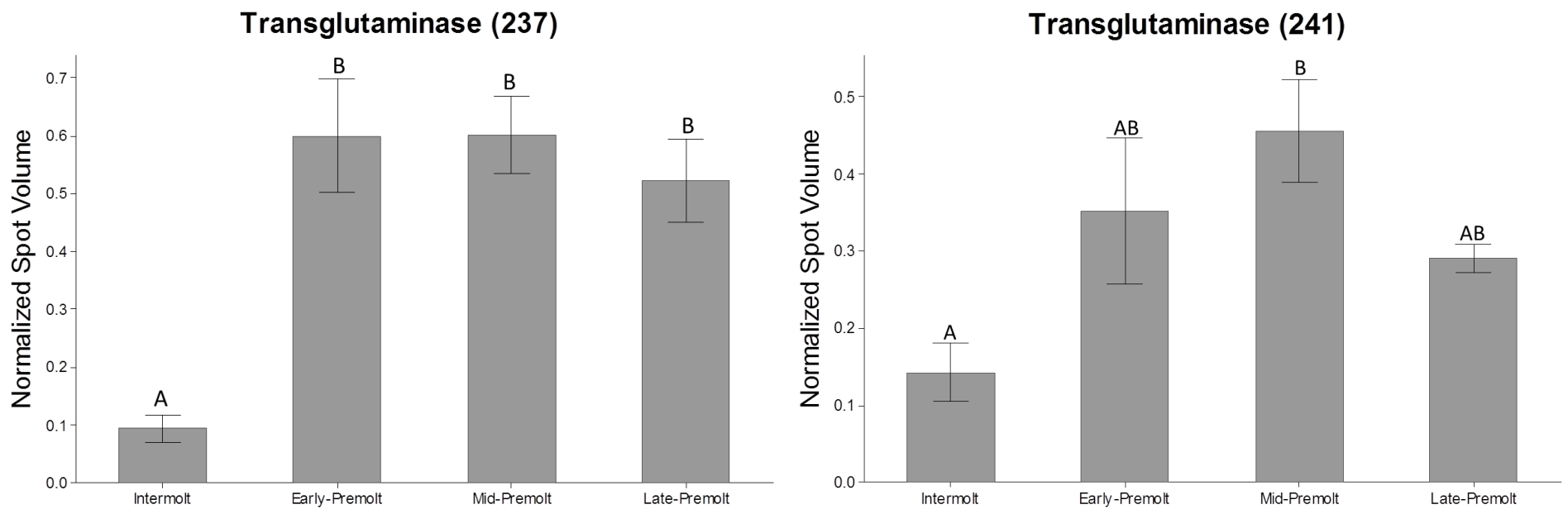

Figure 17. Levels of transglutaminase proteins in the YO of G. lateralis over the course of the molt cycle. Spot volumes were obtained by normalizing against the volume of all proteins and show means \pm s.e.m. $(\mathrm{N}=4)$. Molt stages that do not share a letter within individual graphs are significantly different (Tukey's HSD). Spot numbers are shown in parentheses. 
Six isoforms of transglutaminase were identified in the G. lateralis YO, two of which were distinctly identified as hemocyte proteins [(spots 321, 323) Fig. 16]. Distinct differences in both the molecular weight, as well as the abundance profiles (Fig. 17) of the hemocyte isoforms from the other four identified isoforms lead us to believe they are different orthologous homologs, specifically isoforms localized to hemocytes and the intracellular compartment, respectively.

Due to the multifaceted nature of this protein, it is difficult to attribute a specific role to it. In crustaceans, TGase has primarily been implicated in hemocyte-derived clotting and protein cross-linking reactions, as such, this section will primarily focus on the role of TGase in sclerotization of the exoskeleton. However, other possible roles of PTMs initiated by intracellular TGase in the cytoskeleton, energy metabolism, and oxidative-stress response, will be discussed further in their corresponding sections.

Although not significant, it is compelling to note that the hemocyte TGases show increased abundance in the intermolt and late premolt stages. The timing of this protein expression corresponds with previous discussion of sclerotization and melanization initiated by both hemocyanin and cryptocyanin. Expression of the crustacean clotting protein precursor [(spots 17, 418, 419) Fig. 16], a high density lipoprotein substrate for plasma clotting by TGase, was predominately identified in the early, mid, and late premolt stages. Presumably, these inverse expression profiles of hemocyte TGase and its substrate could be accounted for by synthesis of clotting protein precursors prior to their utilization during ecdysis. Such a preemptive synthesis of the clotting protein precursor would provide ready access to clotting mechanisms upon separation of the old exoskeleton from the freshly synthesized exocuticle, particularly in the extracellular matrix of internal organs. Microarray evidence for a 7.6 fold increase in clotting protein precursor transcript during late premolt of the blue crab, Portunus pelagicus, as well as other PO-activating 
transcripts such as antimicrobial proteins and hemocyanin, further support this notion (Kuballa and Elizur, 2008).

Taken together, the hemocyanin, cryptocyanin, and hemocyte TGase data yielded by this proteomic study provide supporting evidence and interesting insights into the complex physiology of ecdysis, particularly in regards to sclerotization and melanization.

\section{Cytoskeletal Proteins}

As a dynamic endocrine gland, the YO shows distinct histological changes with progression through the molt cycle. The intermolt $\mathrm{YO}$ is characterized by small cells, poor in cytoplasm, loosely organized in lobules with intra-lobular spaces (Buchholtz and Adelung, 1980; Smija and Sudha, 2016). As the molt cycle progresses to early premolt, YO cells become enlarged and are closely packed in lobules surrounded by large blood sinuses and fine capillaries. Observation of secretory vesicles is first noted towards the end of early premolt and increases through the mid premolt stage (Smija and Sudha, 2016). In particular, mid premolt cells adjacent to blood sinuses and capillaries accumulate large numbers of secretory vesicles, which seem to be released directly into the blood sinuses or capillaries (Smija and Sudha, 2016). Mid premolt YOs are further characterized by continued hypertrophy of the cells. Within the hypertrophied lobules of the early and mid premolt YO, dense amounts of smooth endoplasmic reticulum (SER), characteristic of the mammalian steroid secreting cells, are found in the cells of several individual lobules (Buchholtz and Adelung, 1980). The late premolt YO is slightly smaller in size and individual lobules are composed of a smaller number of cells than in mid premolt. Blood sinuses are large in this stage and intra-lobular spaces again become visible. Towards the end of the late premolt stage $\left(\mathrm{D}_{4}\right)$ the organ shows signs of degeneration (Smija and Sudha, 2016). 


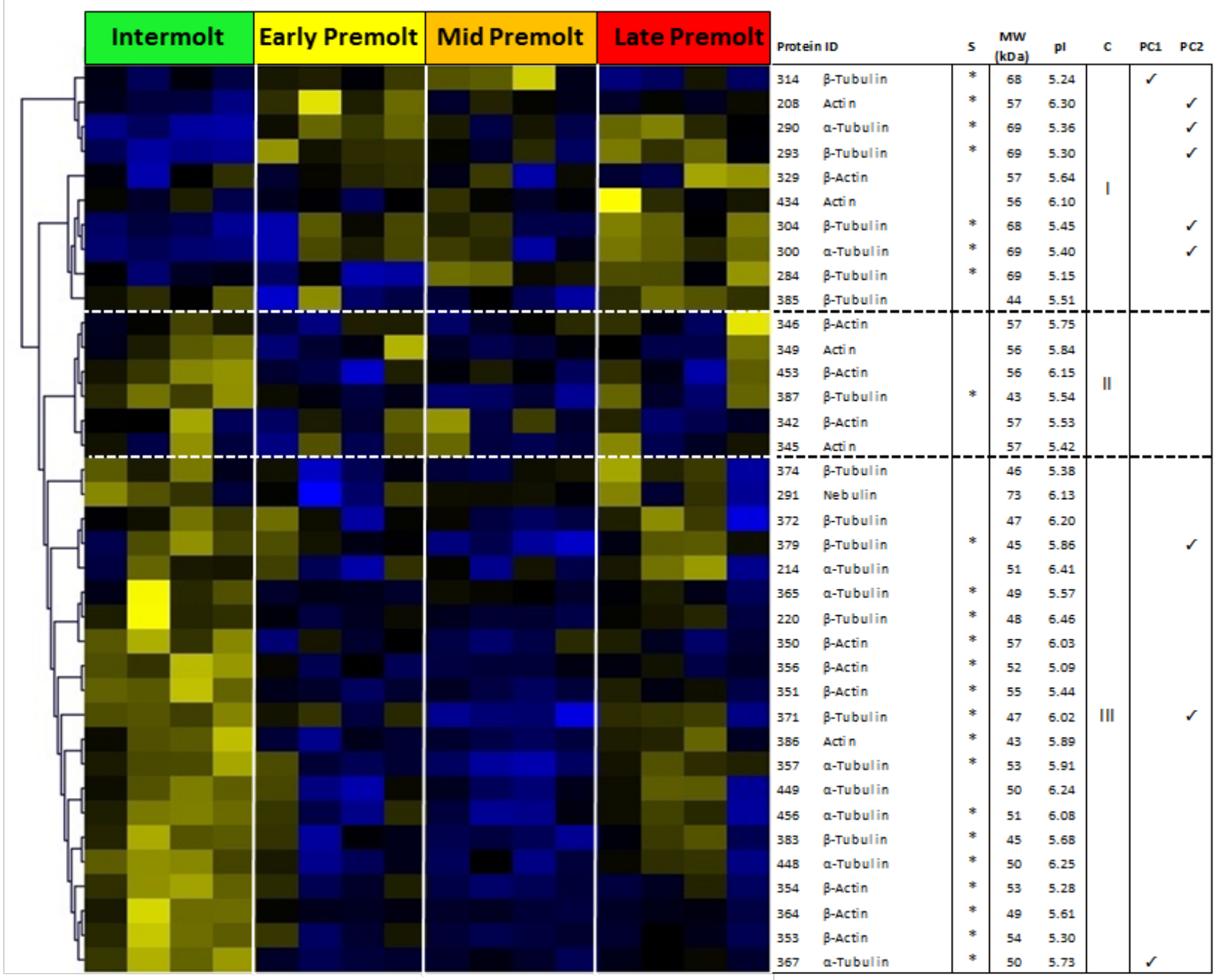

Abundance change:

Figure 18. Hierarchal clustering using Pearson's correlation depicting relative protein abundance (normalized spot volume) for all detected cytoskeletal proteins within the Y-organ of Gecarcinus lateralis. Check marks associated with individual protein spots indicate proteins which changed significantly in abundance across the molt stages and exhibit a strong contribution towards the separation among molt stages displayed along the respective component axis (see Fig. 13B and Table 4). 
The substantial histological changes of the YO incurred by the molt cycle indicate a need for structural re-organization of the individual cells, as well as the endocrine gland as a whole. Cytoskeletal proteins, such as actin and tubulin, are ubiquitously expressed in all cell types and are among the most abundant proteins present within the cell. Thus, the six isoforms of actin and tubulin corresponding to Cluster II (Fig. 18) most likely represent constitutively expressed cytoskeletal elements necessary for basic cellular functions. Moreover, the significant number of tubulin and actin proteins found to be increased in abundance in intermolt and late premolt (Cluster III) are also presumably characteristic of those cytoskeletal elements necessary for basic cellular function. The small cell size of the basal YO in intermolt, and the degenerating cells in late premolt, likely are predominantly composed of cytoskeletal elements, as the main endocrine function of the gland is depressed during these stages. Ten isoforms of cytoskeletal proteins constitute Cluster I and are primarily expressed in the early, mid, and late premolt stages. Subtle changes in abundance of these isoforms across the three premolt stages seem to set forth an interesting narrative, particularly as four tubulins and one actin isoform contribute significantly to separation of the premolt stages along PC2.

Following the histological changes across the molt cycle, the increase in actin and tubulin isoforms in early premolt correspond with an increase in cell size and vascularization, as well as the formation of secretory vesicles. The observed increases in average protein abundance for these cytoskeletal isoforms would be necessary to induce such histological changes. Increases in cell size, vascularization, and formation of secretory vesicles continue in mid premolt. However, the average abundance for several actin and tubulin isoforms in this stage is somewhat less than that observed in early and late premolt. While this seems counterintuitive, a plausible explanation involves stabilization of microtubules and actin through PTMs, especially considering the 
relatively higher molecular weight of tubulin proteins in Cluster I relative to Clusters II and III. Circling back to the TGases introduced in the previous section, polyamination of tubulin has been shown to play an important role in the stabilization of microtubule and actin filaments (Lai et al., 2017; Terman and Kashina, 2013). The four presumably intracellular TGases identified (Fig. 16) were increased in average abundance in early and mid premolt, with one isoform in particular increased only in mid premolt and contributing to the separation of mid premolt along PC2. Finally, as the late premolt stage is reached the YO is characterized by degeneration in cell size and function. A corresponding decrease in TGase suggests destabilization of actin and tubulin filaments, resulting in the breakdown of long fibers into individual subunits as the cells shrink in size. Two likely explanations for the precise intracellular roles of these cytoskeletal elements are laid out below.

I. Vesicular secretion of ecdysteroids. Contrasting with the long-held simple-diffusion paradigm of steroid secretion, evidence for vesicle-mediated secretion of ecdysteroids has been increasing over the years. Identification of secretory vesicles within the YO were first noted in the green crab, Carcinus maenas, in 1975 (Chassard-Bouchaud and Hubert, 1975). Since, secretory granules originating from the SER have been identified in the YO of many malacostracans and thoracic gland of many insects (Buchholtz and Adelung, 1980; Birkenbeil, 1983; Babu et al., 1989; Yamanaka et al., 2015; Smija and Sudha, 2016). Due to the more hydrophilic nature of ecdysone relative to other commonly studied steroids such as progesterone, testosterone, and estradiol, it is much more likely to require active transporters to traverse lipid bilayers (Yamanaka et al., 2015). Pertinently, Rab-GDP Dissociation Inhibitor (Rab-GDI; spot 332) is not only increased in abundance during mid premolt, but contributes to the distinctive separation of this stage from the 
early and late premolt stages along PC2. Rab-GDIs increase recycling of vesicular transport molecules, thus allowing for increased vesicular transport within cells.

II. Trafficking of steroid precursors. The vertebrate cytoskeleton has been shown to play an essential role in intracellular trafficking of cholesterol to the mitochondria to initiate metabolic steroid pathways (Hall et al., 1979). $\beta$-tubulin and microtubule formation have been shown to play a significant role in prothroacicotropic hormone (PTTH) stimulated ecdysteroidogenesis in the prothoracic gland of the tobacco hornworm, Manduca sexta (Rybczynski and Gilbert, 1995; Watson et al., 1996).

While increases in abundance of cytoskeletal elements such as tubulin and actin alone cannot provide direct evidence for or against either hypothesis laid out above, it does suggest a greatly important role in cytoskeletal organization of the YO to function as an endocrine gland. Previous proteomic analysis of the $C$. maenas YO across the molt cycle provides corroborating evidence for cytoskeletal rearrangement associated with periods of ecdysteroid synthesis and secretion (Hamer, 2015).

\section{Immune Response}

Due to the increasingly profitable aquaculture industry for shrimp, the immune response of decapod crustaceans to various bacterial and viral challenges has become a topic of interest in the field of invertebrate biology. The main functions of the innate crustacean immune response are involved with (i) hemocyte and hematopoiesis, (ii) prophenoloxidase activation, and (iii) lectins and pattern recognition (Cerenius et al., 2010).

Similar to previously described patterns of change in protein abundance, two distinct clusters of immune-related proteins were observed (Fig. 19). Cluster I represents those nine identified proteins which increase in abundance during the premolt stages when the $\mathrm{YO}$ is active 
and the organism is preparing for ecdysis. Cluster II represents seven identified immune protein isoforms that are most abundant in the late premolt and intermolt stages, which correspond to periods of glandular atrophy specifically within the YO, but increased immune response within the organism as a whole. Discussion of the immune response in this section will focus specifically on prostaglandin-related proteins and transferrins; for discussion on the clotting protein precursors please refer to the previous section regarding transglutaminase activity.

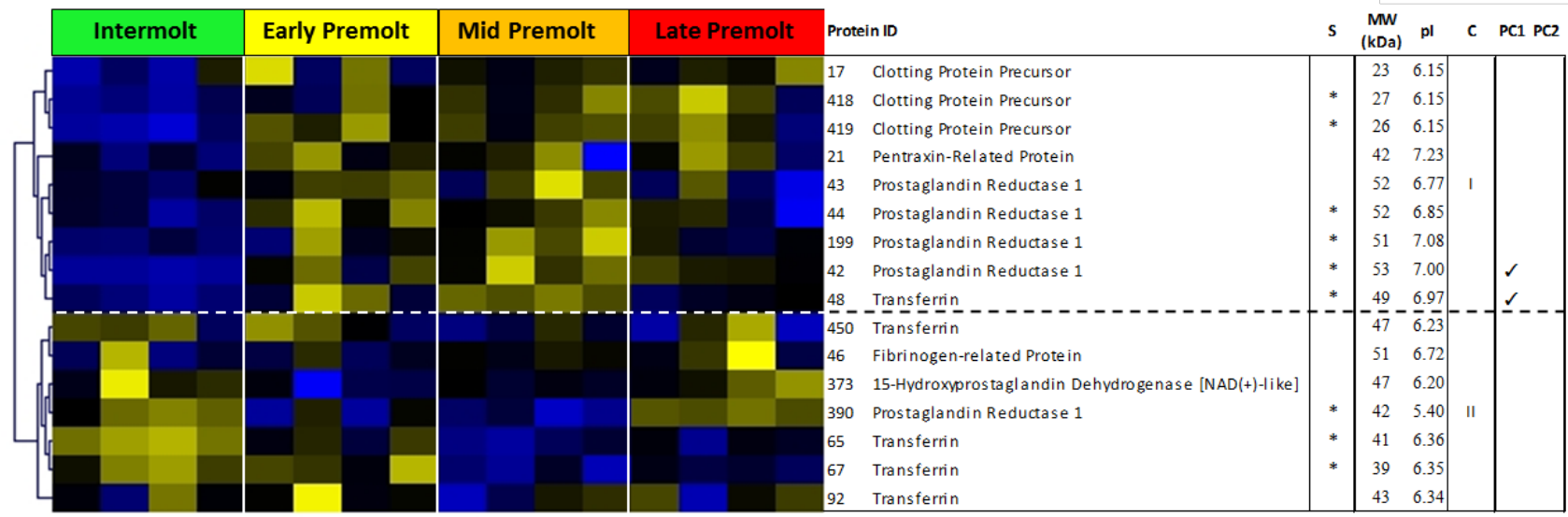

Abundance change: $-1.99$ $-0.14$ 2.70

Figure 19. Hierarchal clustering using Pearson's correlation depicting relative protein abundance (normalized spot volume) for all detected immune proteins within the Y-organ of Gecarcinus lateralis. Check marks associated with individual protein spots indicate proteins which changed significantly in abundance across the molt stages and exhibit a strong contribution towards the separation among molt stages displayed along the respective component axis (see Fig. 13B and Table 4). 
Prostaglandins are eicosanoid compounds synthesized from the metabolism of polyunsaturated fatty acids. Within invertebrates prostaglandins have been shown to be involved with vitellogenesis as well as mediators of inflammation and hemocyte recruitment (Cerenius et al., 2010; Prasertlux et al., 2011; Rowley et al., 2005). Two identified prostaglandin-related genes were identified in the G. lateralis YO, prostaglandin reductase and 15-hydroxyprostaglandin dehydrogenase, both of which inactivate prostaglandins through catabolism into metabolites (Prasertlux et al., 2011). Prostaglandin reductase 1 has also been shown to have catabolic effects on the inflammatory mediator leyukotrine $\mathrm{B}_{4}\left(\mathrm{LTB}_{4}\right)$, providing further inhibition of an inflammatory response (Yokomizo et al., 2010). Notable differences in both the molecular weight and $\mathrm{pI}$ of the observed prostaglandin inactivators suggest PTM-modulated activation and inactivation during specific periods of the molt cycle. Based on previous studies of the crustacean immune response over the molt cycle, total hemocyte count, and thus innate immune response, has been shown to be the lowest during intermolt and increases steadily to reach highest hemocyte levels during postmolt (Cheng and Chen, 2002). Specifically, hemocyte recruitment to the epidermis during premolt and ecdysis is vital as detachment from the old cuticle allows for increased bacterial penetration (Cheng and Chen, 2002).

Transferrins are a class of iron-binding glycoproteins shown to play a role in the innate immune response through sequestration of iron, thus impeding bacterial survival (Smith and Chisholm, 2001). Interestingly, pacifastin, an inhibitor of phenoloxidase-activating serine proteases, contains 3 lobes of conserved transferrin domains. The highly conserved sequence of these transferrin domains indicate that pacifastin retains the iron-binding capacity of other transferrin proteins (Liang et al., 1997). Five identified isoforms of transferrin were identified with varying expression patterns in the G. lateralis YO. Strikingly, four of the five isoforms appear to 
be most abundant during the intermolt stage, possibly indicative of regulation of phenoloxidase activity.

Briefly, pentraxins are calcium-dependent enzymes involved in humoral innate immunity. Specifically, a C-reactive protein (CRP) was identified as a component of hemolymph in the horseshoe crab in 2002; the first identified pentraxin in invertebrates (Tharia et al., 2002). CRPs are typically expressed in response to tissue injury and promote phagocytosis of bacteria and activation of the complement pathway. Increased premolt abundance of the pentraxin-related protein identified in this study corresponds with the timing of other increased immune responses associated with secretion of a new exoskeleton and separation from the old cuticle.

\section{Energy Metabolism}

Steroidogenesis and changes in metabolic pathways such as glycolysis and the citric acid cycle (TCA) are closely linked. As such, continuous changes in energy metabolism pathways were observed as the YO transitioned through the discrete stages of the molt cycle. Five clusters of proteins were detected based upon similarities in protein abundance among each of the molt stages (Fig. 20). These changes in metabolic pathways represent the cellular foundation which supports ecdysteroidogenesis in the molting gland. For clarity, the observed changes in energy metabolism proteins will be discussed by energetic pathway.

Glycolysis

The premolt YO (Clusters I and IV) is distinctly characterized by increased abundance of many glycolytic enzymes, owing to the energy demand of ecdysteroid synthesis and glandular hypertrophy. To begin simply, glycolytic enzymes triosephosphate isomerase (TPI), glyceraldehyde 3-phosphate dehydrogenase (GAPDH), and phosphopyruvate hydratase (enolase) were all abundantly expressed in the early, mid, and late premolt YO. Such upregulation of 
glycolytic enzymes in the premolt stages confirms the notion of an increased energetic demand to support protein synthesis, cell growth, and most importantly, ecdysteroidogenesis.

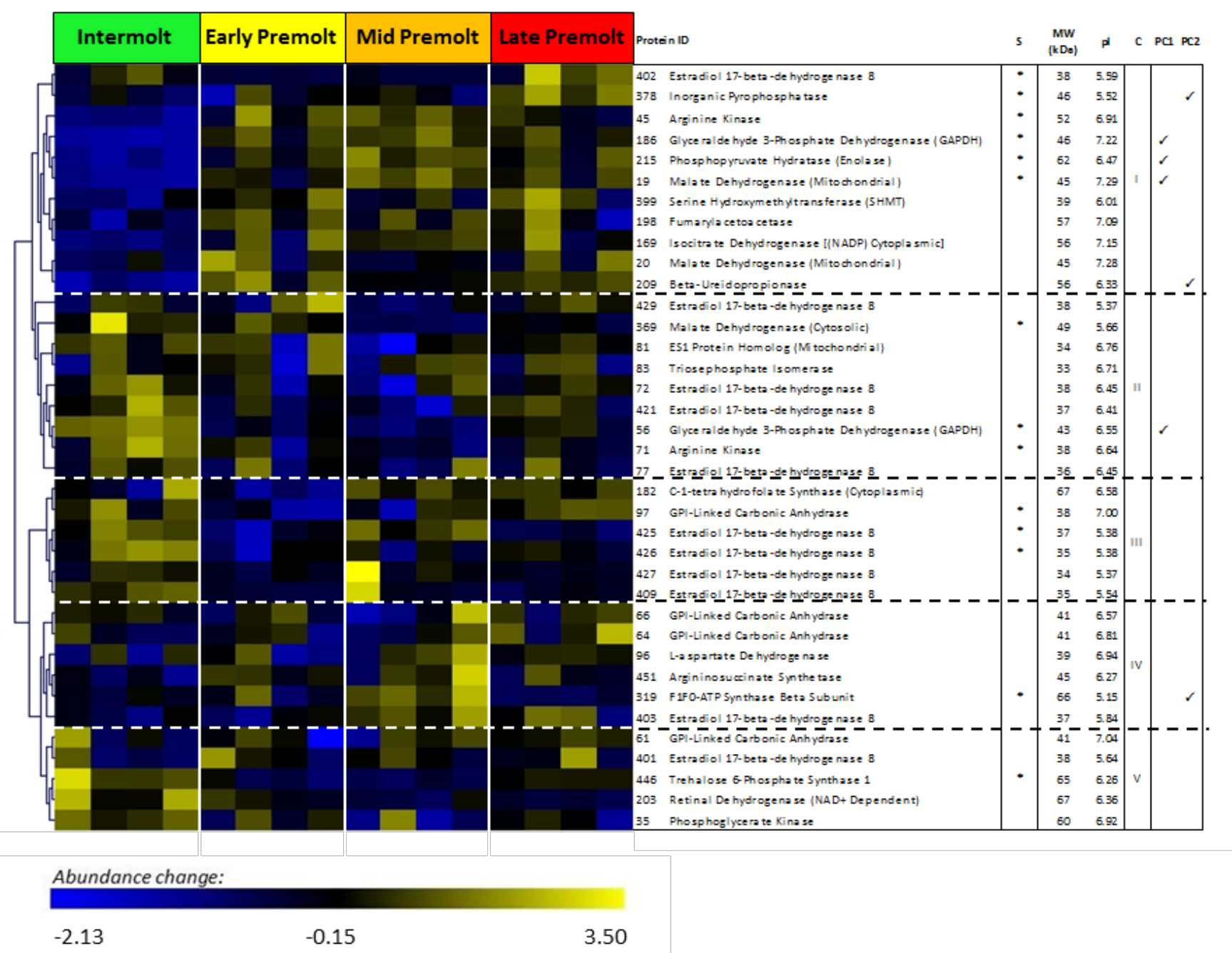

Figure 20. Hierarchal clustering using Pearson's correlation depicting relative protein abundance (normalized spot volume) for all detected energy metabolism proteins within the Y-organ of Gecarcinus lateralis. Check marks associated with individual protein spots indicate proteins which changed significantly in abundance across the molt stages and exhibit a strong contribution towards the separation among molt stages displayed along the respective component axis (see Fig. 13B and Table 4). 
The glycolytic pathway has several branching points from which intermediates can feed into other biosynthetic pathways (Fig. 22). Changes in the abundance or activity of glycolytic enzymes can drive reactions toward or away from these branching pathways. For example, TPI is responsible for the conversion of dihydroxyacetone phosphate to glyceradehyde-3-phosphate (G3P), the substrate for GAPDH. Changes in TPI activity are associated with changes in glycolytic rates. More specifically, decreases in TPI activity lead to a buildup of early glycolytic intermediates, and can drive the reactions towards other biosynthetic pathways such as the pentose phosphate pathway (PPP) and the hexosamine pathway (Chandel, 2015). The PPP leads to synthesis of nucleotides, glycogen, and NADPH, which is essential for antioxidant pathways. The hexosamine pathway synthesizes glycoproteins, such as chitin in insects and crustaceans (Tomiya et al., 2006). While TPI was expressed in all molt stages, the overall pattern of abundance within each stage suggest that acute changes in TPI expression may be used to drive reactions toward the branching biosynthetic pathways of glycolysis as needed.

It is important to note that while the expression of particular isoforms of the aforementioned proteins seem to be more abundant in the premolt stages, certain glycolytic enzymes, such as GAPDH and phosphoglycerate kinase, were also present within the intermolt YO. Basal levels of ecdysteroidogenesis and secretion continue in the intermolt YO, reaching hemolymph titers of 2-10 pg/ $\mu \mathrm{l}$ (Covi et al., 2010), thus requiring sustenance by basal metabolic rates.

\section{The Citric Acid Cycle}

The citric acid cycle is a vital biosynthetic pathway, both for ATP synthesis, but also metabolic intermediates for gluconeogenesis, lipid, amino acid, purine, and pyrimidine synthesis among others (Chandel, 2015). The cells of the YO are rich in mitochondria to support ecdysteroid 
synthesis, thus the identification of several mitochondrial related proteins, such as those involved in the TCA cycle, came as no surprise.

Intermediates of several energy metabolism pathways are interrelated. Important to the proper functioning of glycolysis is the 'malate-aspartate shuttle', by which malate transports electrons across the inner mitochondrial membrane and regenerates mitochondrial NADH for the electron transport chain and cytosolic NAD+ for glycolysis [Fig. 21; (Chandel, 2015)]. NADH produced during glycolysis is used to reduce malate in the intermembrane space of the mitochondria, which is then transported into the matrix via a malate/ $\alpha$-ketoglutarate $(\alpha-K G)$ antiporter. Within the matrix, NADH is regenerated by malate dehydrogenase (MDH), and the resulting oxaloacetate is converted to aspartate by L-aspartate dehydrogenase. Aspartate is then shuttled back to the intermembrane space through an aspartate/glutamate antiporter, converted back to malate, and the cyclic process continues. Beyond the malate-aspartate shuttle, MDH is important for the interconversion of malate and oxaloacetate in the TCA cycle. Increased abundance of both malate dehydrogenase and L-aspartate dehydrogenase are a product of the increased energy demand of the active premolt YO.

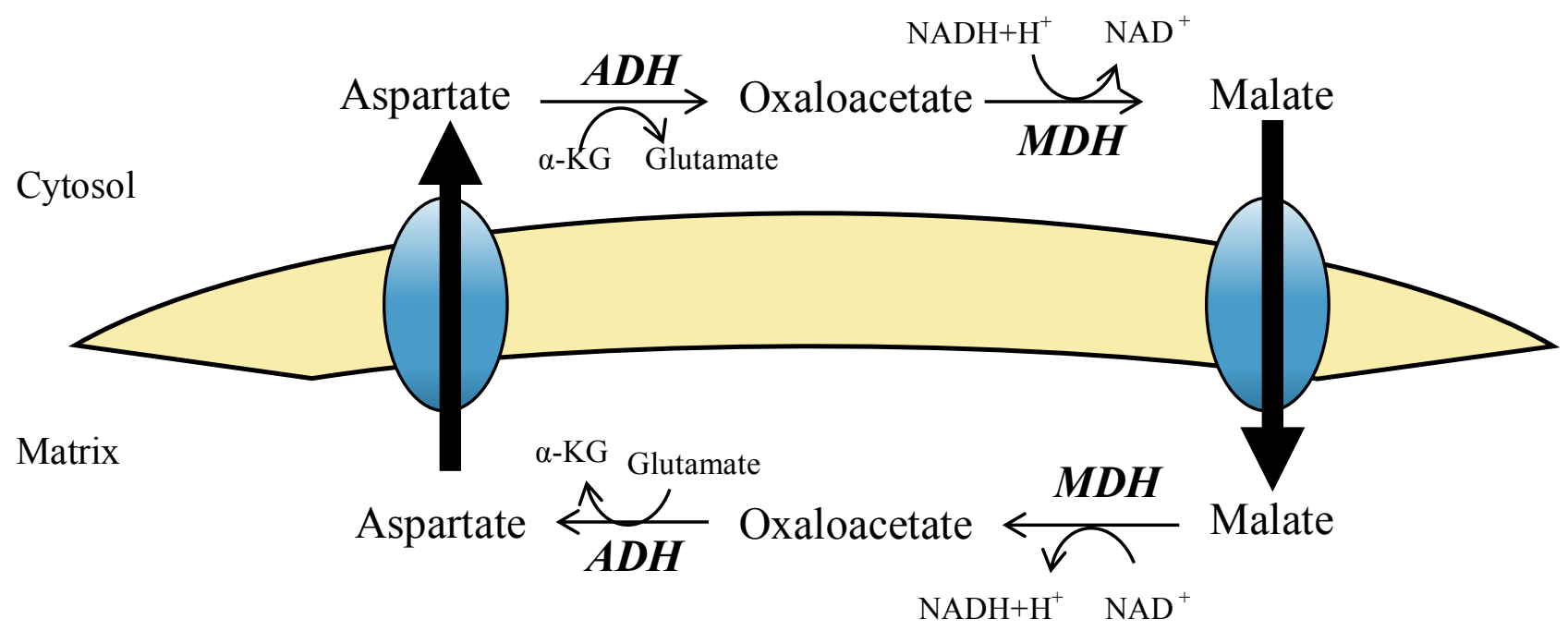

Figure 21. The malate-aspartate shuttle of the mitochondria. Malate is shuttled into the matrix of the mitochondria to regenerate reducing equivalents which supply the electron transport chain. Malate is subsequently converted to aspartate, and shuttled back into the cytosol. The conversion of aspartate to malate within the cytosol regenerates $\mathrm{NAD}^{+}$which serve to maintain glycolysis. 
Isocitrate dehydrogenase catalyzes the irreversible synthesis of $\alpha$-ketoglutarate from isocitrate. $\alpha \mathrm{KG}$ has been linked with the activation of mTOR, a vital protein complex in the activation and proliferation of the YO (Durán and Hall, 2012; Chang and Mykles, 2011). Proteomic analysis of the C. maenas YO suggests nutrient-sensing activation of the YO whereby increased glutaminolysis in the premolt YO would lead to an increase in intracellular $\alpha$ ketoglutarate, feeding into the TCA cycle (Hamer, 2015). Transglutaminase has been shown to inactivate both $\alpha$-ketoglutarate dehydrogenase and GAPDH through crosslinking with polyglutamine residues (Cooper et al., 1997). Taken together, these data may indicate a selfregulating metabolic checkpoint within the $\mathrm{YO}$ where glutaminolysis during periods of high nutrient availability, such as premolt, feeds into the TCA cycle, and prevents TGase inactivation of GAPDH and $\alpha-\mathrm{KG}$ dehydrogenase through the catabolism of these metabolic intermediates.

Synthesis of purines, pyrimidines, and amino acids are vital in the activation of the YO for the hypertrophy and protein synthesis that ultimately supports ecdysteroidogenesis. Beyond the functions already discussed, several of the identified TCA enzymes also feed into the biosynthetic pathways of amino acid and nucleotide synthesis. Increased activity of malate dehydrogenase leads to increased oxaloacetate, which can then be used for the synthesis of aspartate by L-aspartate dehydrogenase. Subsequently, aspartate can be used for purine and pyrimidine synthesis. Additionally, glutamate, which can be synthesized from $\alpha \mathrm{KG}$, can be further metabolized into glutamine, proline, and arginine. These two pathways are highly interrelated- the transamination of aspartate and $\alpha \mathrm{KG}$ results in oxaloacetate and glutamate, a reaction that can be favorably reversed depending on substrate concentrations (Chandel, 2015). Increased abundance of the key enzymes regulating these pathways in the premolt stages are indicative of the high-energy requirements of steroidogenic cells. 


\section{ATP Synthesis}

Expression of the previously discussed glycolytic and TCA enzymes in all three premolt stages, while abundant expression of $\mathrm{F}_{1} \mathrm{~F}_{0}$ ATP synthase was only identified in the mid premolt YO, provides further evidence for the use of branching biosynthetic pathways from both glycolysis and the TCA cycle. These branching points likely proceed in the anabolic direction in early premolt, providing building blocks essential for the active YO. Contrastingly, the reverse, catabolic reactions of these branching pathways would be favored in the degenerating YO of late postmolt.

\section{Amino Acid Metabolism}

Argininosuccinate synthetase (ASS), predominately upregulated in mid premolt, is an important enzyme in the urea cycle and is linked to the TCA cycle through common metabolic intermediates. Argininosuccinate is generated in the cytosol by ASS, which combines citrulline with a nitrogen atom from aspartate. Subsequent reactions produce arginine which is then converted to urea (Morris, 2002). While nitrogenous excretion from G. lateralis primarily occurs through ammonia excretion through the branchial chamber (Wolcott, 1991), the urea cycle may play a role in amino acid metabolism associated with protein turnover. Of importance are the metabolic intermediates aspartate, fumarate, and malate which link the urea cycle to the TCA cycle (Fig. 22).

Arginine kinase is yet another important enzyme involved in the metabolism of arginine, glutamate, and proline. As previously discussed in detail with the TCA cycle, several enzymes which synthesize precursors of arginine and proline were upregulated in the premolt $\mathrm{YO}$, alluding to the importance of amino acid metabolism and ultimately protein turnover. While this metabolic pathway can easily explain the increased abundance of one isoform of arginine kinase (spot 45) in 


\section{GLYCOLYSIS}

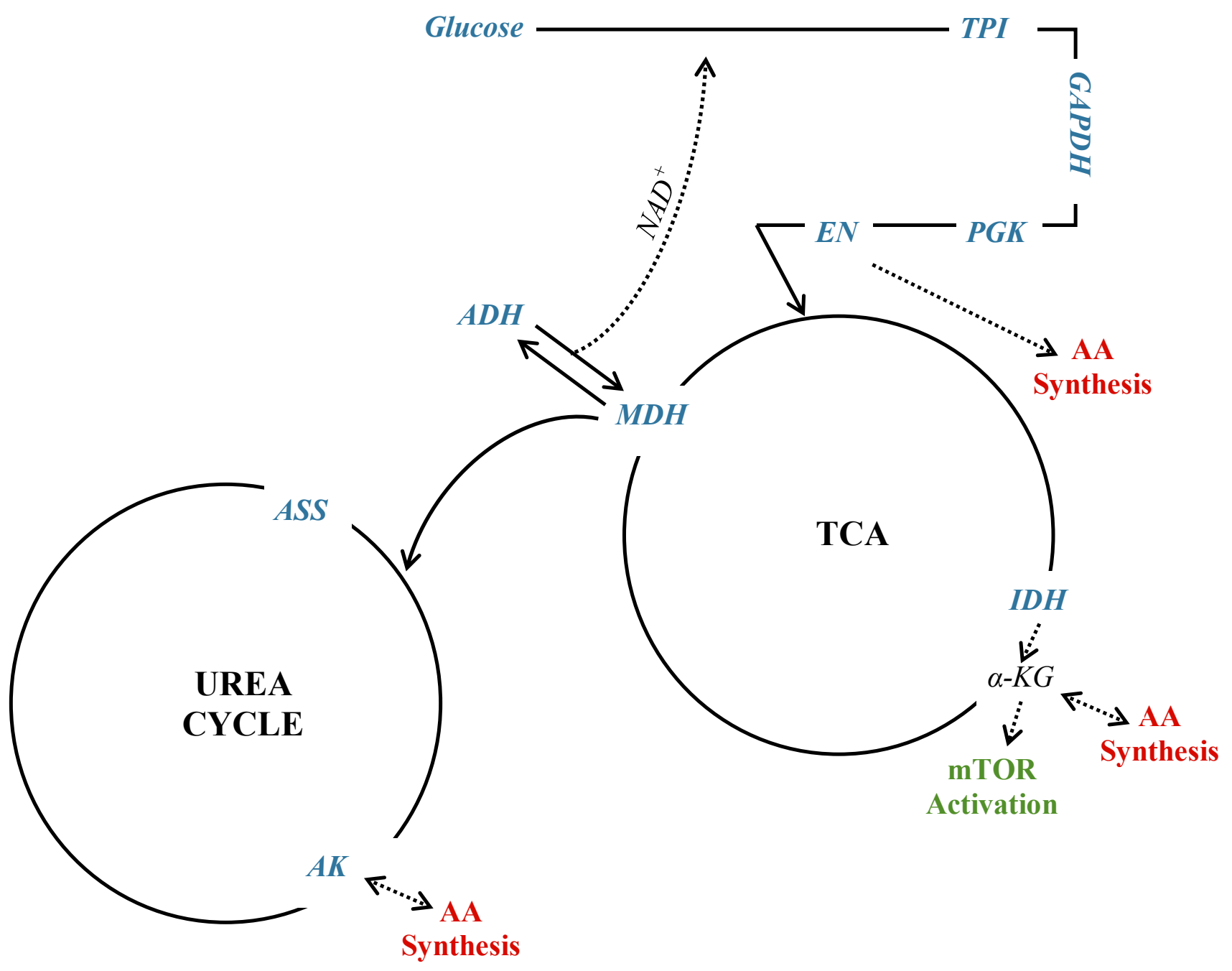

Figure 22. Schematic representation of the major energetic enzymes identified within the YO of $G$. lateralis. Identified enzymes are indicated in blue, points of amino acid synthesis are indicated in red, and mTOR activation is indicated in green. Abbreviations are as follows: ADH, aspartate dehydrogenase; AK, arginine kinase, ASS; argininosuccinate synthetase; EN, enolase;GAPDH, glutamate dehydrogenase; IDH, isocitrate dehydrogenase; $\mathrm{MDH}$, malate dehydrogenase; PGK, phosphoglycerate kinase; TPI, triosephosphate isomerase.

the energy-demanding premolt stages, another isoform (spot 71) appears to be highly upregulated in the basal intermolt stage. Arginine kinase may play multiple roles within the YO depending on 
the molt stage during which it is expressed. MIH inhibition of ecdysteroidogenesis in the YO involves a 'summation' phase in which nitric oxide synthase (NOS) is activated, and nitric oxide (NO) acts as a second messenger by means of increasing cGMP (Chang and Mykles, 2011). Larginine and NADPH are used by NOS to produce NO and another familiar product, citrulline (Chandel, 2015).

\section{One Carbon Metabolism}

One-carbon metabolism is necessary for regeneration of NADPH, an important molecule in the biosynthesis of fatty acids, amino acids and nucleotides. The necessity of one-carbon metabolism in proliferating tumor cells highlights the importance of this pathway for cell growth such as that which occurs in the early and mid premolt YO. Again tying in glycolytic intermediates, 3-phosphoglycerate initiates the one-carbon metabolic pathways involving serine and glycine interconversion. Conversion of glutamate to $\alpha \mathrm{KG}$ by phosphoserine aminotransferase leads to the generation of serine which can then be used for protein synthesis as well as the serine-glycine shuttle which regenerates NADPH. The enzyme serine hydroxymethyltransferase (SHMT) interconverts serine and glycine, using tetrahydrofolate as a cofactor. 5,10-methylene tetrahydrofolate can then be used to regenerate NADPH in the cytosol, the subsequent product is shuttled into the mitochondria and again used as a cofactor in the regeneration of NADPH and interconversion of serine and glycine (Chandel, 2015). Synthesis of tetrahydrofolate by C-1tetrahydrofolate synthase in the YO supports serine and glycine synthesis, as well as NADPH regeneration which is used to support subsequent generation of macromolecules.

\section{Estradiol 17- $\beta$ Dehydrogenase}

A staggering eleven isoforms of estradiol 17- $\beta$ dehydrogenase were identified in the YO, with expression of multiple isoforms present in each molt stage. While this enzyme has been shown 
to be crucial in vitellogenesis and ovary maturation in female crabs (Thongbuakaew et al., 2016), its possible role in the male YO has yet to be elucidated. Due to the highly analogous structures of estradiol and ecdysteroids, it seems plausible that estradiol $17-\beta$ dehydrogenase could function in ecdysteroidogenesis, thus explaining the abundance throughout each molt stage. A previous study on mammalian estradiol $17-\beta$ dehydrogenase revealed that the enzyme also has $20 \alpha-$ hydroxysteroid dehydrogenase activity, providing further reason to believe that this enzyme could be implicated in previously uncharacterized reactions of ecdsysteroid metabolism (Strickler and Tobias, 1980).

\section{Other Energetic Pathways}

Trehalose is a non-reducing disaccharide that acts as an energy source, usually in addition to that provided by glucose, in prokaryotes, yeast, plants, and invertebrates (Chung, 2008). This sugar has been implicated in adaptation to extreme environmental stress, particularly desiccation. A previous study of the blue crab, Callinectes sapidus, has revealed gene expression of trehalose 6-phosphate synthase (TPS) in the YO among several other organs. Characterization of TPS activity in hemocytes revealed that enzyme activity and hemolymph titers were highest in postmolt. These postmolt increases in trehalose were in response to the significant stress involved with ecdysis, particularly as increased water uptake post-ecdysis can lead to disruption of ion balance (Mykles, 1980). Arguably, the observed increase in abundance of TPS in the intermolt YO may be reflective of a switch to trehalose to support ecdysis and the subsequent postmolt physiology of crustaceans.

\section{Protein Homeostasis}

A quick glance at the hierarchical clustering of proteins involved in proteostasis of the YO reveals several complex, yet interrelated pathways that appear to be dependent upon the molt cycle 
(Fig. 23). Protein homeostasis within cells is primarily regulated by two classes of proteins: (i) molecular chaperones which aid in stabilization and folding of proteins, and (ii) proteases which aid in degradation of proteins and can lead to the recycling of amino acids.

To begin, Cluster I represents proteins primarily involved in proteostasis of the premolt YO. Both $\alpha$-L-fucosidase and $\beta$-hexosaminidase are members of the glycosidase family of enzymes involved in degradation of glycolipids and glycoproteins (Reglero and Cabezas, 1976; Intra et al., 2008). $\beta$-hexosaminidase has been specifically implicated in the degradation of the chitin exoskeleton in both crustaceans and insects (Tomiya et al., 2006). It is likely that identification of this protein in the $\mathrm{YO}$ is an artifact of the hemolymph, and its expression corresponds with expected increases in chitin metabolism during premolt. Prenylcysteine oxidase (PCL), is a FAD-dependent monoxygenase involved in lysosomal degradation of lipid-modified proteins (Lu and Hoffman, 2006). Increased abundance of these catalytic enzymes may indicate an increase in protein turnover during the metabolically-demanding premolt stages of ecdysteroidogenesis. 


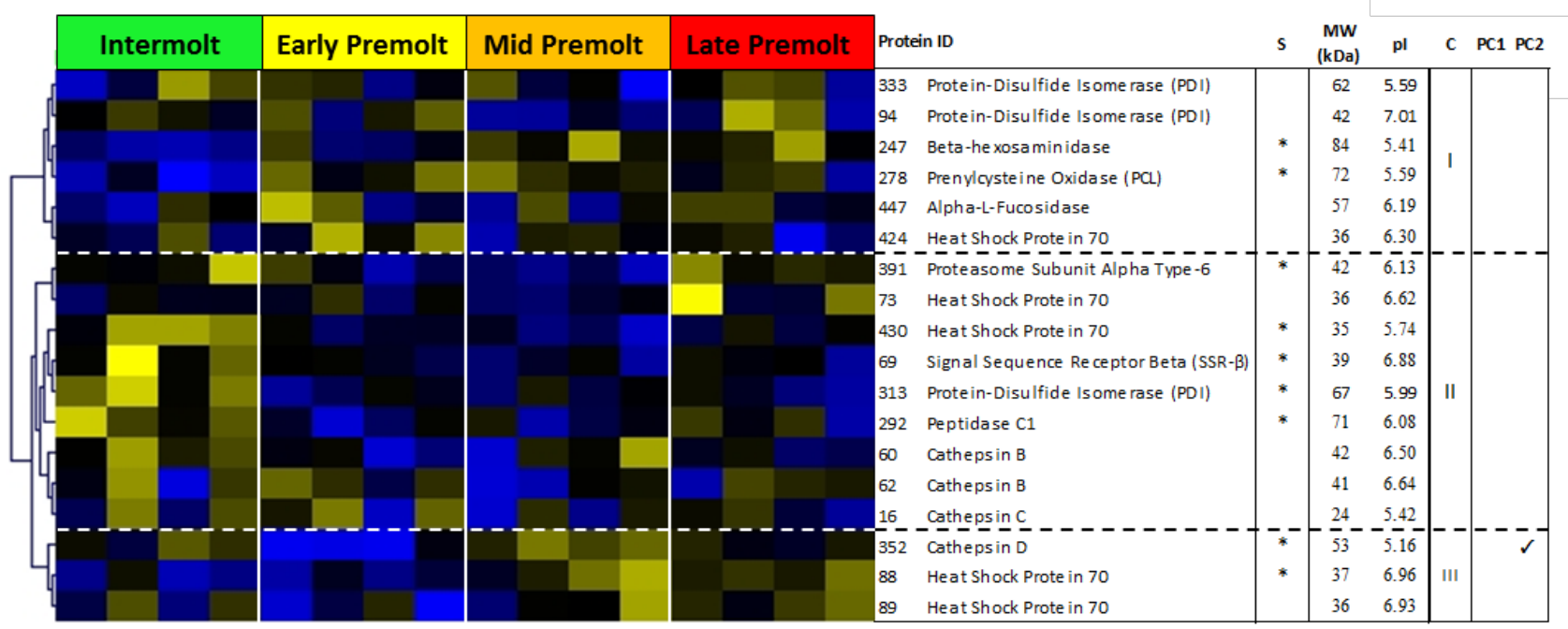

\section{Abundance change:}

Figure 23. Hierarchal clustering using Pearson's correlation depicting relative protein abundance (normalized spot volume) for all detected proteins involved in protein homeostasis within the Yorgan of Gecarcinus lateralis. Check marks associated with individual protein spots indicate proteins which changed significantly in abundance across the molt stages and exhibit a strong contribution towards the separation among molt stages displayed along the respective component axis (see Fig. 13B and Table 4).

The intermolt and late premolt stages (Cluster II) are characterized by expression of proteolytic enzymes connected to lysosomal (autophagy) and ubiquitin-proteasome protein degradation involved with atrophy of the YO. The proteasome subunit $\alpha-6$ (EC:3.4.25.1) forms a proteasome complex which participates in degradation of ubiquitinated proteins. Cathepsins B and $\mathrm{C}$, as well as peptidase $\mathrm{C} 1$ are cysteine proteases which catalyze the hydrolysis and subsequent degradation of many proteins, playing a vital role in protein and organelle turnover (Qui et al., 2008; Muller et al., 2012). Cathepsins B, D, and L have also been linked to regulating apoptosis in vertebrate immune systems via mitchondrial ROS-induced feedback to the lysosome which stimulates release of apoptotic factors (Cornus et al., 2010). Identification of abundant apoptosis 
inducing factor-3 (spot 337) in the intermolt YO was found to contribute significantly to intermolt separation from the premolt YOs along PC1. This identification is suggestive of a similar cathepsin-mediated apoptotic pathway in invertebrates, particularly in relation to YO atrophy.

Protein disulfide isomerase (PDI) is important in the formation of disulfide bridges in nascent polypeptides being synthesized within the ER. The subsequent reoxidation of PDI results in the transfer of electrons to $\mathrm{FAD}+$. The FADH generated in this reaction reacts with oxygen to produce $\mathrm{H}_{2} \mathrm{O}_{2}$, which must later be scavenged by antioxidant molecules (Chandel, 2015; Tomanek, 2015). Thus, PDI is not only an important enzyme in the maturation of proteins from the ER, but also the redox state of cells. Given the high rates of protein turnover occurring during the molt stages, the pattern of PDI upregulation exhibited in this context may represent increases in protein synthesis associated with ecdysteroidogenesis

Finally, four isoforms of heat shock protein 70 (HSP 70) were dispersed throughout the three clusters, representative of the importance of this molecular chaperone on proteostasis regardless of molt cycle. HSP70s have been implicated in numerous roles within the YO: provide support for increased protein synthesis and glandular growth during premolt (Rybczynski and Gilbert, 1995b); targeting of proteins to lysosomes (Stuart et al., 1995), cholesterol uptake (Brown and Golstein, 1986), and phosphoprotein phosphatase activation of ribosomal protein 6 (S6K) which ultimately initiates ecdysteroidogenesis (Song and Gilbert, 1995; Abuhagr et al., 2014).

\section{Oxidative Stress}

Reactive oxygen species (ROS) include superoxide anions $\left(\mathrm{O}_{2}{ }^{-}\right)$and hydrogen peroxide $\left(\mathrm{H}_{2} \mathrm{O}_{2}\right)$, and reactive nitrogen species (RNS) typically include nitric oxide (NO). ROS and RNS are respiratory byproducts that also hold an important place in cell signaling, immune and antimicrobial responses, and redox-state feedback mechanisms. Accumulation of excessive ROS 
and RNS within cells can lead to oxidative stress and initiate expression of ROS-scavengers in order to prevent lethal cellular damage. Two distinct patterns of abundance for ROS-scavenging proteins are indicative of the metabolic switch that occurs when the YO becomes activated and ecdysteroidogenesis begins (Fig. 25).

Within the YO there are several sources of ROS. As a steroidogenic gland, the YO is rich in mitochondria during all molt stages (Buchholz and Adelung, 1980). Leakage of electrons from complexes I and II within the mitochondria produce superoxide anions, which are subsequently converted to hydrogen peroxide and by superoxide dismutase (SOD). In crustaceans, the utilization of copper in hemocyanin has led to the use of manganese SOD (MnSOD), rather than the more common copper-zinc SOD (Brouwer et al., 2003). The mature crustacean cytosolic MnSOD is a $24.8 \mathrm{kDa}$ peptide and typically forms dimers (Brouwer et al., 2003). Interestingly, two isoforms of cytosolic MnSOD were identified with an increased abundance in the intermolt stage, and a third isoform increased in abundance in the early, mid, and late premolt stages (Fig. 24). Differences in the molecular weight of these isoforms suggest PTM-dependent activation during conditions of oxidative stress. It is likely that the two isoforms upregulated in the intermolt stage represent PTMmodification of the full-length $31.4 \mathrm{kDa}$ proteins before proteolytic cleavage. Activation of the YO and subsequent generation of superoxide anions during premolt would reasonably lead to proteolytic activation of the protein and subsequent dimerization (Brouwer et al., 2003).

While $\mathrm{H}_{2} \mathrm{O}_{2}$ is important in cell signaling and the immune response, it can also readily decompose into a hydroxyl radical and cause severe cellular damage, particularly to the mitochondria (Giorgio et al., 2007). Hydrogen peroxide is the most common source of oxidative stress in the crustacean YO. Even though MnSOD is necessary to partition destructive superoxide radicals, the generation of $\mathrm{H}_{2} \mathrm{O}_{2}$ must be further scavenged and reduced by catalase, glutathione 
peroxidase, or peroxiredoxin. A significant source of $\mathrm{H}_{2} \mathrm{O}_{2}$ in the $\mathrm{YO}$ originates from the Halloween genes used in ecdysteroid synthesis. The Halloween genes are a class of cytochrome P-450 monooxygenases responsible for several hydroxylations of ecdysteroid within the YO during the early steps of ecdysteroidogenesis (Mykles, 2011). Prenylcysteine oxidase, a previously discussed homeostatic protein, is an additional source of $\mathrm{H}_{2} \mathrm{O}_{2}$ in the $\mathrm{YO}$.

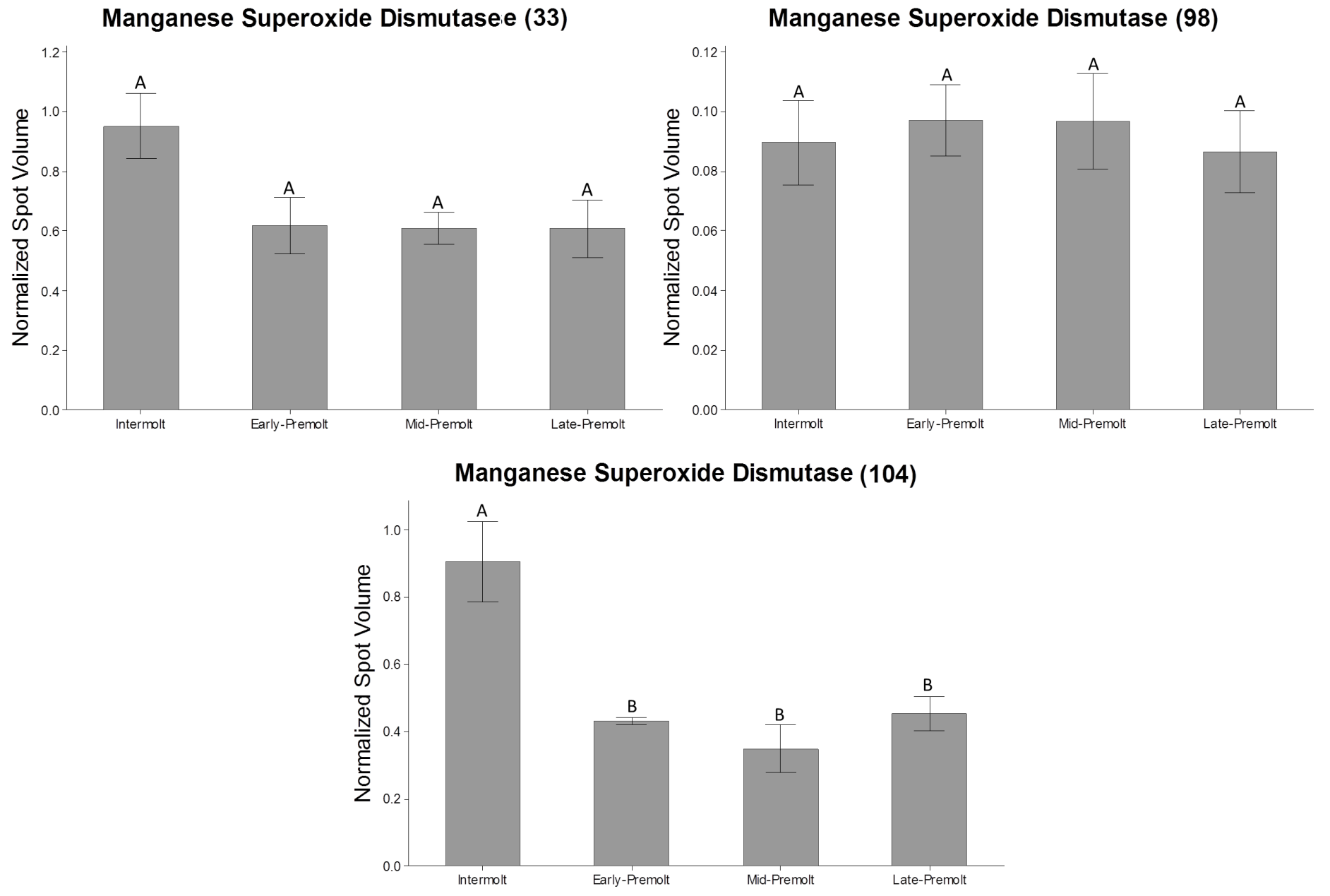

Figure 24. Levels of manganese superoxide dismutase proteins in the YO of G. lateralis over the course of the molt cycle. Spot volumes were obtained by normalizing against the volume of all proteins and show means \pm s.e.m. $(\mathrm{N}=4)$. Molt stages that do not share a letter within individual graphs are significantly different (Tukey's HSD). Spot numbers are shown in parentheses. 
Scavenging of hydrogen peroxide occurs through the enzymes catalase, peroxiredoxin, and glutathione peroxidase. Catalase is a ubiquitous cytosolic enzyme that rapidly converts hydrogen peroxide into oxygen and water molecules and is arguably one of the most important intracellular scavenger of hydrogen peroxide (Chelikani et al., 2004). The identification of seven isoforms of catalase in all molt stages of the YO is a testament to the importance of this enzyme. Analysis of the mud crab, Scylla serrata, showed escalated rates of catalase activity in the premolt and late postmolt animals, indicative of the increased metabolic activity and thus ROS synthesis during these stages (Salaenoi et al., 2014). Observed differences in molecular weight again indicate PTMs that may be utilized during times of reduced oxidative stress, or a stimulated immune response, to suppress excessive hydrogen peroxide scavenging.

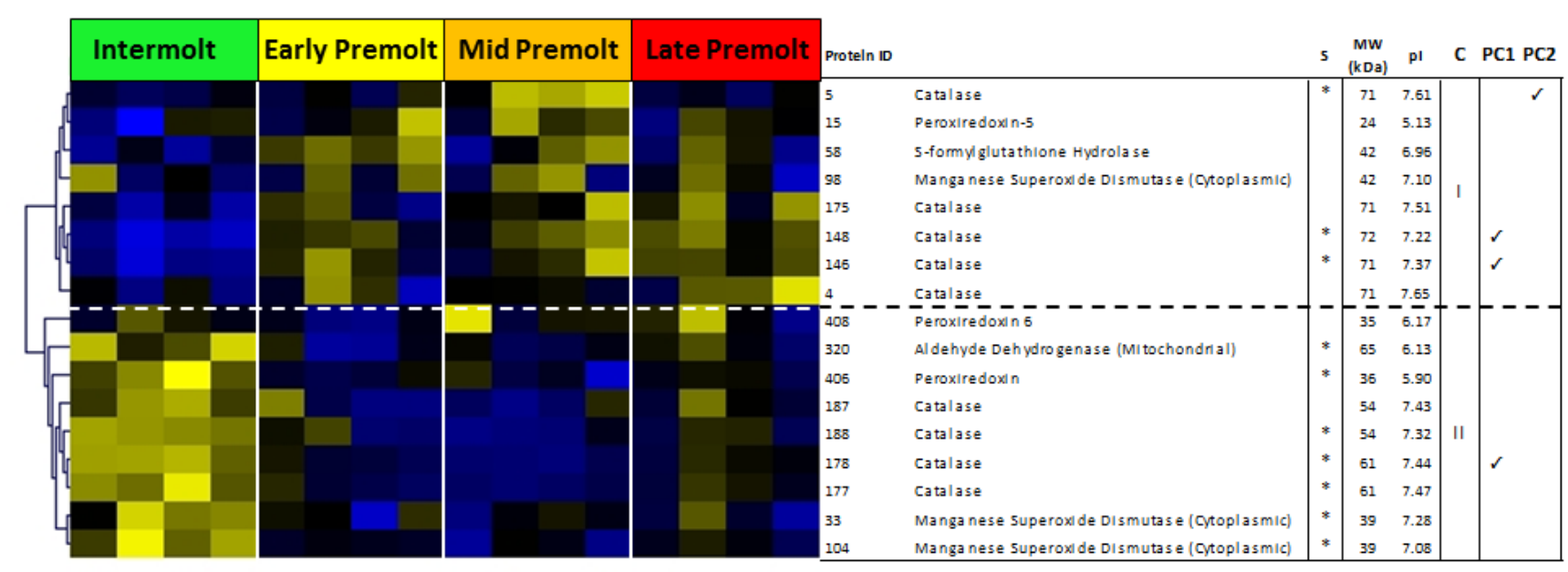

Abundance change:

$-1.98$

$-0.16$

2.68

Figure 25. Hierarchal clustering using Pearson's correlation depicting relative protein abundance (normalized spot volume) for all detected oxidative stress proteins within the Y-organ of Gecarcinus lateralis. Check marks associated with individual protein spots indicate proteins which changed significantly in abundance across the molt stages and exhibit a strong contribution towards the separation among molt stages displayed along the respective component axis (see Fig. 13B and Table 4). 
Corresponding with the changes in catalase activity, increased cellular glutathione (GSH) was observed in premolt mud crabs (Salaenoi et al., 2014). GSH is a non-enzymatic antioxidant utilized by glutathione peroxidase to reduce peroxides (Halliwell and Guttering, 2007). Sformylglutathione hydrolase catalyzes the synthesis of cellular GSH. Increased abundance of this GSH-synthesizing enzyme during the premolt stages is indicative of an increased need for an antioxidant response. GSH itself can also mediate NO transport and storage (Foyer and Noctor, 2005). As previously described, NO is a vital signaling molecule in the "summation" phase of MIH regulation of ecdysteroidogenesis in the YO (Chang and Mykles, 2011).

Peroxiredoxins (Prxs) are another class of highly abundant peroxide-scavenging enzymes. Prxs vary by sub-cellular location, with Prx5 typically found in the peroxisomes and mitochondria, and Prx6 in the cytoplasm (Cox et al., 2010). The variation in observed Prx isoforms in intermolt versus premolt may be indicative of different sources of hydrogen peroxide during the molt stages. Increased abundance of Prx 5 in premolt can be explained by increased mitochondrial activity, thus $\mathrm{H}_{2} \mathrm{O}_{2}$, associated with elevated energy demand to supply ecdysteroid synthesis. Additionally, peroxisomal degradation of long-chain fatty acids during premolt would contribute to the energetic demand of ecdysteroidogenic cells, as well as provide a substrate for synthesis of new cellular material for the hypertrophying cells. Prx6 during intermolt may play a role in regulation of oxidative species associated with MIH signaling.

Finally, oxidative stress in the form of $\mathrm{H}_{2} \mathrm{O}_{2}$, has recently been shown to induce synthesis and activity of the multifunctional transglutaminase protein. Increased $\mathrm{H}_{2} \mathrm{O}_{2}$ induced TGF $\beta$ mediated synthesis of transglutaminase, which in turn catalyzed the formation of protein aggregates within the cell. Protein aggregation has previously been thought of as a passive grouping of oxidatively damaged proteins, however this evidence suggest that TGase may be 
involved in an active response to oxidative stress (Shin et al., 2008). Increased abundance of intracellular TGase in the premolt YO may be indicative of an oxidative stress response independent of the commonly known antioxidant molecules previously discussed. 


\section{CONCLUDING REMARKS}

In this study, changes in the physiology of the Y-organ appear to be driven by molt-stage specific protein expression which supports the cellular growth and energetic demands of ecdysteroidogenesis. Visual representation of the changes in protein abundance within functional categories is provided in Figure 26. Activation of the $\mathrm{YO}$ and initiation of ecdysteroidogenesis are initially visible through changes in cytoskeletal elements and structural rearrangement of the YO. Abundance of tubulins in the premolt stages is indicative of the cytoskeletal rearrangements that would support trafficking of cholesterol to the mitochondria and ER for ecdysteroid synthesis. These cytoskeletal changes are also indicative of vesicular secretion of ecdysteroids, a mechanism which has been previously proposed but have yet to be fully accepted. Similar patterns of actin and tubulin expression in the intermolt and late premolt $\mathrm{YO}$ are representative of degeneration of the $\mathrm{YO}$ and return to the basal state, a pattern that is repeated in several other functional categories.

Activation of ecdysteroidogenesis in the early premolt YO consists of well-defined changes in protein expression from that of the intermolt YO. Increases in proteins involved with energy metabolism are representative of the increased energy demand to sustain protein synthesis and cell growth as the YO becomes activated. Identification of several proteins related to the metabolic intermediate $\alpha-\mathrm{KG}$ support proposed mTOR mediated activation of ecdysteroidogenesis. Further, proteomic analysis of the early premolt $\mathrm{YO}$ has indicated an increased immune response, suggesting a physiological preparation for the vulnerability that comes with ecdysis. 


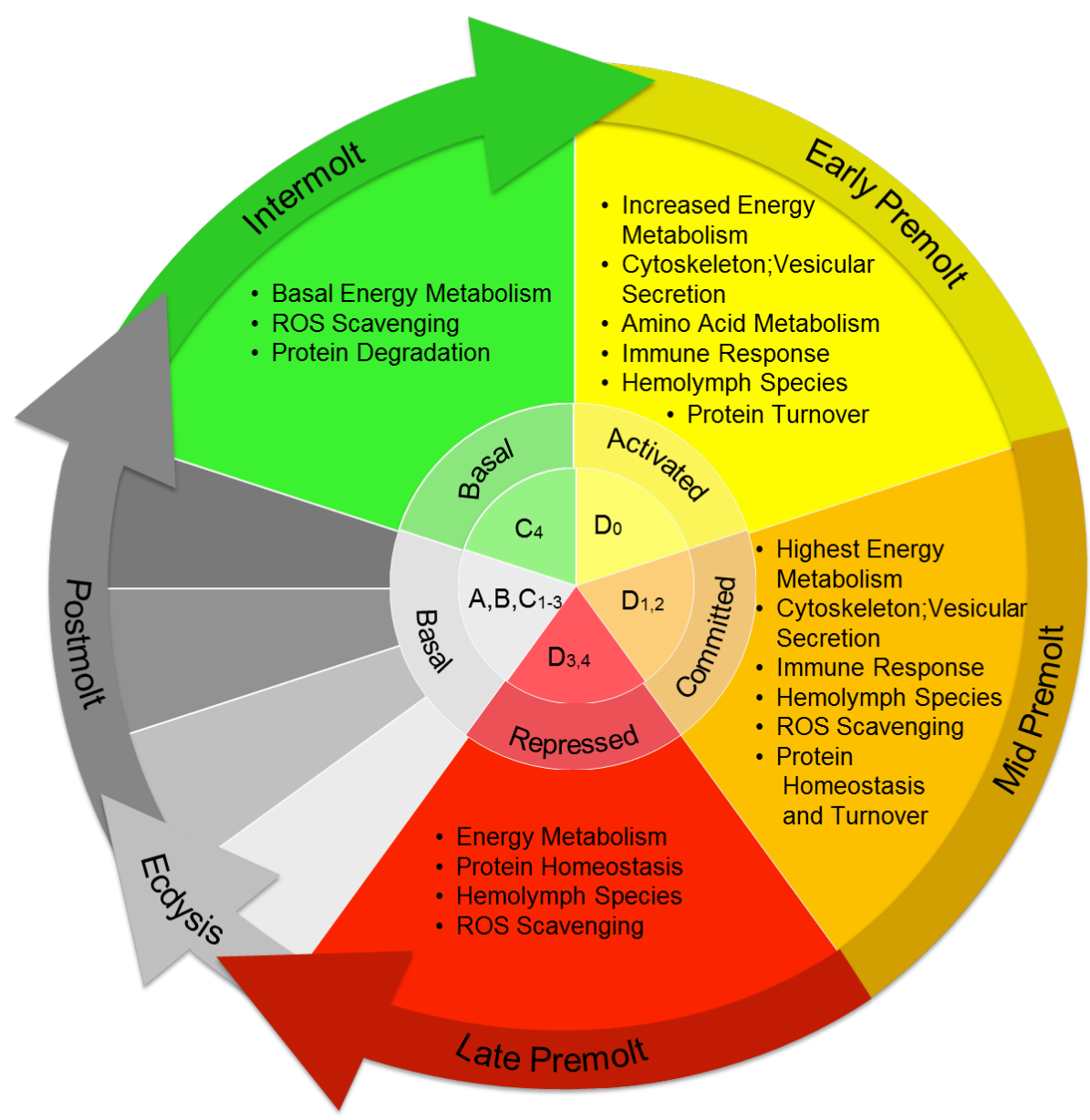

Figure 26. Classification of major functional categories contributing to each molt stage in the Yorgan of Gecarcinus lateralis. Inner circles represent the molt stage abbreviation and activation state of the Y-organ. Proteomic analysis of ecdysal and postmolt crabs have yet to be conducted.

The changes in protein abundance that distinguish the intermolt stage from early premolt were carried over, and amplified, in the mid premolt YO. The transition from early premolt to mid premolt is of particular interest as the animal is now 'committed' to molting and is refractory to manipulations that would suspend molting in an early premolt animal, such as limb bud autotomy or exogenous MIH. Principle component analysis of the G. lateralis proteome provides evidence that this commitment to molting may be regulated on a proteomic level within the YO. Specifically, the proteins which most significantly contributed to this distinct mid premolt stage were estradiol 17- $\beta$ dehydrogenase, Rab-GDI, cathepsin D, catalase, ATP synthase, and transglutaminase. Though diverse in function, these data suggest that the transition to the mid- 
premolt YO involves several levels of regulation, thus contributing to the finality of such a transition. The significant contributions of both estradiol 17- $\beta$ dehydrogenase and transglutaminase to the mid premolt stage signify a need for further understanding of their physiological role within the YO.

Patterns of protein abundance within the late premolt YO revealed an interesting mosaic of intermolt-like and premolt-like protein expression. High ecdysteroid titers during late premolt repress further ecdysteroidogenesis. Trends in protein expression were observed to be highly correlated between the early, mid, and late premolt stages, and distinct from intermolt. Due to the not yet repressed state of the late premolt samples in this study, these correlative patterns of expression are indicative of the ecdysteroidogenic state of the YO at this point. Future analysis of a repressed late premolt $\mathrm{YO}$ would help to elucidate the cellular mechanisms that cause the $\mathrm{YO}$ to become repressed just before ecdysis. Interestingly, expression of several cytoskeleton, immune response, and oxidative stress proteins which were absent in early and mid premolt were highly expressed in intermolt and moderately expressed in late premolt. The complexity of the late premolt YO suggests maintenance of ecdysteroidogenic capacity alongside preparation for degeneration to the basal state.

Future studies on the molting gland of G. lateralis should include postmolt YOs in the repressed state. The data presented in this study suggest a distinct 'on' switch characterized by pronounced changes in expression of energy metabolism, immune response, proteostasis, and oxidative stress proteins from intermolt to early premolt. Characterization of the postmolt YO would reveal whether a similar 'off' switch in protein expression occurs immediately after ecdysis, or whether return to the basal state is a slow progression for which preparation begins in the late postmolt state. 
Although not endogenous to the YO, patterns of expression of hemocyanin and cryptocyanin proteins provide supporting evidence for their molt-cycle related roles in crustaceans. Changes in molecular weight of hemocyanin correspond to previously identified cleaved peptides with antimicrobial function. This, alongside the observed changes in immune related proteins may suggest a molt stage specific immune-related role of the YO.

The findings of this study provide valuable insight into the physiological changes that regulate and are associated with progression through the molt cycle in the YO. Identification of expression patterns of these proteins are just the first step. Future investigations including rates of enzyme activity, post translational modifications, and tissue cultures will help to elucidate the function and importance of these specific proteins. 


\section{REFERENCES}

2-D Quant Kit (80-6483-56) Product Information, GE Healthcare Bio-Sciences).

Abuhagr, A. M., MacLea, K. S., Chang, E. S., and Mykles, D. L. (2014). Mechanistic target of rapamycin (mTOR) signaling genes in decapod crustaceans: cloning and tissue expression of mTOR, Akt, Rheb, and p70 S6 kinase in the green crab, Carcinus maenas, and blackback land crab, Gecarcinus lateralis. Comparative Biochemistry and Physiology, Part A, 168, 25-39.

Abuhagr, A. M., MacLea, K. S., Mudron, M. R., Chang, S. A., Chang, E. S., and Mykles, D. L. (2016). Roles of mechanistic target of rapamycin and transforming growth factor- $\beta$ signaling in the molting gland (Y-organ) of the blackback lan crab, Gecarcinus lateralis, 198, 15-21.

Anderson, K. (2010). Globalization's effects on word agricultural trade, 1960-2050. Philosophical Transactions of the Royal Society B, 365, 3007-3021.

Babu, B. T., Shyamasundari, K., and Rao, K.H. (1989). Cytoarchitecture of ecdysal gland/Y-organ of Charybdis annulata (Fabricius) (Crustacea: Decapoda: Brachyura). Raffles Bulliten of Zoology, 16, 309-354.

Bateman, K. S., Hicks, R. J., and Stentiford, G. D. (2011). Disease profiles differ between populations of pre-recruit and recruit edible crabs (Cancer pagurus) from a major commercial fishery. International Council for the Exploration of the Sea. Journal of Marine Science, 68, 2044-2052.

Berth, M., Moser, F. M., Kolbe, M., and Bernhardt, J. (2007). The state of the art in the analysis of two-dimensional gel electrophoresis images. Applied Microbiology and Biotechnology, 76, 1223-1243.

Birkenbiel, H. (1983). Ultrastructural and immunocytochemical investigation of ecdysteroid secretion by the prothoracic gland of the warmth Galleria mellonella. Cell and Tissue Research, 299, 433-441.

Bliss, D. E. (1968). Transition from water to land in decapod crustaceans. American Zoologist, 8, 355-392.

Bliss, D. E. (1979). From sea to tree: Saga of a land crab. American Zoologist, 19 (2), 385-410.

Bliss, D. E., and Boyer, J. R. (1964). Environmental regulation of growth in the decapod crustacean Gecarcinus lateralis. General and Comparative Biology, 4 (1), 15-41.

Bliss, D. E., Van Montfrans, J., Van Montfrans, M., Boyer, J. R. (1978). Behavior and growth of the land crab Gecarcinus lateralis (Freminville) in southern Florida. Bulletin of the American Museum of Natural History, 160 (2), 113-151. 
Bradford, M. M. (1976). A rapid and sensitive method for the quantification of microgram quantities of protein utilizing the principle of protein-dye binding. Analytical Biochemistry, $72,248-254$.

Bright, D. B., and Hogue, C. L. (1972). A synopsis of the burrowing land crabs of the world and list of their arthropod symbionts and burrow associates. Los Angeles County National History Museum, Contributions in Science, 220.

Brouwer, M., Hoexum Brouwer, T., Grater, W., and Brown-Peterson, N. (2003). Replacement of a cytosolic cooper/zinc superoxide dismutase by a novel cytosolic manganese superoxide dismutase in crustaceans that use copper (haemocyanin) for oxygen transport. Journal of Biochemistry, 374, 219-228.

Brown, M. S. and Goldstein, J. L. (1986). A receptor-mediated pathway for cholesterol homeostasis. Science, 232 (4746), 34-47.

Bruce, M. J., and Chang, E. S. (1984). Demostration of a molt-inhibiting hormone from the sinus gland of the lobster, Homarus americanus. The Journal of Comparative Biochemistry and Physiology A, 79, 421-424.

Buchholz, C. and Adelung, D. (1980). The ultrastructural basis of steroid production in the Yorgan and the mandibular organ of the crabs Hemigrapsus nudus (Dana) and Carcninus maenas L. Cell and Tissue Research, 206, 83-94.

Capistrán-Barradas, A., Defeo, O., and Moreno-Casasola, P. (2003). Density and population structure of the red land crab Gecarcinus lateralis in a tropical semi-deciduous forest in Veracruz, Mexico. Interciencia, 28 (6), 323-327.

Cerenius, L., Jiravanichpaisal, P., Liu, H. P., and Söderäll, I. (2010). Invertebrate Immunity: Chapter 13, Crustacean Immunity. Autstin, TX, Landes Bioscience and Springer Science and Business Media.

Chandel, N. S. (2015). Navigating Metabolism. Cold Spring Harbor Laboratory Press. Cold Spring Harbor, New York, USA

Chang, E. S. (1995). Physiological and biochemical changes during the molt cycle in decapod crsutaceans: an overview. Journal of Experimental Marine Biology and Ecology, 193, 1-14.

Chang, E. S. and Mykles, D. L. (2011). Regulation of crustacean molting: a review and our perspectives. General and Comparative Endocrinology, 172, 323-330.

Chang, E. S., Sage, B. A., and O'Connor, J. D. (1976). The qualitative and quantitative determinations of ecdysones in tissues of the crab, Pachygrapsus crassipes, following molt induction. General and Comparative Endocrinology, 30, 21-33. 
Chassard-Bouchaud, C., and Hubert, M. (1975) Sur l'existence de vesicules de reticulum endoplasmique lisse dans l'organe Y de Carcinus maenas. Comptes Rendus de l'Academie des Sciences, 281, 707-709.

Chelikani, P., Fita, I., and Loewen, P. C. (2004). Diversity of structures and properties among catalases. Cellular and Molecular Life Sciences, 61 (2), 192-208.

Cheng, J. H., and Chang, E.S. (1991). Ecdysteroid treatment delays ecdysis in the lobster, Homarus americanus. Biological Bulletin, 181, 169-174.

Cheng, W., and Chen, J. C. (2002). Effects of environmental factors on the immune responses of freshwater prawn Macrobrachium rosenbergii and other decapod crustaceans. Journal of Taiwan Fisheries Research, 29 (1), 1-19.

Chung, J. S. (2008). A trehalose 6-phosphate synthase gene of the hemocytes of the blue crab, Callinectes sapidus: cloning, the expression, its enzyme activity and relationship to hemolymph trehalose levels. Saline Systems, 4, 18.

Chung, J. S., and Webster, S. G. (2003). Moult cycle-related changes in biological activity of moult-inhibiting hormone $(\mathrm{MIH})$ and crustacean hyperglycemic hormone $(\mathrm{CHH})$ in the crab, Carcinus maenas. European Journal of Biochemistry, 270, 3280-3288.

Chung, J. S., and Webster, S. G. (2005). Dynamics of in vivo release of molt-inhibiting hormone and crustacean hyperglycemic hormone in the shore crab, Carcinus maenas. Endocrinology, $146,5545-5551$.

Collins, F. S., Morgan, M., and Patrinos, A. (2003). The Human Genome Project: lessons from large-scale biology. Science, 300, 286-290.

Conus, S. and Simon, H. U. (2010). Cathepsins: key modulators of cell death and inflammatory responses. Biochemical Pharmacology, 76, 1374-1382.

Cooper, A. J. L., Rex Sheu, K. F., Burke, J. R., Onodera, O., Strittmatter, W. J., Roses, A. D., and Blass, J. P. (1997). Transglutaminase-catalyzed inactivation of glyceraldehyde 3-phosphate dehydrogenase and $\alpha$-ketoglutarate dehydrogenase complex by polyglutamine domains of pathological length. Proceedings of the National Academy of Science, 94, 12604-12609.

Covi, J. A., Bader, B. D., Chang, E. S., and Mykles, D. L. (2010). Molt cycle regulation of protein synthesis in skeletal muscle of the blackback land crab, Gecarcinus lateralis, and the differential expression of a myostatin-like factor during atrophy induced by molting or unweighting. Journal of Experimental Biology, 213, 172-183.

Covi, J. A., Chang, E. S., and Mykles, D. L. (2009). Conserved role of cyclic nucleotides in the regulation of ecdysteroidogenesis by the crustacean molting gland. Comparative Biochemistry and Physiology A, 152, 470-477.

Covi, J. A., Chang, E. S., and Mykles, D. L. (2012). Neuropeptide signaling mechanisms in crustacean and insect molting glands. Invertebrate Reproduction and Development, 56, 33-49. 
Covi, J. A., Kim, H. W., and Mykles, D. L. (2008). Expression of alternatively spliced transcripts for a myostatin-like protein in the blackback land crab, Gecaricnus lateralis. Comparative Biochemistry and Physiology A, 150, 423-430.

Cox, A. G., Winterbourn, C. C., and Hampton, M. B. (2010). Mitochondrial peroxiredoxin involvement in antioxidant defence and redox signalling. Biochemical Journal, 425, 313-325.

Cuesta, J. A., Garcia-Guerrero, M. U., and Hendrickx, M. E. (2007). The complete larval development of Johngarthia planatus (Brachyura: Grapsoidea: Gecarcinidae) described from laboratory reared material, with notes on the affinity of Gecarcinus and Johngarthia. The Journal of Crustacean Biology, 27 (2), 263-277.

Dahm, R. (2008). Discovering DNA: Friedrich Miescher and the early years of nucleic acid research. Human Genetics, 122, 565-581.

Das, S. and Mykles, D. L. (2016). A comparison of resources for the annotation of a de novo assembled transcriptome in the molting gland (Y-organ) of the blackback land crab, Gecarcinus lateralis. Integrative and Comparative Biology, 56, 1103-1112.

Das, S., Pitts, N. L., Mudron, M. R., Durica, D. S., and Mykles, D. L. (2016). Transcriptome analysis of the molting gland (Y-organ) from the blackback land crab, Gecarcinus lateralis. Comparative Biochemistry and Physiology, Part D, 17, 26-40.

Decker, H. and Jaenicke, E. (2004). Recent findings on phenoloxidase activity and antimicrobial activity of hemocyanins. Developmental and Comparative Immunology, 28, 673-687.

Destoumieux-Garzón, D., Sauliner, D., Garnier, J., Jouffrey, C., Bulet, P., and Bachere, E. (2001). Antifungal peptides are generated from the $\mathrm{c}$ terminus of shrimp hemocyanin in response to microbial challenge. Journal of Biological Chemistry, 276 (50), 47070-47077.

Drach, P. (1939). Mue et cycle d'intermue ches les Crustacés Décapodes. Annzles de l'Institut Océanographique, Monaco, 19, 103-391.

Durán, R., and Hall, M. (2012). Glutaminolysis feeds mTORC1. Cell Cycle, 11, 4107-4108.

Durstewitz, G. and Terwilliger, N. B. (1997a). cDNA cloning of a developmentally regulated hemocyanin subunit in the crustacean Cancer magister and phylogenetic analysis of the hemocyanin gene family. Molecular Biology and Evolution, 14 (3), 266-276.

Durstewitz, G and Terwilliger, N. B. (1997b). Developmental changes in hemocyanin expression in the Dungeness crab, Cancer magister. Journal of Biological Chemistry, 272 (7), 4347-4350.

Ehrhardt, J. P., and Niaussat, P. (1970). Ecologie et physiologie du brachyoure terrestre Gecarcinus planatus Stimpson (d'aprés les individus de l'atoll de Clipperton). Bulletin de la Société Zoologique de France, 95, 41-54. 
Food and Agriculture Organization (FAO) of the United Nations (2016). The state of world fisheries and aquaculture; contributing to food security and nutrition for all. Fisheries and Aquaculture Department, Rome, IT.

Foyer, C. H. and Noctor, G. (2005). Redox homeostasis and antioxidant signaling: a metabolic interface between stress perception and physiological responses. The Plant Cell, 17 (7), 18661875.

Gilbert, L. I., and Rewitz, K. F. (2009). The function oand evolution of the Halloween genes: the pathway to the arthropod molting hormone. Ecdysone: Structures and Function. Springer, Netherlands. Pp. 231-269.

Giogio, M., Trinei, M., Migliaccio, E., and Pelicci, P. G. (2007). Hydrogen peroxide: a metabolic by-product or a common mediator of ageing signals? National Review of Molecular Cell Biology, 8, 722-728.

Görg, A., Weiss, W., and Dunn, M. J. (2004). Current two-dimensional electrophoresis technology for proteomics. Proteomics, 4, 3665-3685.

Görg, A., Drews, O., Luck, C., Weiland, F., and Weiss, W. (2009). 2-DE with IPGS. Electrophoresis, 30, S122-132.

Green, P. T. (1997). Red crabs in rain forest in Christmas Island, Indian Ocean: activity, patterns, density and biomass. The Journal of tropical Ecology, 13, 17-38.

Green, P. T. (2004). Field observations of moulting and moult increment in the red land crab, Gecarcoidea natalis (Brachyura, Gecarcinidae), on Christmas Island (Indian Ocean). Crustaceana, 77 (1), 125-128.

Hall, P.F., Charpponnier, C., Nakamura, M., and Gabbiani, G. (1979). The role of microfilaments in the response of adrenal tumor cells to adrenocorticotropic hormone. Journal of Biological Chemistry, 254, 9080-9084.

Halliwell, B., and Gutteridge, J. M. C. (2007). Free radicals in biology and medicine, 4th ed. Oxford Universiy Press. New York, NY, USA.

Hamer, M. S. (2015). The proteomic response of the Carcinus maenas Y-organ over the course of the molt cycle (Unpublished Master's Thesis). California Polytechnic State University, San Luis Obispo, CA, USA.

Hanström, B. (1939). Hormones in invertebrates. Oxford University Press, London, UK.

Hartnoll, R. G. (1988). Evolution, systematics, and geographical distribution. Biology of Land Crabs. Cambridge University Press. New York, NY, USA. Pp. 20-54. 
Herreid, F. C. II, Full, R. J. (1988) Energetics and locomotion. Biology of Land Crabs. Cambridge University Press. New York, NY, USA. Pp. 333-377.

Holland, C. A., and Skinner, D. M. (1976). Interactions between molting and regeneration in the land crab. Biological Bulletin, 150, 222-240.

Hopkins, P. M. (1982). Effects of neurosecretory factors on in vivo levels of ecdysteroids in hemolymph of the fiddler crab, Uca pugilator. American Zoologist, 22, 938.

Hopkins, P. M. (2009). Crustacean ecdysteroids and their receptors. Ecdysone: Structures and Function. Springer, Netherlands. Pp. 73-97.

Huang, K. and Fingar, D. C. (2014). Growing knowledge of the mTOR signaling network. Seminars in Cell and Developmental Biology, 36, 79-90.

Inoki, K., Li, Y., Xu, T. and Guan, K.L. (2003). Rheb GTPase is a direct target of TSC2 GAP activity and regulates mTOR signaling. Genes and Development, 17, 1829-1834.

Intra, J., Pavesi, G., and Horner, D. S. (2008). Phylogenetic analyses suggest multiple changes of substrate specificity within the Glycosyl hydrolase 20 family. BMC Evolutionary Biology, 8, 214.

Keller, R., and O'Connor, J. D. (1982). Neuroendocrine regulation of ecdysteroid production in the crab Pachygrapsus crassipes. General and Comparative Endocrinology, 46, 384.

Kellman, M., and Delfosse, B. (1993). Effect of the red land crab, Gecarcinus lateralis on leaf litter in a tropical dry forest in Veracruz, Mexico. Journal of Tropical Ecology, 9, 55-65.

Kellman, M., and Roulet, N. (1990). Nutrient flux and retention in a tropical sand-dune succession. Journal of Ecology, 78, 664-676.

Kim, H. W., Lee, S. G., and Mykles, D. L. (2005). Ecdysteroid-responsive genes, RXR and E75, in the tropical land crab, Gecarcinus lateralis: differential tissue expression of multiple RXR isoforms generates at three alternative splicing sites in the hinge and lingand-binding domains. Molecular and Cellular Endocrinology, 242, 80-95.

Klaassen, F. (1975). Ökologische und ethologische Untersuchungen zur Fortpflanzungsbiologie von Gecarcinus lateralis (Decapoda, Brachyura). Forma et Functio, 8, 101-174.

Kone, B. C. (2001). Molecular biology of natriuretic peptides and nitric oxide synthases. Cardiovascular Research, 51, 429-441.

Kuballa, A. V. and Elizur, A. (2008). Differential expression profiling of components associated with exoskeletal hardening in crustaceans. BMC Genomics, 9, 575. 
Lachaise, F., Le Roux, A., Hubert, M. and Lafont, R. (1993). The molting gland of crustaceans: localization, activity, and endocrine control (a review). Journal of Crustacean Biology, 13 (2), 198-234.

Lai, T. S., Lin, C. J., and Greenberg, C. S. (2017). Role of tissue transglutaminase-2 (TG2)mediated aminylation in biological processes. Amino Acids, 49, 501-515.

Lee, S. G., Bader, B. D., Chang, E. S. and Mykles, D. L. (2007). Effects of elevated ecdysteroid on tissue expression of three guanylyl cyclases in the tropical land crab Gecarcinus lateralis: possible roles of neuropeptide signaling in the molting gland. Journal of Experimental Biology, 210, 3245-3254.

Lee, S. Y., Lee, B. L., and Söderäll, K. (2003). Processing of an antibacterial peptide from hemocyanin of the freshwater crayfish Pacifatacus leniusculus. Journal of Biological Chemistry, 278 (10), 7927-7933.

Lee, S. G. and Mykles, D. L. (2006). Proteomics and signal transduction in the crustacean molting gland. Integrative and Comparative Biology, 46 (6), 965-977.

Liang, Z., Sottrup-Jensen, L., Aspan, A., Hall, M., and Söderhäll, K. (1997). Pacifastin, a novel $155-\mathrm{kDa}$ heterodimeric proteinase inhibitor containing a unique transferrin chain. Proceedings of the National Academy of Science, 94, 6682-6687.

Lin, X., Söderhäll, K., and Söderhäll, I. (2008). Transglutaminase activity in the hematopoietic tissue of a crustacean, Pacifasticus leniusculus, importance in hemocyte homeostasis. BMC Immunology, 9, 58.

López, J. L. (2007). Two-dimensional electrophoresis in proteome expression analysis. Journal of Chromatography, B. Analytical Technologies in the Biomedical and Life Sciences, 849, 190202.

Louda, M. S., and Zedler, P. H. (1985). Predation in insular plant dynamics: an experimental assessment of postdispersal fruit and seed survival, Enewetak Atoll, Marshall Islands. American Journal of Botan, 72, 438-445.

Lovríc, J. (2011). Introducing Proteomics. Wiley-Blackwell. West Sussex, UK.

Lu, J. Y., and Hofmann, S. L. (2006). Lysosomal metabolism of lipid-modified proteins. Journal of Lipid Research, 47, 1352-1357.

Magdeldin, S., Enany, S., Yoshida, Y., Zhang, Y., Zureena, Z., Lokamani, L., Yaoita, E., Yanamoto, T. (2014). Basics and recent advances of two-dimensional polyacrylamide gel electrophoresis. Clinical Proteomics, 11, 16.

Mason, C. A. (1970). Function of the pericardial sacs during the molt cycle in the land crab Gecarcinus lateralis. The Journal of Experimental Zoology, 174, 381-390. 
Matsuda, Y., Koshiba, T., Osaki, T., Suyama, H., Arisaka, F., Toh, Y., and Kawabata, S. (2007). An arthropod cuticular chitin-binding protein endows injured sites with transglutaminasedependent mesh. Journal of Biological Chemistry, 282, 37316-37314.

McDonald, A. A., Chang, E. S., and Mykles, D. L. (2011). Cloning of a nitric oxide synthase from green shore crab Caricnus maenas: a comparative study of the effects of eyestalk ablation on expression in the molting glands (Y-organs) or C. maenas, and blackback land crab, Gecarcinus lateralis. Comparative Biochemistry and Physiology A, 158, 150-162.

Morris, S. M. Jr. (2002). Regulation of enzymes of the urea cycle and arginine metabolism. Annual Review of Nutrition, 22, 87-105.

Muller, S., Dennemarker, J., and Reinheckel, T. (2012). Specific functions of lysosomal proteases in endocytic and autophagic pathways. Biochemical et Biophysica Acta, 1824, 34-43.

Mykles, D. L. (1997). Crustacean muscle plasticity: molecular mechanisms determining mass and contractile properties. Comparative Biochemistry and Physiology B, 117, 367-378.

Mykles, D. L. (2001). Interactions Between Limb Regeneration and Molting in Decapod Crustaceans. American Zoologist, 41, 399-406.

Mykles, D. L. (2011). Ecdysteroid metabolism in crustaceans. Journal of Steroid Biochemistry and Molecular Biology, 127, 196-203.

Mykles, D. L. (1980). The mechanism of fluid absorption at ecdysis in the American lobster, Homarus americanus. Journal of Experimental Biology, 84, 89-101.

Nakatsuji, T., Lee, C. Y., and Watson, R. D. (2009). Crustacean molt-inhibiting hormone: structure, function, and cellular mode of action. Comparative Biochemistry and Physiology A, $152,139-148$.

Nakatsuji, T., and Sonobe, H. (2004). Regulation of ecdysteroid secretion from the Y-organ by molt-inhibiting hormone in the American crayfish, Procambarus clarkii. General and Comparative Endocrinology, 135, 358-364.

Nakatsuji, T., Sonobe, H., and Watson, R. D. (2006). Molt-inhibiting hormone-mediated regulation of ecdysteroid synthesis in Y-organs of the crayfish (Procambarus clarkii): involvement of cyclic GMP and cycle nucleotide phosphodiesterase. Molecular and Cellular Endocrinology, 253, 76-82.

Nimitkul, S. (2011). Feedback Regulation of Ecdysteroid Analog on Y-Organs of the Green Crab Carcinus Maenas. Proquest, UMI Dissertation Publishing.

Ødegaard, F. (2000). How many species of arthropods? Erwin's estimate revised. Biological Journal of the Linnean Society, 71 (4), 583-597. 
Petit, V. W., Rolland, J., Blond, A., Cazevieille, C., Djediat, C., Peduzzi, J., Goulard, C., Bachere, E., Dupont, J., Destoumieuz-Garzón, D., and Rebuffat, S. (2016). A hemocyanin-derived antimicrobial peptide from the penaeid shrimp adopts an alpha-helical structure that specifically permeabilizes fungal membranes. Biochemical et Biophysica Acta, 1860, 557-568.

Prasterlux, S., Sittikankaew, K., Chumtong, P, Khamnamtong, B., and Klinbunga, S. (2011). Molecular characterization and expression of the prostaglandin reductase 1 gene and protein during ovarian development of the giant tiger shrimp, Penaeus monodon. Aquaculture, 322, 134-141.

Qiu, L. Jiang, S., Huang, J., Wang, W., Zhang, D., Wu, Q., and Yang, K. Molecular cloning and mRNA expression of cathepsin $\mathrm{C}$ gene in black tiger shrimp (Penaeus monodon). Comparative Biochemistry and Physiology, Part A, 150, 320-325.

Rbycznski, R., and Gilbert, R. I. (1995a). Prothoracicotropic hormone elicits a rapid, developmentally specific synthesis of $\beta$ tubulin in an insect endocrine gland. Developmental Biology, 169, 15-28.

Rbycznski, R., and Gilbert, R. I. (1995b). Prothoracicotropic hormone-regulated expression of a hsp 70 cognate protein in the insect prothoracic gland. Molecular and Cellular Endocrinology, $115,73-85$.

Reglero, A., and Cabezas, J. A. (1976). Glycosidases of mollusks: purification and properties of a-L-Fucosidase from Chamelea gallina L. European Journal of Biochemistry, 66, 379-387.

Ringnèr, M. (2008). What is principal component analysis? Nature Biotechnology, 26 (3), 303 304.

Robertson, A. I., and Daniels, P. A. (1989). The influence of crabs on litter processing in high intertidal mangrove forest in tropical Australia. Oecologia, 78, 191-198.

Roman, J. and Darling, J. A. (2007). Paradox lost: genetic diversity and the success of aquatic invasions. Trends in Ecology and Evolution, 22, 454-464.

Rowley, A. F., Vogan, C. L., Taylor, G. W., and Clare, A. S. (2005). Prostaglandins in non-insectan invertebrates: recent insights and unsolved problems. Journal of Experimental Biology, 208, 3-14.

Salaenoi, J., Srimeetian, P., and Mingmuang, M. (2014). Variations of catalase and glutathione activities in molting cycle of mud crab, Scylla serrata. Kasetsart Journal Natural Science, 48, 64-71.

Shaw, M. M., and Riederer, B. M. (2003). Sample preparation for two-dimensional gel electrophoresis. Proteomics, 3, 1408-1417.

Shin, D. M., Jeon, J. H., Kim, C. W., Cho. S. Y., Lee, H. J., Jang, G. Y., Jeong, E. M., Lee, D. S., Kang, J. H., Melino, G., Park, S. C., Kim, I. G. (2008). TGF $\beta$ mediates activation of 
transglutaminase 2 in response to oxidative stress that leads to protein aggregation. The FASEB Journal, 22, 2498-2507.

Shrivastava, S., and Princy, S. A. (2014). Pharmacore based approach to design inhibitors in Crustaceans: an insight into the molt inhibition response to the receptor guanylyl cyclase. Indian Journal of Experimental Biology, 52, 375-382.

Skinner, D. M. (1962). Structure and metabolism of a crustacean integumentary tissue during a molt cycle. Biological Bulletin, 123 (3), 635-647.

Skinner, D. M. (1966). Breakdown and reformation of somatic muscle during the molt cycle of the land crab, Gecarcinus lateralis. Journal of Experimental Zoology, 163, 115-124.

Skinner, D. M. (1985a). Interacting factors in the control of the crustacean molt cycle. American Zoologist, 25 (1), 275-284.

Skinner, D. M. (1985b). Molting and regeneration. The biology of Crustacea Vol. 9. Academic Press, Inc. Cambridge, MA, USA.

Skinner, D. M. and Graham, D. E. (1970). Molting in land crabs: stimulation by leg removal. Science, 169, 383-385.

Skinner, D. M. and Graham, D. E. (1972). Loss of limbs as a stimulus to ecdysis in Brachyura (true crabs). Biological Bulletin, 143, 222-233.

Skinner, D. M., Kumari, S. S. and O'Brien, J. (1992). Proteins of the crustacean exoskeleton. Integrative and Comparative Biology, 32 (3), 470-484.

Smethurst, P. A., and Griffin, M. (1996). Measurement of tissue transglutaminase activity in a permeabilized cell system: its regulation by $\mathrm{Ca}^{2+}$ and nucleotides. Journal of Biochemistry, 313, 803-808.

Smija, M. K. and Sudha Devi, A. R. (2016). Histological changes of Y-organ in Travancoriana schirnerae during molt cycle and in de-eyestalked crabs. Turkish Journal of Fisheries and Aquatic Sciences, 16, 533-544.

Smith, V. J., and Chisholm, J. R. S. (2001). Antimicrobial proteins in crustaceans. Phylogenetic perspectives on the vertebrate immune system, 484, 95-112.

Song, Q. S., and Gilbert, L. I. (1995). Multiple phosphorylation of ribosomal-protein S6 and specific protein-synthesis are required for prothoracicotropic hormone-stimulates ecdysteroid biosynthesis in the prothoracic glands of Manduca sexta. Insect Biochemistry and Molecular Biology, 25 (5), 591-602.

Spindler, K.-D., Hönl, C., Tremmel, C., Braun, S., Ruff, H. and Spindler-Barth, M. (2009). Ecdysteroid hormone action. Cell and Molecular Life Sciences, 66, 3837-3850. 
Sritunyalucksana, K. and Söderhäll, K. (2000). The proPO and clotting system in crustaceans. Aquaculture, 191, 53-69.

Stanley, D. (2006). Prostaglandind and other eicosanoids in insects: biological significance. Annual Review of Entomology, 51, 25-44.

Stentiford, G.D., D.M. Niel, E.J. Peeler, J.D. Shields, H.J. Small, T.W. Flegel, J.M. Vlak, B. Jones, F. Morado, S. Moss, J. Lotz, L. Bartholomay, D.C. Behringer, C. Hauton, and D.V. Lightner. 2012. Disease will limit future food supply from the global crustacean fishery and aquaculture sectors. Journal of Invertebrate Pathology, 110, 141-157.

Stuart, R. A., Cyr, D. M., and Neupert, W. (1994). HSP70 in mitochondrial biogenesis- from chaperoning nascent polypeptide-chains to facilitation of protein-degradation. Experientia, 50 (11-12), 1002-1011.

Strickler, R. C., and Tobias, B. (1980). Estradiol 17- $\beta$ dehydrogenase and $20 \alpha$-hydroxysteroid dehydrogenase from human placental cytosol: one enzyme with two activities? Steroids, 36 (2), 243-253.

Sugumaran, M. and Barek, H. (2016). Critical analysis of the melanogenic pathway in insects and higher animals. International Journal of Molecular Sciences, 17, 1753.

Taylor, A. C., and Davies, P. S. (1981). Respiration in the lab crab, Gecarcinus lateralis. Journal of Experimental Biology, 93, 197-208.

Taylor, J. R. A., and Kier, W. M. (2003). Switching skeletons: hydrostatic support in molting crabs. Science, 301 (5630), 209-210.

Taylor, J. R. A., and Kier, W. M. (2006). A pneumo-hydrostatic skeleton in land crabs crabs- A sophisticated dual support system enables a crab to stay mobile immediately after molting. Nature, 440, 1005.

Terman, J. R., and Kashina, A. (2013). Post-translational modification and regulation of actin. Current Opinion in Cell Biology, 25 (1), 30-38.

Terwilliger, N. B. (2012). Gene expression profile, protein production, and functions of cryptocyanin during the crustacean molt cycle. Invertebrate Reproduction and Development, $56(3), 229-235$.

Terwilliger, N. B., Dangott, L. L. and Ryan, M. M. (1999). Cryptocyanin, a crustacean molting protein: evolutionary link with arthropod hemocyanins and insect hexamerins. Proceedings of the National Academy of Science, 96, 2013-2018.

Terwilliger, N. B. and Ryan, M. C. (2006). Functional and phylogenetic analysis of phenoloxidases from Brachyuran (Cancer magister) and Brachiopod (Artemia franciscana, Triops longicaudatus) crustaceans. Biological Bulletin, 210, 38-50. 
Terwilliger, N. B., Ryan, M. C, and Towle, D. (2005). Evolution of novel functions: cryptocyanin helps build new exoskeleton in Cancer magister. Journal of Experimental Biology, 208, 24672474.

Tharia, H. A., Shrive, A. K., Mills, J. D., Arme, C., Williams, G. T., and Greenhough, T. J. (2002). Complete cDNA sequence of SAP-like pentraxin from Limulus polyphemus: implication for pentraxin evolution. Journal of Molecular Biology, 316, 583-597.

Thomas, T. M. (1974). A detailed analysis of climatological and hydrological records of South Florida with reference to man's influence upon ecosystem evolution. Environments of south Florida: Past and Present, Miami Geological Society. Miami, FL, USA. Pp 82-122.

Thongbuakaew, T., Siangcham, T., Suwansa-ard S., Elizur, A., Cummins, S. F., Sobhon, P., and Sretarugsa, P. (2016). Steroid and genes related to steroid biosynthesis in the female giant freshwater prawn, Macrobrachium rosenbergii. Steroids, 107, 149-160.

Tomanek, L. (2015). Proteomic responses to environmentally induced oxidative stress. Journal of Experimental Biology, 218, 1867-1879.

Tomanek, L. (2014). Proteomics to study adaptations in marine organisms to environmental stress. Journal of Proteomics, 105, 92-106.

Tomanek, L. and Zuzow, M. J. (2010). The proteomic response of the mussel congeners Mytilus galloprovincialis and $M$. trossulus to acute heat stress: implications for thermal tolerance limits and metabolic costs of thermal stress. Journal of Experimental Biology, 213, 35593574.

Tomiya, N., Narang, S., Park, J., Abdul-Rahman, B., Choi, O., Singh, S., Hiratake, J., Sakata, K., Betenbaugh, M. J., Palter, K. B., and Lee, Y. C. (2006). Purification, characterization, cloning of a Spodoptera frugiperda Sf9 beta-N-acetylhexosaminidase that hydrolyzes terminal Nacetylglucosamine on the N-glycan core. Journal of Biological Chemistry, 281 (28), 1954519560.

Watson, J. D. and Crick, F. H. C. (1953). Molecular structure of nucleic acids: a structure for deoxyribose nucleic acid. Nature, 171, 737-738.

Watson, R. D., Ackerman-Morris, S., Smith, W. A., Watson, C. J., and Bollenbacher, W. E. (1996). Involvement of microtubules in prothorcicotropic hormone-stimulated ecdysteroidogenesis by insect (Manduca sexta) prothoracic glands. Journal of Experimental Zoology, 276, 63-69.

Webster, S. G. (1993). High-affinity binding of putative moult-inhibiting hormone (MIH) and crustacean hyperglycemic hormone $(\mathrm{CHH})$ to membrane-bound receptors on the Y-organ of the shore crab Carcinus maenas. Proceedings of the Royal Society of London, Biology, 251, 53-59. 
Webster, S. G. (1986). Neurohormonal control of ecdysteroid biosynthesis by Carcinus maenas Y-organs in vitro and preliminary characterization of the putative molt-inhibiting hormone. General and Comparative Endocrinology, 61, 237-247.

Weist, S., Eravci, M., Broedel, O., Fuxius, S., Eravci, S., and Baumgartner, A. (2008). Results and reliability of protein quantification for two-dimensional gel electrophoresis strongly depend on the type of protein sample and the method employed. Proteomics, 8, 3389-3396.

Willems, K. A. (1982). Larval development of the land crab Gecarcinus lateralis lateralis(Freminville, 1835) (Brachyura, Gecarcinidae) reared in the laboratory. The Journal of Crustacean Biology, 2, 180-201.

Wolcott, D. L. (1991). Nitrogen excretion is enhanced during urine recycling in two species of terrestrial crab. Journal of Experimental Zoology, 259, 181-187.

Wolcott, T. G., and Wolcott, D. L. (1985). Factors influencing the limits of migratory movements in terrestrial crustaceans. Migration: mechanisms and adaptive significance. Contributions in Marine Science. Marine Science Institute. Port Aransas, TX, USA. Supplemental volume 27, 257-273.

Wolcott, T. G., and Wolcott, D. L. (1988). Availability of salts is not a limiting factor for the land crab, Gecarcinus lateralis (Freminville). Journal of Experimental Marine Biology and Ecology, 120, 199-219.

Yamanaka, N., Marques, G., and O'Connor, M.B. (2015). Vesicle-mediated steroid hormone secretion in Drosophila melanogaster. Cell, 163, 907-919.

Yokomizo, T., Ogawa, Y., Uozumi, N., Kume, K., Izumi, T., and Shimizu, T. (1996). cDNA cloning, expression, and mutagenesis study of leukotriene B4 12-hydroxydehydrogenase. Journal of Biological Chemistry. 271, 2844-2850.

Yu, X. L., Chang, E. S., and Mykles, D. L. (2002). Characterization of limb autotomy factorproecdysis $\left(\mathrm{LAF}_{\mathrm{pro}}\right)$, isolated from limb regenerates, that suspends molting in the land crab Gecarcinus lateralis. Biological Bulletin, 202, 204-212.

Zhang, Z. Q. (2011). Animal Biodiversity: an outline of higher-level classification and survey of taxonomic richness - Phylum Arthropoda, von Siebold 1848. Zootaxa, 4138, 99-103. 


\section{Supplemental Information}

APPENDIX

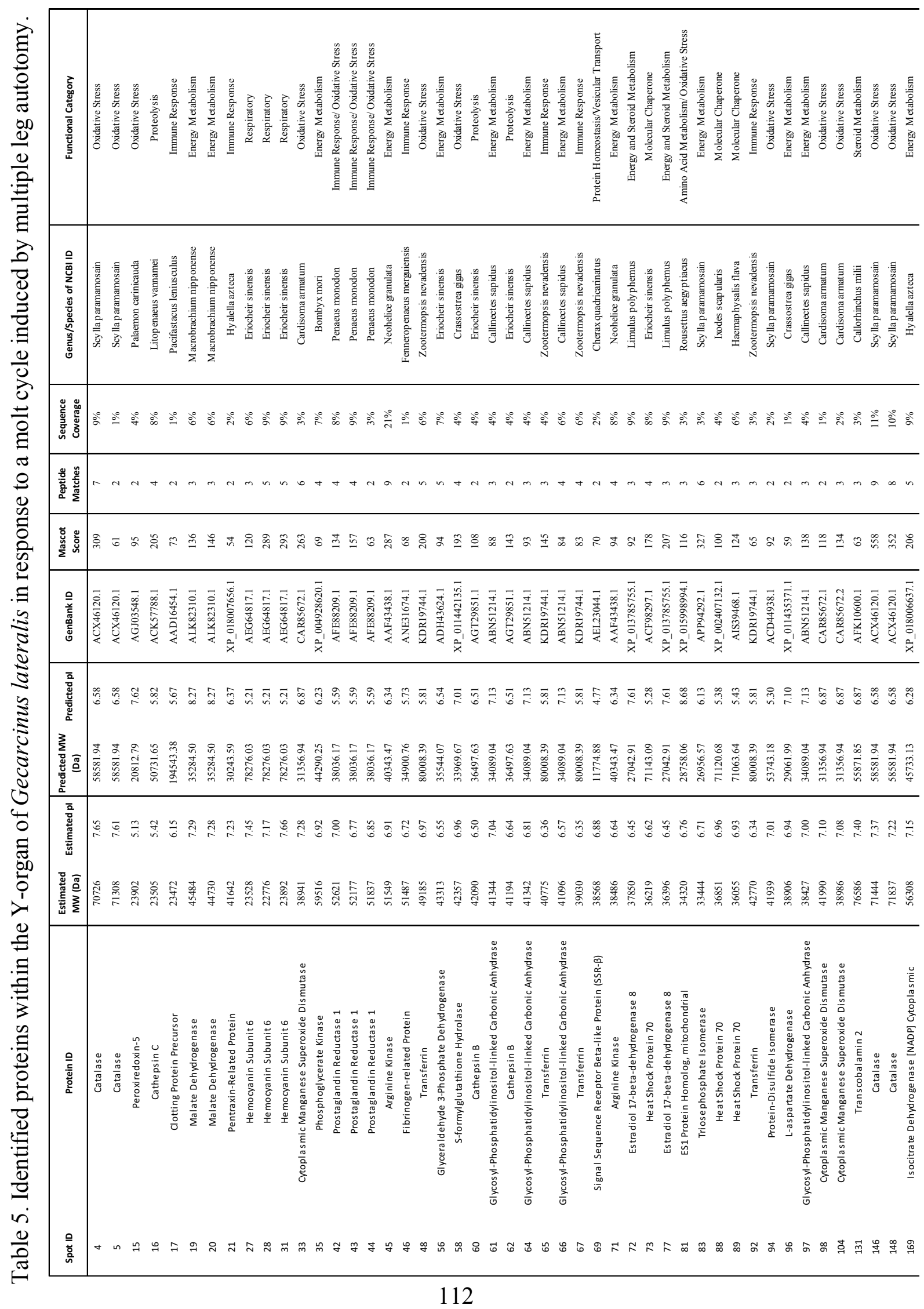




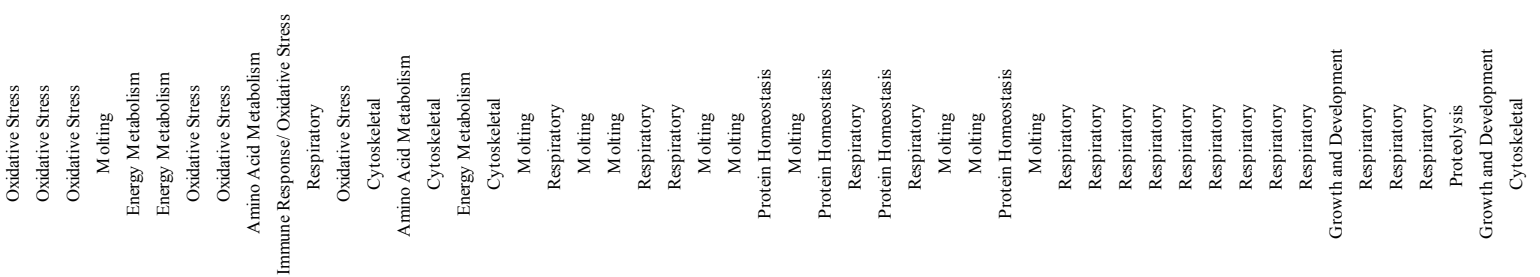

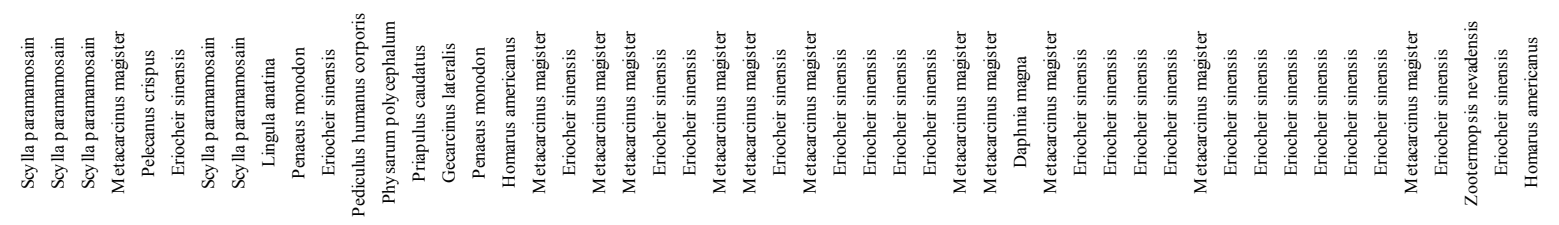

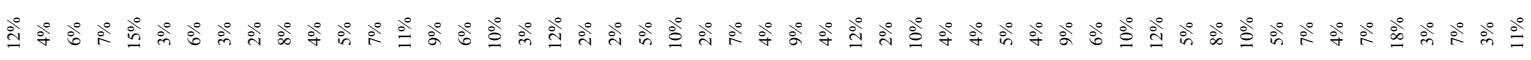

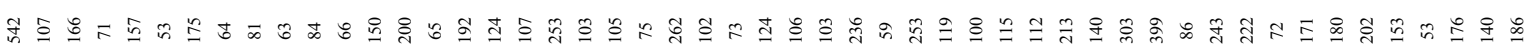

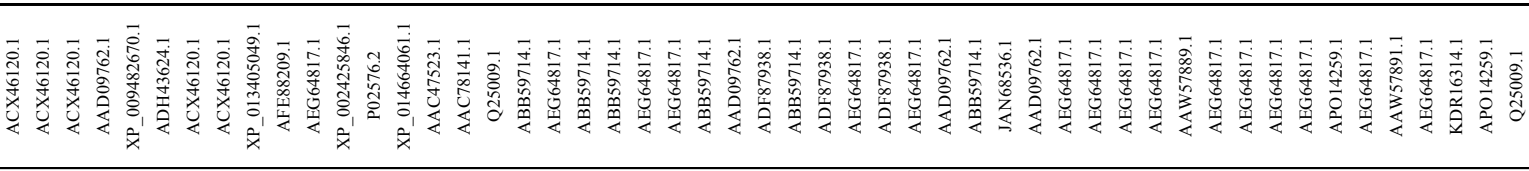

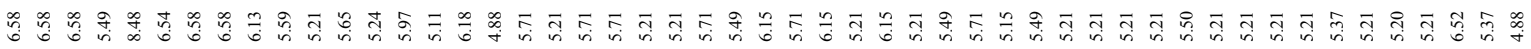

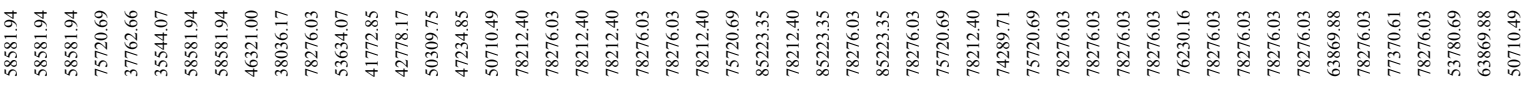

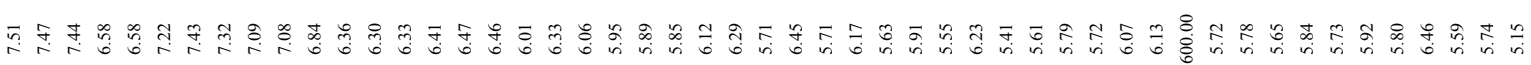

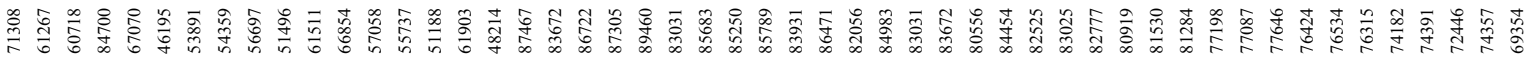

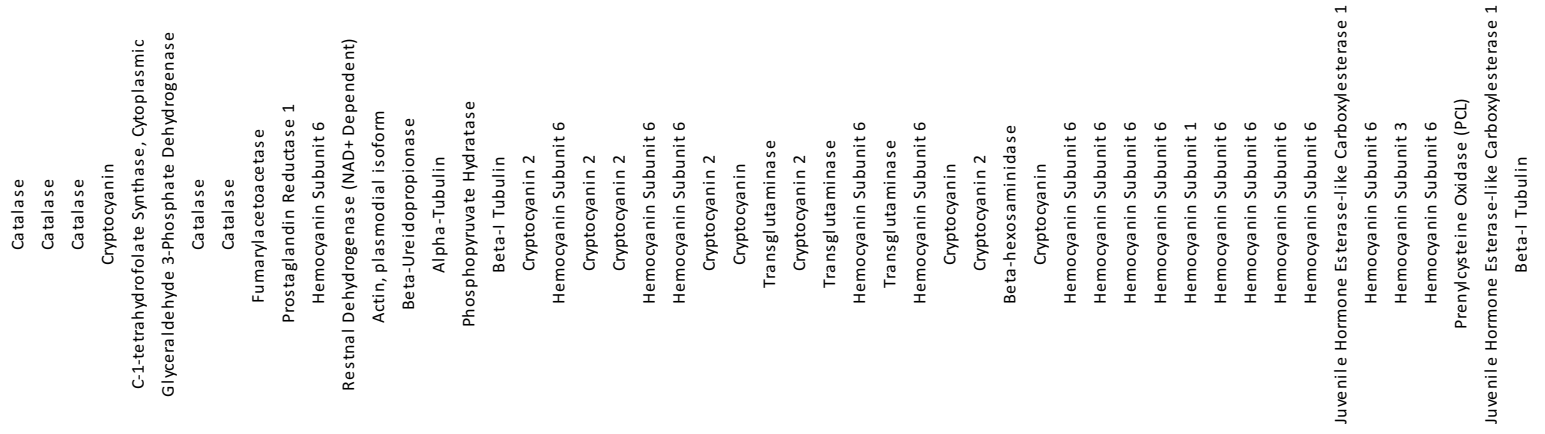

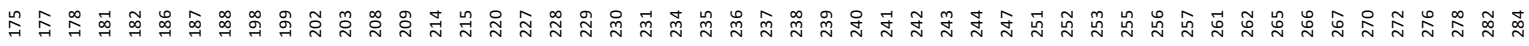




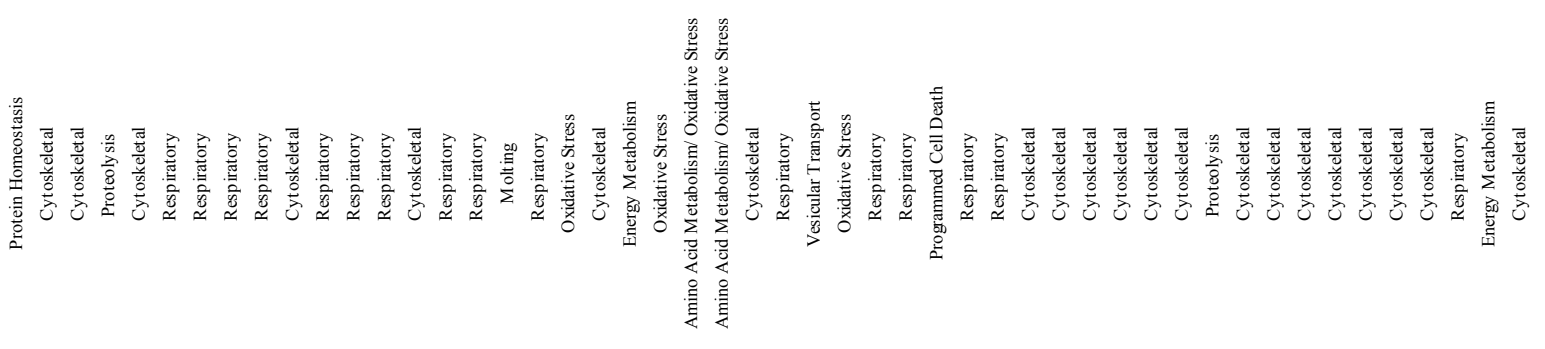

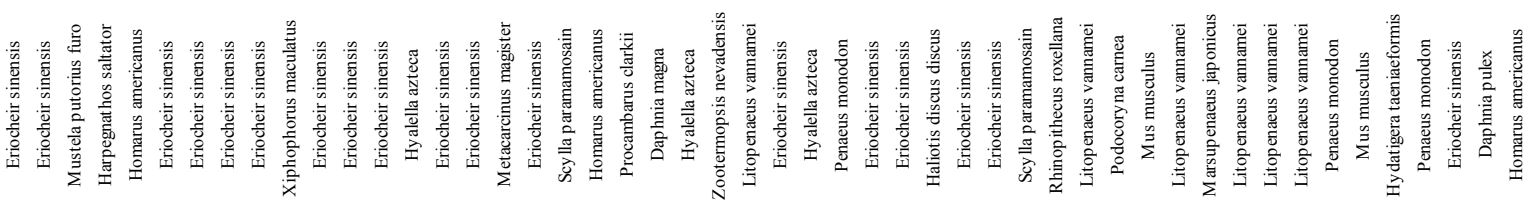

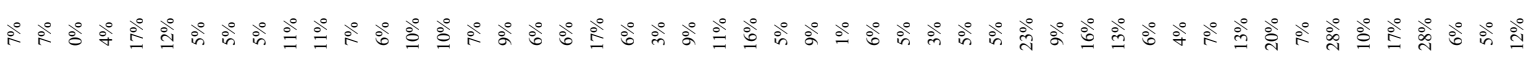

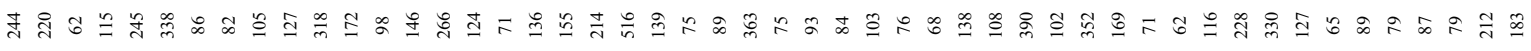

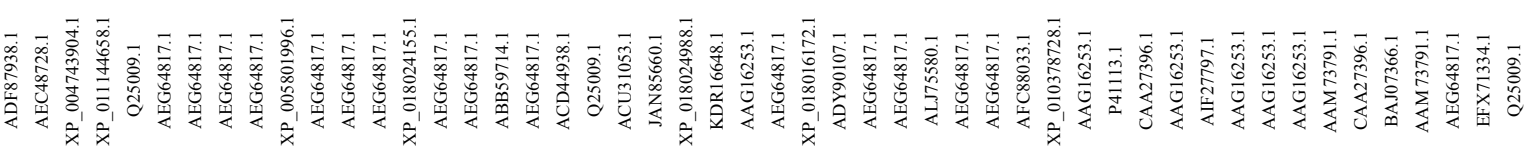

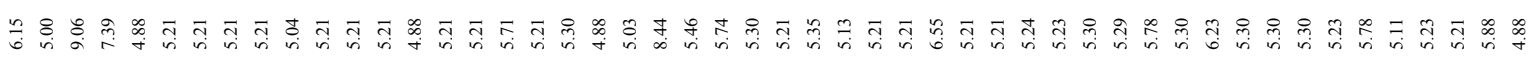

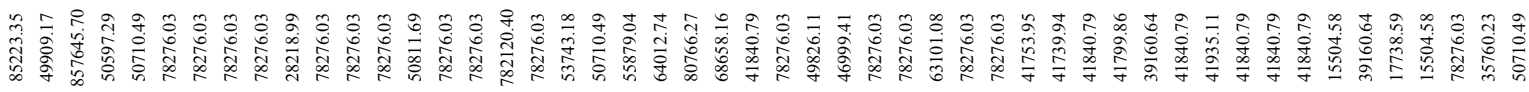

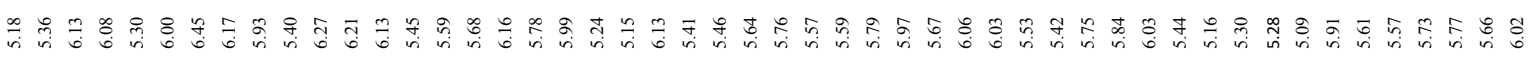

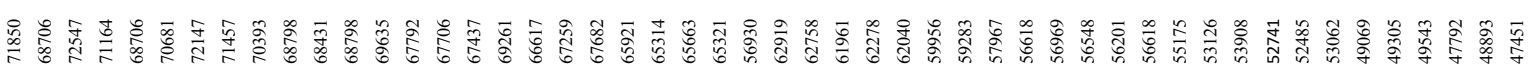

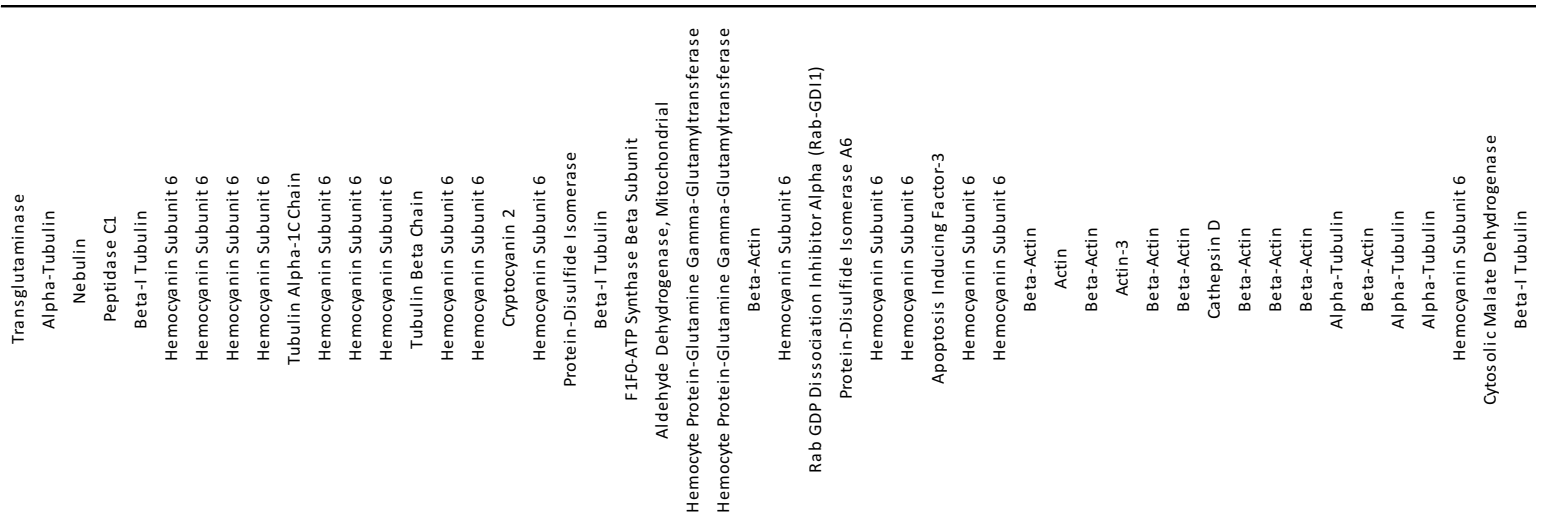

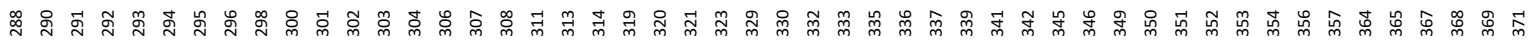



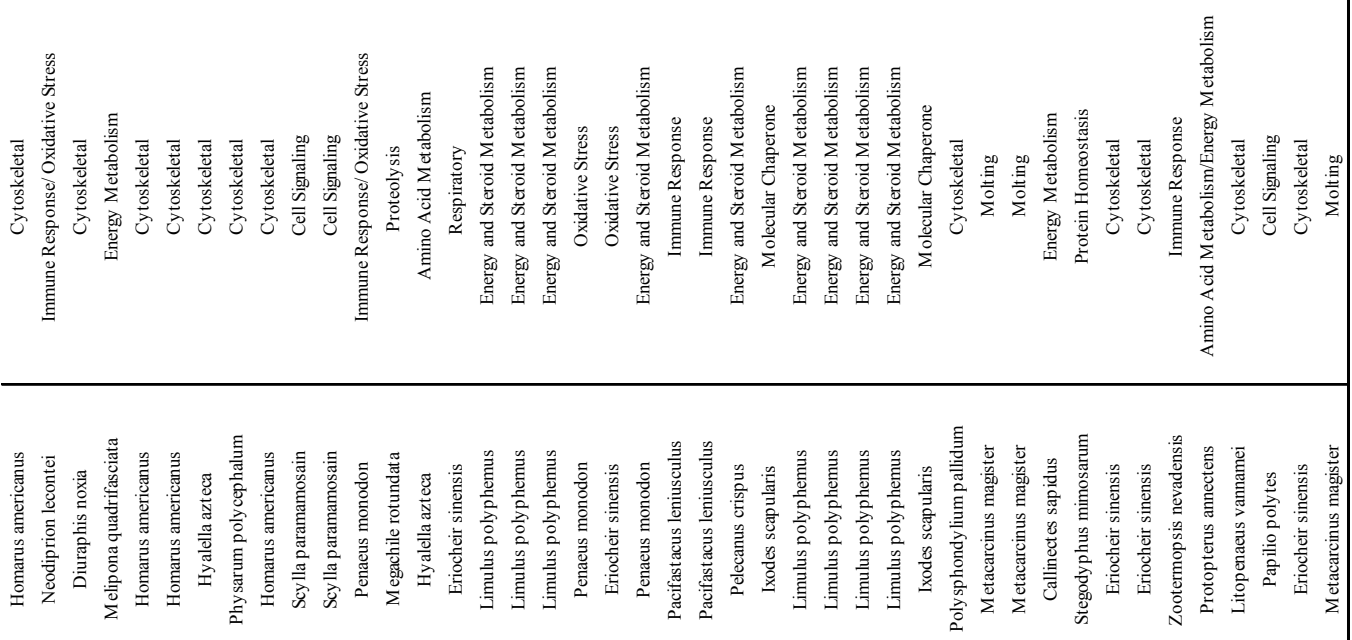

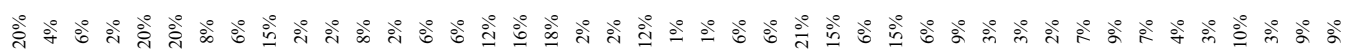

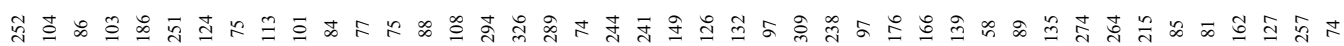

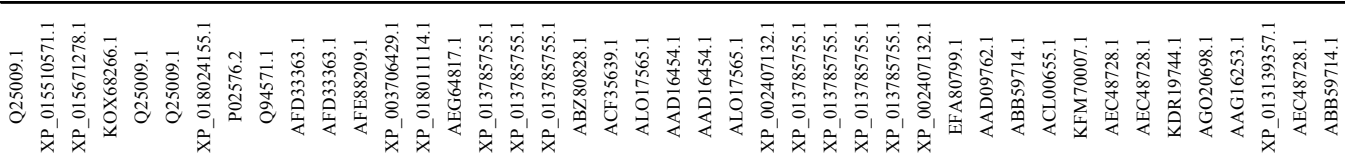

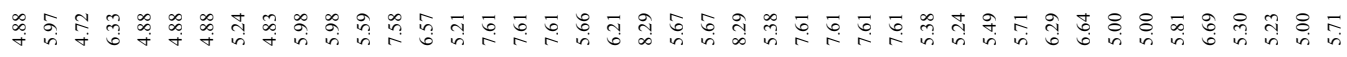
mmmmmmmmmmmmm

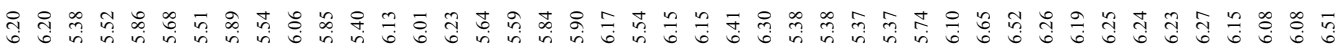

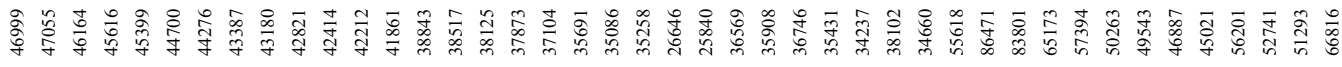

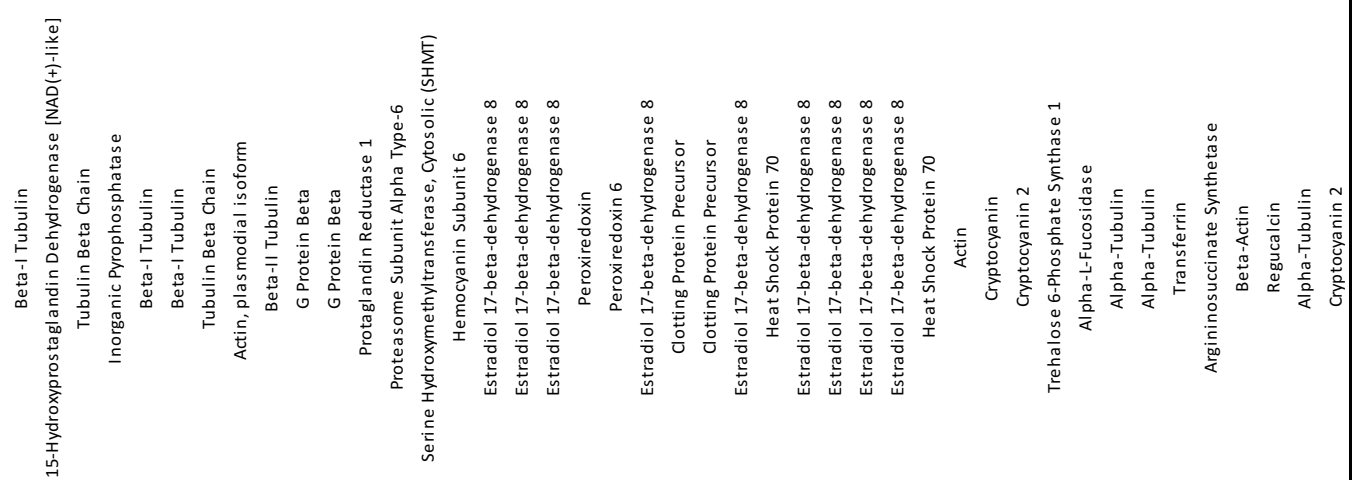

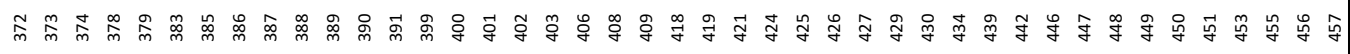




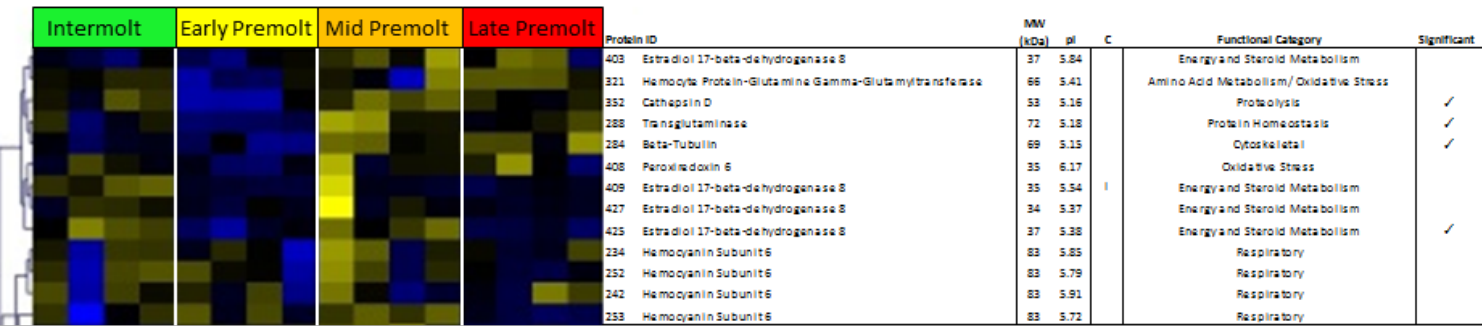

Abundance change:

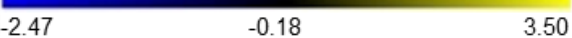

Figure 27A. Hierarchal clustering using Pearson's correlation depicting relative protein abundance (normalized spot volume) for Cluster I of all identified proteins observed within the Y-organ of Gecarcinus lateralis. Yellow boxes indicate a relative increase in protein abundance from the average, while blue boxes represent a lower than average protein abundance. Individual columns represent a single gel image composed of one biological replicate (3 pairs of Y-organs from animals in the same molting stage) for intermolt, early, mid, and late premolt stages as indicated above. Individual rows represent the standardized pattern of abundance for each identified protein. Five clusters are represented by separation between rows with a dashed line and represent groups of proteins which express similar changes in protein abundance among molt stages. Molecular weight (Da) and isoelectric point $(\mathrm{pI})$ for each protein were determined based on the location of each protein spot within the composite gel image using Decodon Delta 2D. 


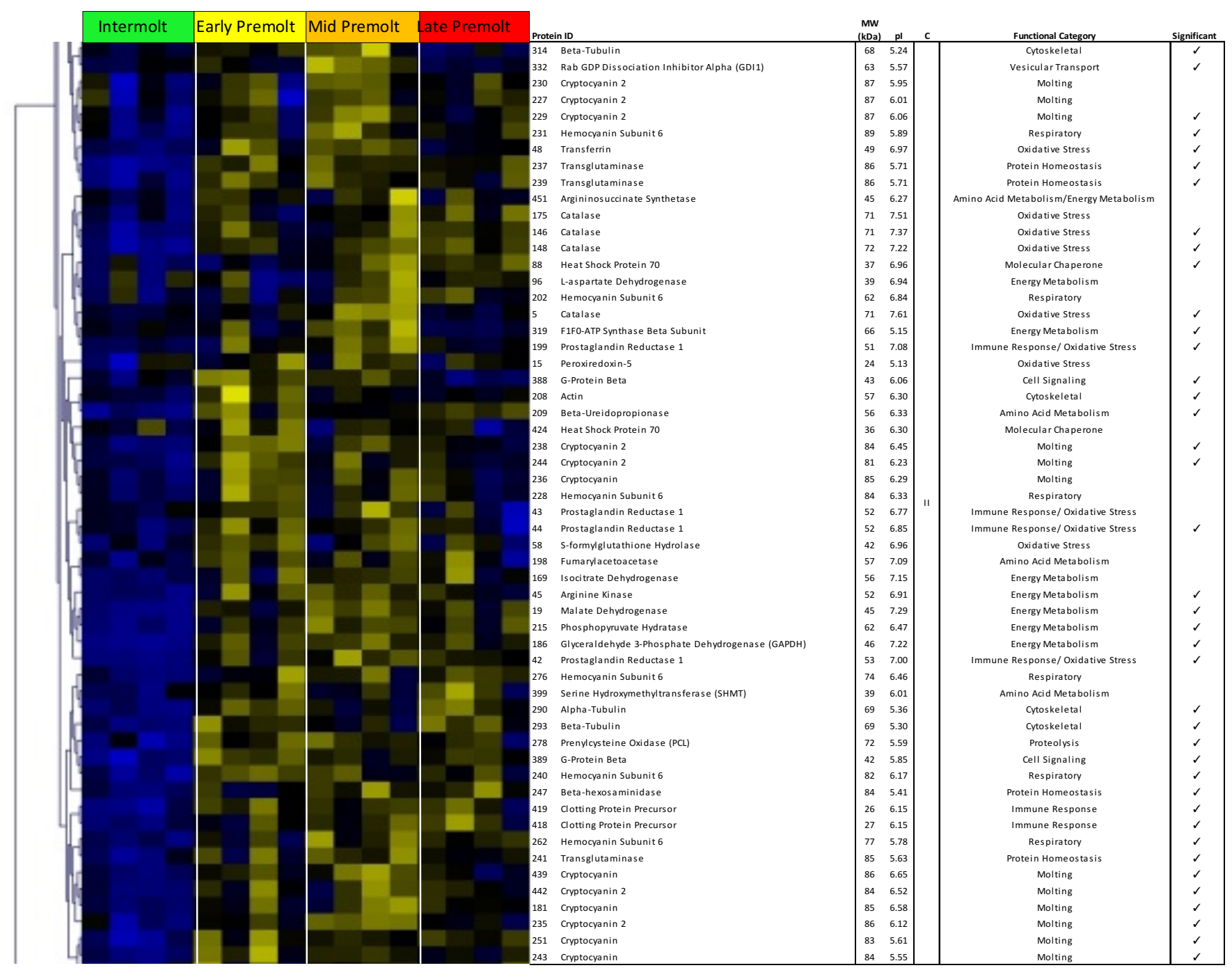

Abundance change:

$-2.47$

$-0.18$

3.50

Figure 27B. Hierarchal clustering using Pearson's correlation depicting relative protein abundance (normalized spot volume) for Cluster II of all identified proteins observed within the Y-organ of Gecarcinus lateralis. 


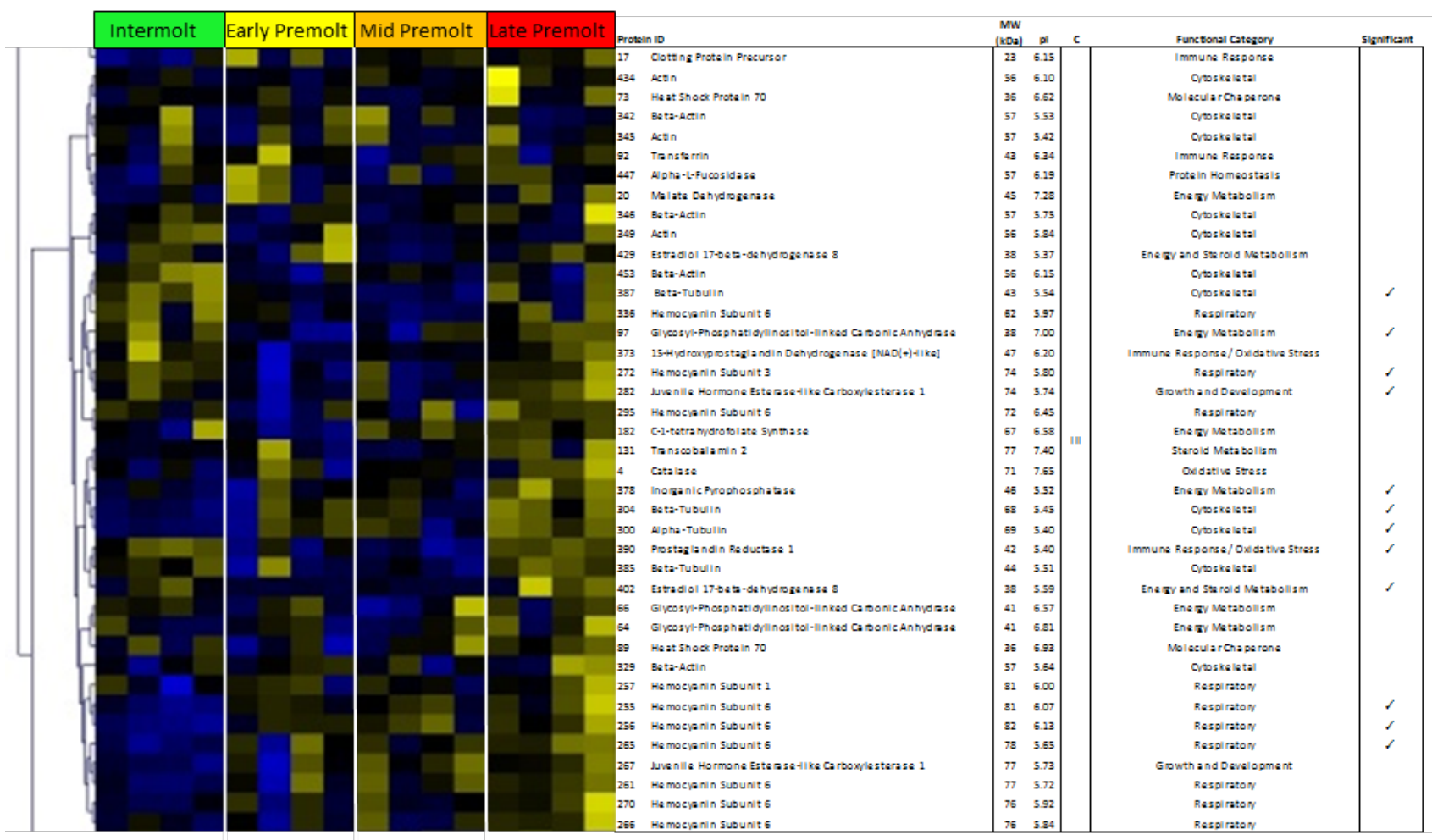

Abundance change.

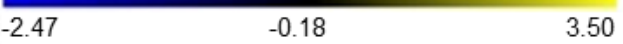

Figure 27C. Hierarchal clustering using Pearson's correlation depicting relative protein abundance (normalized spot volume) for Cluster III of all identified proteins observed within the Y-organ of Gecarcinus lateralis.

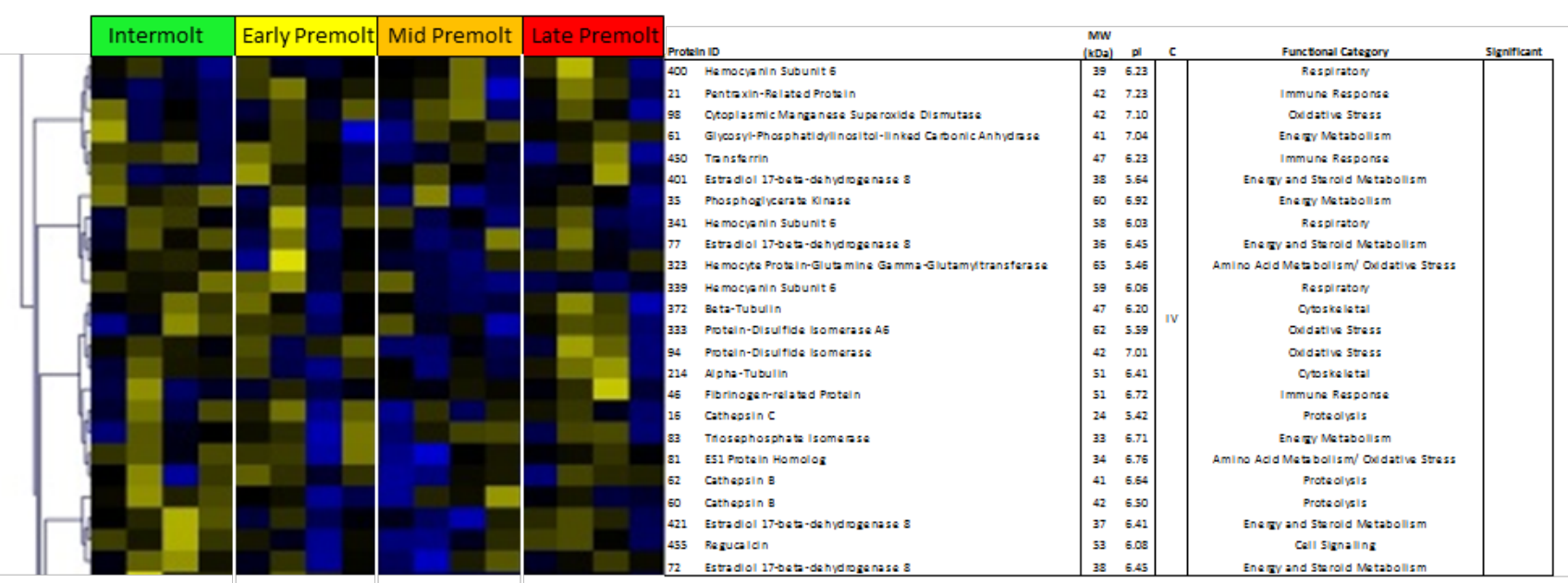

Abundance change

Figure 27D. Hierarchal clustering using Pearson's correlation depicting relative protein abundance (normalized spot volume) for Cluster IV of all identified proteins observed within the Y-organ of Gecarcinus lateralis. 

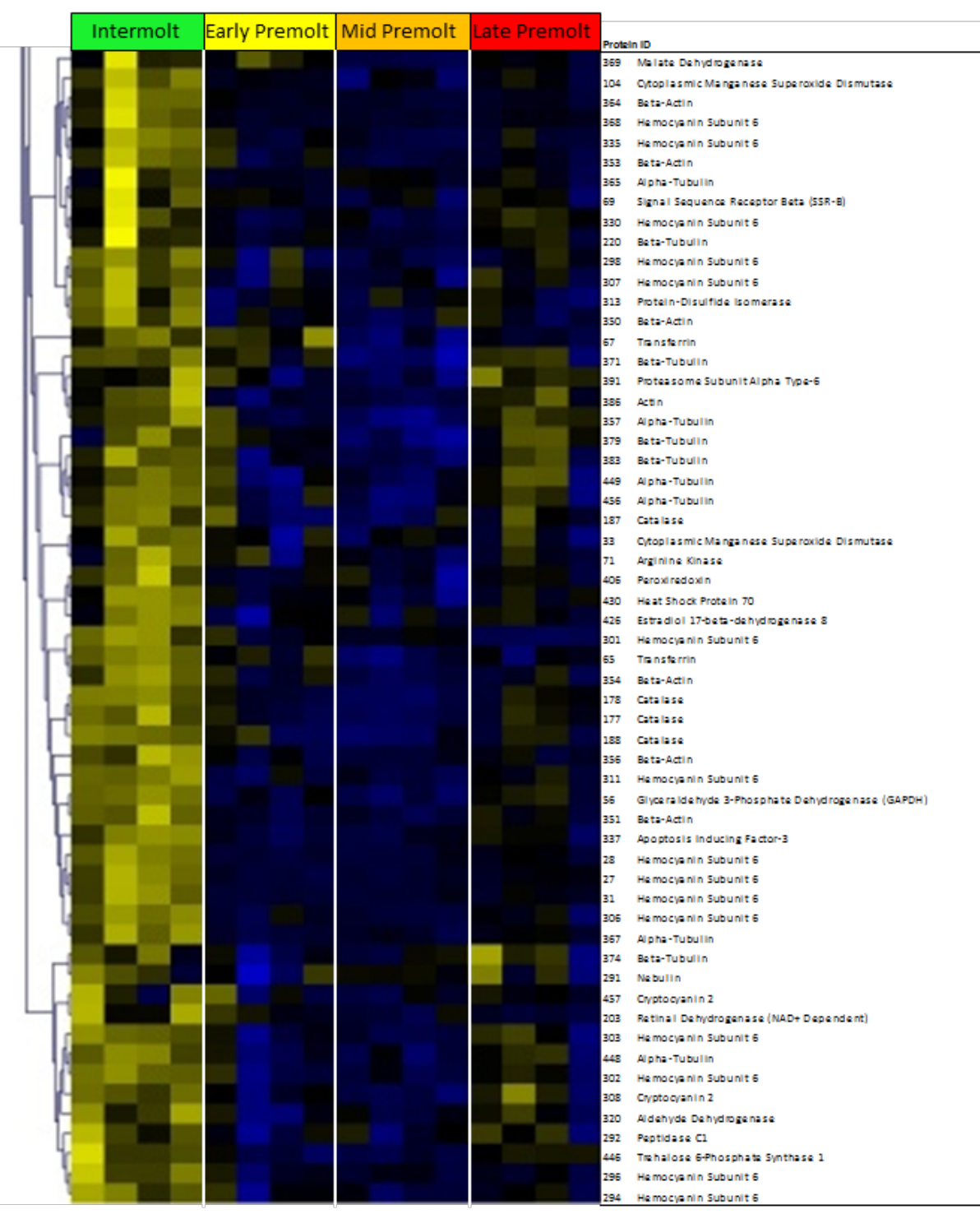

\begin{tabular}{|c|c|c|c|c|}
\hline$(1002)$ & pll & c & Functonal Catesory & Significant \\
\hline 495 & 5.56 & & Ene ary Metabollsm & 1 \\
\hline 39 & 7.08 & & Oedativa stass & $\checkmark$ \\
\hline 49 & 5.51 & & Croskeletal & 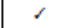 \\
\hline 48 & 5.77 & & Respiraton & 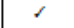 \\
\hline 62 & 5.79 & & Respiratory & r \\
\hline 54 & 530 & & Croskeletal & 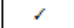 \\
\hline 49 & 3.57 & & Croskeletal & $r$ \\
\hline 39 & 6.88 & & Prota in Homsostasis & 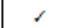 \\
\hline 63 & 5.75 & & Respiratory & 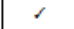 \\
\hline 48 & 6.45 & & Croskeletal & $\checkmark$ \\
\hline 70 & 5.93 & & Respiratory & 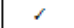 \\
\hline 67 & 5.68 & & Respiratony & $r$ \\
\hline 67 & 5.99 & & Oedative Stess & 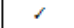 \\
\hline 57 & 6.03 & & Croskalatal & $\checkmark$ \\
\hline 39 & 6.35 & & Immune Responase & $r$ \\
\hline 47 & 6.02 & & Croskelstal & 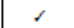 \\
\hline 42 & 6.13 & & Prota olyals & 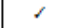 \\
\hline 43 & 5.99 & & Croskeletal & $\checkmark$ \\
\hline 53 & 3.91 & & Croskeletal & 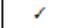 \\
\hline 45 & 5.55 & & Croskelatal & 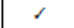 \\
\hline 45 & 5.68 & & Croskelatal & 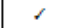 \\
\hline so & 6.24 & & Croskeletal & \\
\hline 51 & 6.08 & & Choskeletal & 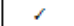 \\
\hline 54 & 7.43 & & Dedative Stess & \\
\hline 39 & 7.28 & & Oedative stess & 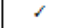 \\
\hline 38 & 6.54 & & Enery Metzbollsm & 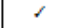 \\
\hline 36 & 5.90 & & Oedative Stress & 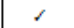 \\
\hline 35 & 5.74 & & Molscula a rona perone & 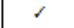 \\
\hline 35 & 5.38 & 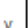 & Eneagy and Starold Matabollsm & 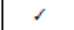 \\
\hline 68 & 6.27 & $v$ & Respiratory & 1 \\
\hline 41 & 6.35 & & Immune Responas & 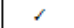 \\
\hline 53 & 5.28 & & Croskeletal & $r$ \\
\hline 61 & 7.44 & & Oxdative Stress & 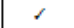 \\
\hline 61 & 7.47 & & Oedative Stress & 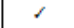 \\
\hline 34 & 732 & & Oedative Stress & 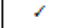 \\
\hline 52 & 5.09 & & Croskaletal & 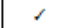 \\
\hline 57 & 5.78 & & Respiratory & 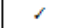 \\
\hline 43 & 6.55 & & Enear/ Matabollsm & 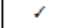 \\
\hline 55 & 5.44 & & Croskelstal & 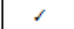 \\
\hline 60 & 5.57 & & Proga mmad cell death & $r$ \\
\hline 23 & 7.17 & & Respiratory & 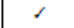 \\
\hline 24 & 7.45 & & Respiraton & 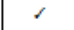 \\
\hline 24 & 7.65 & & Respiratony & $r$ \\
\hline 68 & 5.59 & & Respiratory & 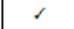 \\
\hline so & 5.73 & & Croskeletal & 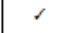 \\
\hline 45 & 5.38 & & Croskeletal & \\
\hline 73 & 6.13 & & Croskeletal & \\
\hline 67 & 6.51 & & Molting & \\
\hline 67 & 6.35 & & Dedative stress & $\checkmark$ \\
\hline 70 & 6.13 & & Respliraton & 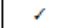 \\
\hline 50 & 6.25 & & Croskeletal & $r$ \\
\hline 69 & 6.21 & & Respiratory & 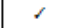 \\
\hline 69 & 6.15 & & Molting & 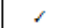 \\
\hline 65 & 6.13 & & Oedative Stess & 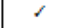 \\
\hline 71 & 6.08 & & Prota olyals & 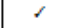 \\
\hline 65 & 6.25 & & Eneary Matzoollsm & 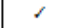 \\
\hline 71 & 6.17 & & Respiraton & 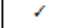 \\
\hline 71 & 6.00 & & Raspiratory & 1 \\
\hline
\end{tabular}

\section{Abundance change:}

$\begin{array}{lll}-2.47 & -0.18 & 3.50\end{array}$

Figure 27E. Hierarchal clustering using Pearson's correlation depicting relative protein abundance (normalized spot volume) for Cluster $\mathrm{V}$ of all identified proteins observed within the Y-organ of Gecarcinus lateralis. 


\begin{tabular}{|c|c|c|c|c|c|c|c|c|c|c|c|}
\hline Intermolt & Early Premolt & Mid Premolt & Late Premolt & Protel: & & $\begin{array}{l}\text { Mow } \\
(\mathrm{k} D \mathrm{a}\end{array}$ & pl & c & Functional Czasory & PC1 & PC2 \\
\hline & & & & $s 8$ & Hast Snock Prota in 70 & 37 & 696 & & Molacsuly Cheposaso & & \\
\hline & & & & 247 & Bata hexosa minidase & 34 & 241 & & Protsin Hompostas is & & \\
\hline & & & & 255 & Hamos/anin Subunit 6 & 81 & 607 & & Rapirzory & & \\
\hline & & & & 256 & Hamos/anin Subunit 6 & 82 & 6.13 & & Røpirzary & & \\
\hline & & & & 378 & Inorzanle Prophosphatase & 45 & 552 & & Enrag Matbolian & & 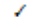 \\
\hline & & & & 304 & Bata-Tubullin & 68 & 145 & & Cyostik ob:al & & 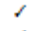 \\
\hline & & & & 300 & Alpha-Tubulin & 69 & 140 & & Cyostobeal & & $\checkmark$ \\
\hline & & & & 293 & Betz-Tubullin & 69 & 530 & & Cytodiabial & & r \\
\hline & & & & 290 & Alpha-Tubulin & 69 & 536 & & Cyrotiob:al & & s \\
\hline & & & & 209 & Bata-Ure idoproplonase & 56 & 633 & & Anino Adad Matabliam & & 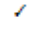 \\
\hline & & & & 278 & Pranyostaine Oxidase (PCL) & 72 & 559 & & Protsolyyis & & \\
\hline & & & & 399 & GProtain Bets & 42 & 285 & & Coll Signting & & 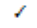 \\
\hline & & & & 240 & Hamos/anin Subunit 6 & $\$ 2$ & 617 & & Røpirzory & & \\
\hline & & & & 419 & Cotting Prosin in Praoursor & 26 & 615 & & Immyos Rospones & & \\
\hline & & & & 418 & Clotting Prosin Preoursor & 27 & 615 & & Immues Rosponses & & \\
\hline & & & & 146 & Catalase & 71 & 737 & & Oxistrivo Sress & 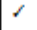 & \\
\hline & & & & 148 & catalase & 72 & 722 & & Oxidsivo Stress & 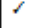 & \\
\hline & & & & 252 & Hamos/anin Subunit 6 & $n$ & 178 & & Rspirzory & & \\
\hline & & & & 241 & Transfivitaminase & ss & 263 & & Prosin Homoostas is & & \\
\hline & & & & 265 & Hamos/anin Subunit 6 & 78 & 265 & & Respirzory & & \\
\hline & & & & 251 & Crpasa nin & 83 & 161 & & Moling & & \\
\hline & & & & 243 & Crotoca nin & 84 & 555 & & Moling & & \\
\hline & & & & 237 & Transa futaminase & 86 & 171 & 1 & Protsin Hemroos tzis & ' & \\
\hline & & & & 239 & Transerutaminase & 86 & 271 & 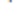 & Protsin Homoos tzis & 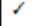 & \\
\hline & & & & 238 & Crvatocanin 2 & 84 & 645 & & Moling & & \\
\hline & & & & 208 & Aetin & 57 & 630 & & Cytothob:al & & 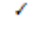 \\
\hline & & & & 244 & Crotocanin 2 & $\$ 1$ & 623 & & Moling & & \\
\hline & & & & 44 & Prostag: nd in Radutase 1 & 52 & 685 & & Immeo Rap onse Oxidsivo Stasus & & \\
\hline & & & & $38 s$ & Gerotain Bets & 43 & 606 & & Coll Sigating & & \\
\hline & & & & 314 & Batz-Tubulin & 68 & 524 & & Cytostob:al & & 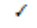 \\
\hline & & & & 332 & RaD GDP Dissociation Inhl biter Al pha (RaD-GDII) & 63 & 5257 & & Vovizoir Trampat & & ' \\
\hline & & & & 439 & Cnatoga nin & 86 & 665 & & Moling & & r \\
\hline & & & & 442 & Cratoga nin 2 & 34 & 652 & & Moling & & \\
\hline & & & & 131 & Cryatoganin & 3 & 20 & & Moling & & \\
\hline & & & & 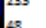 & Traberating 2 & 40 & 507 & & 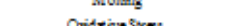 & 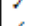 & 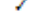 \\
\hline & & & & 229 & contoganing 2 & 37 & 606 & & Moling & . & 1 \\
\hline & & & & 231 & Hamos/anin Subunit 6 & 89 & 289 & & Repirzory & & 8 \\
\hline & & & & 5 & catalase & 71 & 261 & & Oxidsivo Sress & & 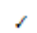 \\
\hline & & & & 319 & F1F0-ATP Symthase Beta & 66 & 215 & & Enory Matobliam & & 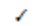 \\
\hline & & & & 199 & Prostay a ndin Raductase 1 & 51 & 208 & & Immuce Rap ones Oxidsivo Stans & & \\
\hline & & & & 45 & Ary nine Kinase & 52 & 691 & & Bnergy Mat boliam & & \\
\hline & & & & 19 & Malata Dehydropenase & 45 & 729 & & Enargy Matboliam & ' & \\
\hline & & & & 215 & Phosphopyulata Hidratase & 62 & 647 & & Bnary Matolinam & r & \\
\hline & & & & 185 & Gliceraldehyde 3-fhosphate Dahydrogenase (GAPDH) & 46 & 722 & & Energy Matbolinn & 8 & \\
\hline & & & & 42 & Prostay a ndin Raduetase 1 & 53 & 700 & & Immuce Rap ons Oxidstivo Stans & 1 & \\
\hline
\end{tabular}

Abundance change:

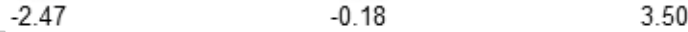

Figure 28A. Hierarchal clustering using Pearson's correlation depicting relative protein abundance (normalized spot volume) for Cluster I of proteins spots within the Y-organ of Gecarcinus lateralis which changed significantly in abundance with response to molt stage (One-way permutation ANOVA; $p<0.05)$. 


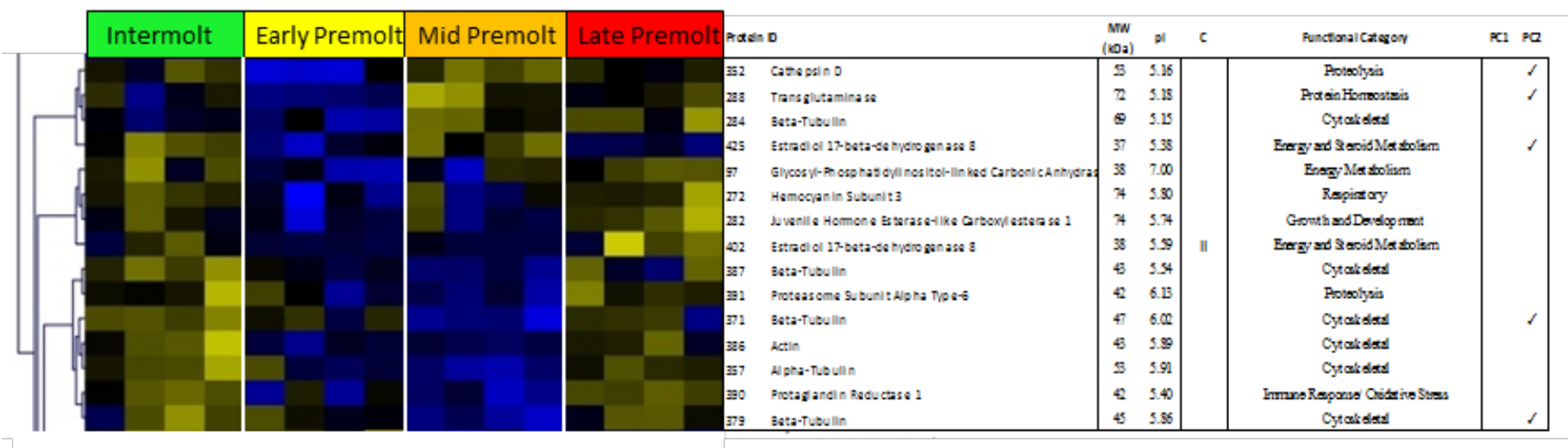

Abundance change:

Figure 28B. Hierarchal clustering using Pearson's correlation depicting relative protein abundance (normalized spot volume) for Cluster II of proteins spots within the Y-organ of Gecarcinus lateralis which changed significantly in abundance with response to molt stage (One-way permutation ANOVA; $p<0.05)$. 


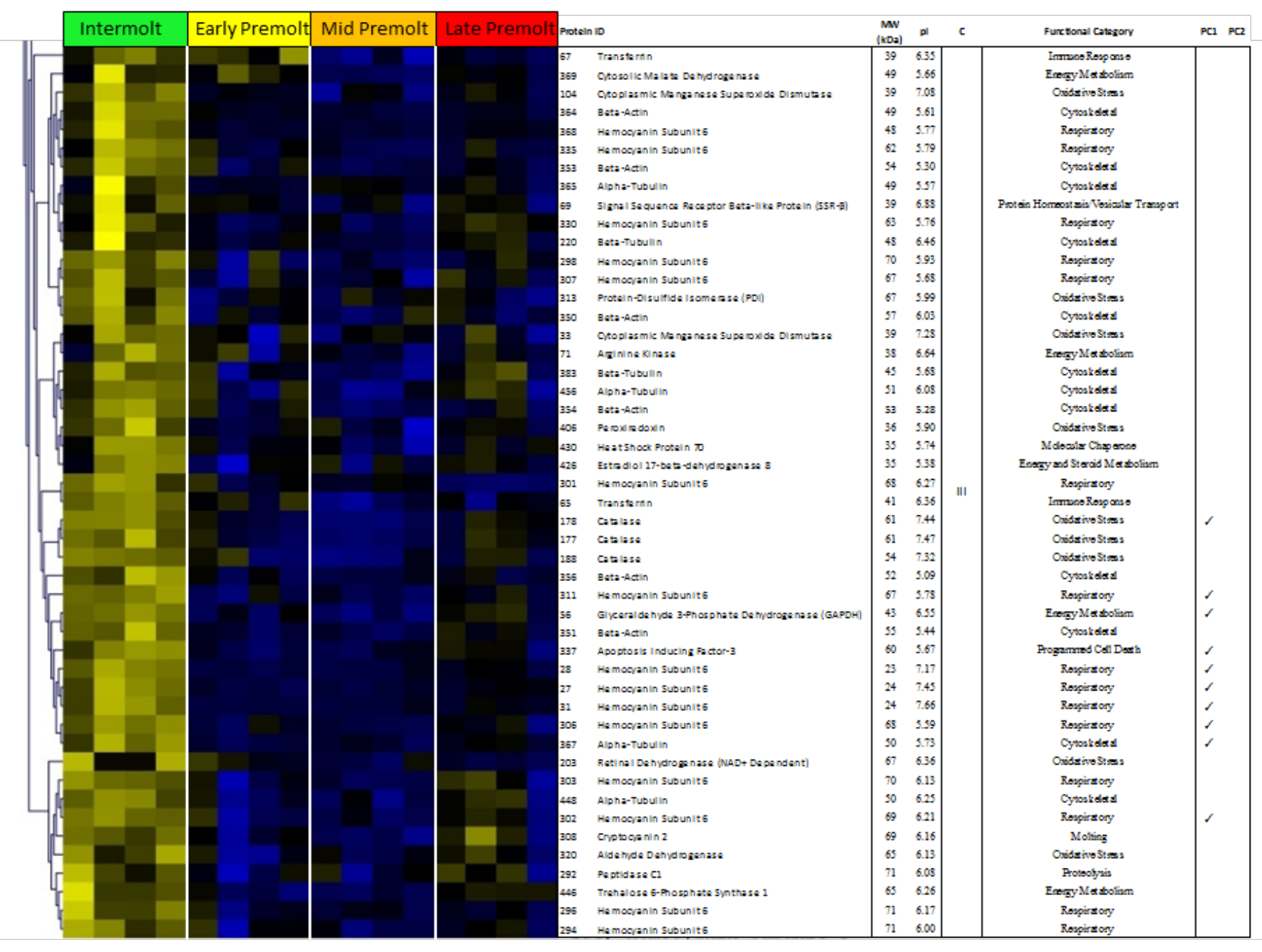

Abundance change:

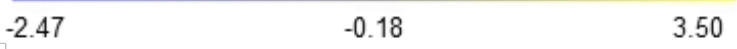

Figure 28C. Hierarchal clustering using Pearson's correlation depicting relative protein abundance (normalized spot volume) for Cluster III of proteins spots within the Y-organ of Gecarcinus lateralis which changed significantly in abundance with response to molt stage (One-way permutation ANOVA; $p<0.05)$. 